Andrews University

Digital Commons @ Andrews University

1972

\title{
The Relationship Between the Self-Concept and School Achievement in the Disadvantaged Child
}

Aneesi B. Nakhle

Andrews University

Follow this and additional works at: https://digitalcommons.andrews.edu/theses

Part of the Disability and Equity in Education Commons, and the Special Education Administration Commons

\section{Recommended Citation}

Nakhle, Aneesi B., "The Relationship Between the Self-Concept and School Achievement in the Disadvantaged Child" (1972). Master's Theses. 157.

https://dx.doi.org/10.32597/theses/157

https://digitalcommons.andrews.edu/theses/157

This Thesis is brought to you for free and open access by the Graduate Research at Digital Commons @ Andrews University. It has been accepted for inclusion in Master's Theses by an authorized administrator of Digital Commons @ Andrews University. For more information, please contact repository@andrews.edu. 


\title{
ABSTRACT OF GRADUATE STUDENT RESEARCH.
}

Thesis

\author{
Andrews University \\ Department of Education
}

Titie: The Relationship Between the Self-Concept and School Achievement in the Disadvantaged Child

Name of: researcher: Aneesi B. Nakhle

Name of faculty advisers: Dr. C. Reichert, Chairman; Dr. N. Burrell, and Dr. M. Youngberg

Date completed: August 17, 1972

PURPOSE

The purpose of the study was to determine the extent of the relationship between the self-concept and the academic achievement of the disadvantaged child.

\section{METHOD AND PROCEDURES}

The subjects studied were randomly selected from an inner city elementary school. The sample consisted of 97 black and 8 white children of ages 9 to 13, in a nongraded school. The pupils were administered the California Achievement Test Level 3 Form B, and the California Test of Personality, Level 2, Forti AA. Both tests were administered during the month of April 1972.

The statistical treatment of the data consisted of obtaining the coefficients of intercorrelation between the following four variables: 
(1) school achievement, (2) self-concept, (3) school placement level, (4) sex. The Pearson product-moment method of obtaining correlation coefficients was used, and a total of 720 coefficients were calculated by the use of a computer. In each case, the correlation between self-concept and school achievement was determined relative to school placement levels and sexes.

\section{RESULTS}

The data indicated that there was a positive and statistically significant correlation between the self-concept and academic achievement.

The results of the study are predictive and show that the lower the self-concept in the disadvantaged child, the lower will be his school achievement. Likewise the higher his self-concept, the higher will be his school achievement. 
Andrews University

School of Graduate Studies

THE RELATIONSHIP BETWEEN THE SELF-CONCEPT AND

SCHOOL ACHIEVEMENT IN THE DISADVANTAGED

CHILD

A Thesis

Presented in Partial Fulfillment

of the Requirements for the Degree

Master of Arts

by

Aneesi B. Nakhle

August 1972

Approva1 Cound Co Veidhet 
TABLE OF CONTENTS

Chapter

Page

I. INTRODUCTION . . . . . . . . . . . . . . . . . . 1

Purpose of the study . . . . . . . . . . . . . . 1

Hypotheses . . . . . . . . . . . . . . . 2

Organization of the study .............. . 2

II. REVIEW OF THE LITERATURE . . . . . . . . . . . . . . . 3

Definition of self-concept . . . . . . . . . . . 3

Development of the self-concept . . . . . . . . . . 4

Theories and research on self-concept . . . . . . . . 6

Summary .................... 12

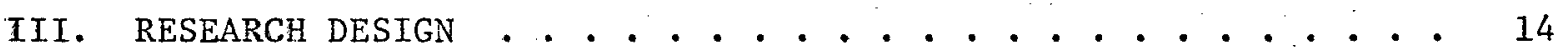

The population . . . . . . . . . . . . . . 14

Research instruments . . . . . . ....... 15

Research procedures . . . . . . . . . . . . 17

Statistical Design . . . . . . . . . . . 19

Definition of terms . . . . . . . . . . . . . 20

IV. ANALYSIS OF THE DATA •.................. . . 21

V. CONCLUSION AND IMPLICATION . . . . . . . . . . . . . 40

APPENDIX . . . . . . . . . . . . . . . . . . . . . 44

BIBLIOGRAPHY . . . . . . . . . . . . . . . . . . 48 


\section{LIST OF TABLES}

Table

Page

1. The Correlation Between Self-Concept Scores and School

Achievement Scores for All Subjects in All Levels . . . . 22

2. The Correlation Between Self-Concept Scores and Schoo1 Achievement Scores for Males . . . . . . . . . 24

3. The Correlation Between Self-Concept Scores and School Achievement Scores for Famales . . . . . . . . . 26

4. The Correlation Between Self-Concept Scores and School Achievement Scores for Level 4 . . . . . . . . . . 27

5. The Correlation Between Self-Concept Scores and School Achievement Scores for Level 5 . . . . . . . . . . 28

6. The Correlation Between Self-Concept Scores and School Achievement Scores for Level 6. . . . . . . . . . 29

7. The Correlation Between Self-Concept Scores and School Achievement Scores for Level 4, Males . . . . . . . . 31

8. The Correlation Between Self-Concept Scores and School Achievement Scores for Level 4, Females . . . . . . . . 32

9. The Correlation Between Self-Concept Scores and School Achievement Scores for Level 5, Males . . . . . . . . 33

10. The Correlation Between Self-Concept Scores and School Achievement Scores for Level 5, Females... . . . . . 34

11. The Correlation Between Self-Concept Scores and School Achievenent Scores for Level 6, Males . . . . . . . . . 35

12. The Correlation Between Self-Concept Scores and School. Achievement Scores for Level 6, Females . . . . . . . 37 
CHAPTER I

INTRODUCTION themselves.

That teacher helps his pupils most who most helps them to help --James Lawson

As a teacher, the writer has observed the effects of positive and negative self concepts in disadvantaged children. She has also observed the role that the educator of young children can play in the acquisition of adequate and accurate self-concepts. Symonds (1952, pp. 183-184) says:

Too often schools forget that their task is helping boys and girls grow up to be more efficient men and women. They become so immersed in the details of processes of growth that they forget the larger aims. Schools may become concerned with the development of such isolated skills as reading, writing, and arithmetic without providing that these skills be directed toward developing increased competence in affairs of life. The development of the ego may be considered the main task of education. Education should be concerned with helping individuals make better adjustments to their physical and social surroundings. Education should be concerned with more effective perceiving, more effective thinking, and more effective acting.

\section{PURPOSE OF THE STUDY}

Rogers, Combs, Snygg, Symonds, Jersild, and other educators and psycholgists recognize the relationship between the self-concept of children and their academic performance. It was Combs (1966) who said: "Any experience or situation which makes a person feel less liked, less wanted, less acceptable, less able, less dignified, less worthy--interferes 
with the process of learning."

The purpose of this study was to determine the extent of the relationship between the self-concept and academic achievement of the socially, culturally, economically, and educationally disadvantaged children.

\section{HYPOTHESES}

The hypotheses behind this study were as follows:

1. A positive correlation exists between the self-concept of the disadvantaged child and his school achievement.

2. There are no differences between disadvantaged males and females in the correlation between the self-concept and school achievement.

3. There are no differences between school placement levels in the correlation between the self-concept and school achievement in disadvantaged children.

\section{ORGANIZATION OF THE STUDY}

Chapter $I$ is organized to identify the purpose of this study.

Chapter II acquaints the reader with the 1iterature, the theory, and the research on the self-concept.

Chapter III describes the research design--the population, the tools, and the procedures that were utilized in this study.

Chapter IV discusses the findings and analysis of the data.

Chapter $\mathrm{V}$ focuses on the conclusions and implications. 
CHAPTER II

REVIEW OF THE LITERATURE

In the last several decades, a great deal of attention and interest has been focused upon the self-concept and its effects upon behavior. Early American psychologists, like William James, considered the self-concept important ir behavior.

In the early part of the twentieth century the self-concept theory fell into distepute as a result of the advent of Watson's behaviorism and Thorndike's connectionism and other attempts to qualify only observable acts in the analysis of buman behavior. As Gestalt psychology, however, began to spread in the United States the self-concept again came to be considered crucial in understanding behavior. Under the leadership of such famous psychologists as Car1 Rogers, Donald Snygg, Arthur Combs, and Abraham Maslow, this Gestalt-field theory movement has culminated today in a body of theory known as phenomenology. In phenomonology, the self is the central variable in behavior as well as in education and learning.

\section{Definition of Self-Concept}

The term in Freudian psychology that is closest to self-concept is the construct of the ego. Adlerian psychology refers to it as the life style. Fromm used the term, self realization. Combs and Snygg talk about the phenomenal self. Kelly explains the fully functioning self. Other terms such as Maslow's actualizing self; Rogers' congruent self; 
Jersild's "inner world"; and Bugental's authentic self are also used.

The self-concept has been defined as those perceptions, beliefs, feelings, attitudes and values one accepts as descriptive of onself-implying that self-concepts are based upon one's perception of others' responses to him; as well as his own perceptions of his characteristics and abilities.

Combs and Snygg (1.959, p. 127) define it as "the symbol or generalization of self which aids in perceiving and dealing with self." They further point out that the self-concept is the central core of one's personality structure. Change in the individual's behavior can only occur in relationship to events which are perceived as having a direct bearing on seif.

Dr. Woolner (1971, p. 60) explores the dynamics of how we see ourselves. "Three major components, self-concepts, ideal self-concept, and other self-concept, should be understood as a whole. Let's call this whole the global self-concept. It consists of the traits, attributes, and limitations the individual assigns to himself (his self-concept); the individual's evaluation of himself (his ideal self-concept); and how the individual perceives what others think of him (his other self-concept).

\section{Development of the Se1f-Concept}

The self-concept develops slowly over the years, generalization coming about as a result of repeated environmental reinforcements. Once developed, though incompletely, change does occur all through life but it occurs slowly.

In the early years, the relations of the parents are most important, 
and, because the mother tends to spend more time with the child than does the father, her personality and behavior are of crucial importance during these years. When the child is fondled and petted; when his parents quickly respond to his needs, and he is given evidence by their smiles and delight that he is loved and desired, his perceived self reflects feelings of security and self-esteen. If he is ignored, nagged, and fails to please he will feel insecure and inadequate. On the whole, a child's level of self-regard is associated with his parents' reported regard for him (Wylie, 1961).

When the child enters school, other influences on self evaluation intervene. His role within the family may have been the cause for acceptance within the home but it has little influence at school. At school he must demonstrate his worth. Attitudes toward parents and cthers may be displaced to teachers. Concerning self-concept as it pertains to a school situation, Arthur T. Jersild (1952, pp. 10-19) said:

The self is a complicated subjective system which the learner brings with him to school. There is continuous impact between the self and the flow of experiences involved in the process of learning and living at school.

As the child matures toward pre-adolescence and adolescence, the reinforcement from peer groups may influence him more than reinforcement from parents or other adults. Kelley (1962, p. 19), sums up the development of the self-concept by stating that: "When we see that the psychological self is built mostly out of the people around it, we can see that the day never comes when the individual can continue to thrive without people." 
Theories and Research on Self-Concept

The importance of the self-concept in human behavior is demonstrated by Rogers (1951, p. 40) who found that psychotherapy deals primarily with the organization and functioning of the self. Lecky (1951, p. 153) sees all of an individual's behavior as organized into a single system, the nucleus of which is the individual's valuation of himself.

The individual is seen by Stephenson (1953, p. 244) as not being able to behave independently of the way he thinks of himself. The individual also rejects, experience that is inconsistent with his self-concept. (Rogers, 1951, p. 236). Consequent1y, what a person thinks of himself, consciously and unconsciously, is one of the prime determiners of his behavior.

Jersild (1952) commented that when a person resists learning that may be beneficial to him, he is, in effect trying to protect or shield an unhealthy condition.

In a study by Page (1958), pupil performance improved to a statistically significant degree when teachers wrote encouraging comments on pupils' papers. A control group given a conventional mark without comments lost ground in performance during the same time.

Based on a study made by Brookover and Lapere (1962), a research project was conducted with parents of low-achieving ninth-grade students. As a result of treatment for one semester, some parents changed their evaluations of their children's abilities to do better in school, and some students were so affected by the change that a gain of a grade-point average was shown by 42 percent of them. This percent gain was raised significantly by the end of the year. 
Sylvester and Kunet (1963), while investigating why the child does not see himself as a potential learner, developed the theory of the inhibition of exploratory function. They reported the results of differential treatments of thirteen children, aged 8 to 13 , who were under treatment for reading disabilities. In one case tutoring oniy was used; in another psychotherapy; in the remaining eleven cases both tutoring and psychotherapy were utilized. They postulated that teaching alone was not enough--where tutoring alone succeeded it was because the tutor intuitively met some of the emotional needs of the child.

Addressing the Michigan Department of Education staff in May, 1966, Dr. Arthur Combs in his lecture "Modern Concepts of Learning" stated that: "Those who succeed in school subjects do so largely because of their positive self-concept. Those who fail do so largely because of their negative self-concept."

Bledsoe (1967, pp. 436-438), conducted a study to find the relationship between the self-concepts of fourth and sixth grade boys and girls in relation to their intelligence, academic achievement, interests and manifest anxiety. He chose a random sample of 271 fourth and sixthgrade boys and girls from four schools in Clark County, Georgia. The self-concept scores were obtained by administering a Self-Concept Scale consisting of a checklist of 30 trait-descriptive adjectives which the subject checked as characteristic of himself "nearly always," "about half the time," or "fust now and then." The results of his testings are given below:

Means and standard deviations for the four groups of puplis on self-concept (SC)were as follows: 


\begin{tabular}{|c|c|c|c|c|}
\hline \multicolumn{3}{|c|}{ BOYS } & \multicolumn{2}{|c|}{ GIRLS } \\
\hline & 4 th & 6th & $\begin{array}{c}4 \text { th } \\
\text { Grde }\end{array}$ & $\begin{array}{c}6 \text { th } \\
\text { Grade }\end{array}$ \\
\hline Mean & 73.1 & 73.9 & 78.3 & 78.4 \\
\hline S.D. & 10.1 & 8.0 & 7.4 & 6.5 \\
\hline N. & 65 & 76 & 60 & 70 \\
\hline
\end{tabular}

There was no appreciable difference between the SC of boys in the two grades, or of the girls in the two grades. But the girls in both grades scored significantly higher. (at the .01 level) than the boys in the corresponding grades.

Correlations between SC and intelligence for the four groups were mostly low to moderately positive. For boys, the correlations. were all significant and positive, ranging from .28 to .41 . For girls, correlations were non-significant.

Similarly, correlations of achievement and SC for boys were significant and positive; for girls they were non-significant. The mean $x$ (using the $z$ transformation) for fourth-grade boys between SC and achievement was . 38 for fourth-grade boys and . 35 for sixthgrade boys.

Although all except one of the fourteen $r^{\prime} s$ between $S C$ and achievement for girls were non-significant, the direction was positive. The one significant $r$ was .29 between $S C$ and reading comprehension for fourth-grade girls.

Manifest anxiety and SC correlated negatively as follows: fourth boys, -.35 ; sixth boys, -.46 ; fourth girls, -.30 ; sixth girls, -.22 . Al1 r's except that of sixth girls were significant. Thus in general self-esteem were associated with lower expressed anxiety.

Culturally disadvantaged children, according to much of the research (Witty, 1967, p. 384), seem to mirror the negative attitudes of others and reflect this discrimination in their own negative self-images.

Anthony T. Soares and Louise M. Soares (1969, pp. 31-45), conducted a study which was concerned with the self-concept of disadvantaged children in the middle and upper grades of elementary school. They studied 514 subjects in an urban school system with 229 subjects coming from a public elementary school in a disadvantaged area, and 285 subjects coming from a public elementary school in an advantaged area. In their 
fina1 analysis the authors noted that both the disadvantaged and advantaged children indicated positive self perceptions, which were neither overly high nor unduly low. Thus they concluded that despite their cultural handicap, disadvantaged children do not necessarily suffer from lower self esteem and lower sense of personal worth. Also they note that ( $p$. 44) "It may well be that the common denominator is effective and realistic teaching."

Fink (1962, pp. 57-62) found a significant relationship between adequacy of self-concept and level of academic achievement. Piers and Harris (1964, pp. 91-95) found that the relationship between self-concept scores and I.Q., as well as academic achievement was greater at the sixth grade than at the third grade level.

The role of self-concept in achievement was also studied by Billy J. Pascha11 (1968, pp. 392-396) of Ball State University. The purpose of his investigation was to compare two groups of Ss whose scores on the Spivack Response Form led to classification of adequate self-concept or inadequate self-concept. The subjects were randomly selected from the seventh grade class of a junior high school in Dale County, Florida. Academic achievement was determined by over all grade-point-average. Those whose GPA fell at or above a " $\mathrm{C}$ ". average were considered achievers, and those whose GPA fell below a "C" average were considered nonachievers. In his conclusion $\mathrm{Dr}$. Paschall points out that the results indicate that a relationship does exist between reported self-concept and teacher assigned grades.

Morris D. Caplin (1968, pp. 435-439) in his study of the selfconcept, level of aspiration and academic achievement, concluded the 
Fo1lowing:

(p. 437) Significant differences were indicated among schools on the self-concept and the level of aspiration analycis.

(pp. 437,438) On the basis of statistical findings in this study it may be concluded that there were no significant differences between children attending the de facto segregated, the long-term desegregated, or the newly desegregated schools when that part of the self-concept and level of aspiration related to personal social qualities was considered.

(p. 438) However, it was found that the school-related self-concept and level of aspiration of children, white and Negro, attending the de facto segregated school were significantly lower than those of children attending the newly-desegregated or long-term desegregated schools. It also was found that children having more positive selfconcepts and high levels of aspiration had higher academic achievement.

It is important to note that the subjects in this study were representative of the lowex socio-economic class. The locality in which the study was made is a small city, located near New York City. The aity had a Negro population of about 20 percent concentrated in one relatively small area.

Dr. Romeo Eldridge Phillips of Kalamazoo College (1969, pp. 32-37) studied the relationship between student activities and self-coricepts. High findings indicate that participation in the activity program at Marquis High School (a suburban high school outside Detroit with an eighty percent Negro student population), is significantly related to self-concepts for boys, but not for girls nor for the total sample." No significant relationship was found for girls between participation in any activity and scores on a self-concept measure. A significant relationship was found between participation in athletics and self-concept scores for first-string male athletes, but not for second-string athletes.

Fred L. Pigge (1970, pp. 107-114) reports on three studies that 
were conducted on children and their self-concepts. The first one was: "Group Counseling and the Altering of Self-Concepts" by Bill F. Payne and Charleta J. Dunn. Regarding this study Pigge (1970, p. 108) commented:

Resurts of the analysis indicate that group counseling altered favorably the self-concepts of the participants in the investigation on referents "Mother," "Peer," "Self and Subjects," "Self as Object" and the total referent score. The experimental group made a higher mean difference score (significant at the .05 level) on pre-post-tests than did the control group only on referent "Mother." Mean scores on referent "Teacher" were most resistant to change. An explicit implication of the data is that the classroom lends itself to promoting positive self-concepts if students are utilized as change-agents.

The second study reported by Pigge (1970) was conducted by Zadie B. Whisenton on "The Effects of Planned Experiences with Outstanding Negro Children." The results of this study have shown that Negro children who interact with Negro adult models of achievement may thereby enhance their attitudes toward self. Positive attitudiral changes may occur in other differentiations of their perceptual fields, but less rapidly.

The third study was entitled "Relationships Between Self-Concepts and Reading Achievement at the Third-Grade Level" by Ruby Nell Cummings. Pigge (1970) in his research report mentioned that the purpose of the study was to investigate similarities and differences existing in the reported self-concepts of certain third-grade children when their scores were grouped according to criteria based on their reading achievement. The specific problem was to find whether there were differences in the reported self-concepts of 4 groups of children. A sumary of the findings is as follows (Pigge, 1970, p. 114):

The referents that seem most important in the school situation, "Teacher". and "Peers,". were the ones that inadequate readers scored lowest in relation to their total self-concept. Ability, the value factor important for academic success in the school classroom, was 
scored lowest by those children who felt themselves inadequate according to their reported self-concepts.

Positive self-concepts were found to be related to adequate reading achievement. The most significant differences were found between groups based on reading level in their own classroom. Children's reading achievement in comparison to that of others in their own classroom seemed more related to their self-concepts than achievement in comparison to one's own anticipated reading achievement.

"Self-Concepts and Ethnic Group Membership Anong Pubiic School Students" is the title of a study that was conducted by Perry A. Zirkel and E. Gnanaraj Moses (1971, pp. 253-265). The study indicated that ethnic group membership in a public school significantly affects the selfconcepts of individual students. This effect, however, seems to be attributable to the presence but not to the minority-majority proportion of the ethnic group within the school.

Project SEED (Special Elementary Education for the Disadvantaged), a 45-minutes Mathematics program that is conducted four times a week, is designed to improve the self-concepts of culturally, socially, and economically disadvantaged children. The program is built on the theory that a child's self image is a strong and vital factor in determining what he will become later in life. The present researcher has observed this program in action in the Benton Harbor Area Schools District, Michigan, in which she was a teacher during the past two years. It is her impression that many of the discipline problems were eliminated and more student participation was evident. No statistical findings, however, were published to show any results of this project.

\section{Summary}

The foregoing chapter has reviewed the literature on the self- 
concept, defined it and discussed its role in deternining behavior. It also told how the self-concept is developed and explored its theories and research.

It has been shown that the self-concept develops from what an individual learns to think about himself by observing what others think of him; and that the individual's seif-concept is never completely developed, but rather keeps forming and reforming throughout life.

There has been an increasing number of empirical studies on selfconcept since 1949, revealing a "bewildering array" of hypotheses, instruments, operational definitions, and research designs. Researchers have studied the relationship of self-concept to such variables as achievement, socioeconomic status, I.Q., sex, anxiety, level of aspiration, student activities, interests, reading, group counseling, planned experiences with outstanding Negro citizens, and ethnic group membership. The results of these investigations vary from one study to another; some show significant relations, others indicate a positive trend, but few results have been consistent from one study to another.

However significant, positive, or inconsistent the findings have been, it is evident that self-concept is an important part of the psychological make-up and the scholastic performance of the individual. 
CHAPTER III

\section{RESEARCH DESIGN}

\section{The Population}

The sample for this study was selected from Martin Luther King $\mathrm{Jr}$. Elementary School in Benton Harbor, Michigan. Martin Luther King Jr. Elementary School is one of seven schools in the Benton Harbor Area Schools District that follow the IGE program.

IGE (Individually Guided Education), is one of the new concepts in current educational trends. It encourages a multi-level (grade) classroom. "Level" Four is a school placement level which includes, for instance, pupils of ages nine and ten regardless of the grade they have completed in school. The students are pre-tested at the beginning of each year and at the beginning of each unit in study. They are then posttested at the close of each year and at the completion of each unit of study. Upon the results of these tests the children are individually guided to work with materials that they can compete with and which will give them success. Thus, they are encouraged to keep climbing the ladder of learning by succeeding a step at a time.

Martin Luther King Jr. Elementary School is an intermediate school with Levels Four, Five, and Six. The total school population at the time of the present study was 403 students. The school is considered to be a Target School, also, with children who are socially, culturally, economically, and educationally disadvantaged. 
From the total school population a sample of 105 subjects was randomly selected by the use of a table of random numbers (Fisher and Yates, 1963). The results of the selections were as follows:

$\begin{array}{ll}\text { Leve1 } 4 & \begin{array}{l}23 \text { Males } \\ 16 \text { Females }\end{array} \\ \text { Level } 5 & 16 \text { Males } \\ & 15 \text { Females } \\ \text { Leve1 } 6 & 18 \text { Males } \\ & 17 \text { Females }\end{array}$

of the 105 subjects in the sample, 97 were black and 8 were white since the majority of the population is black.

\section{Research Instruments}

The study was designed to find the correlation between self-concept and achievement, and two types of tests were employed to obtain the necessary data.

A. The California Achievement Test, 1970 Edition, Leve1 3 Form B (Appendix A ). This battery spans grades 4 through 6 . The CAT, 1970 Edition was designed for the measurement, evaluation, and analysis of school achievement. The emphasis is upon content and objectives in the basic curricular areas of reading, mathematics, and language.

B. The California Test of Personality, Level 2, Form AA (Appendix $B$ ). This is a self report inventory available in five levels. It spans the age range from Kindergarten to college students and adults, and yields fifteen scores. The following descriptions define twelve separate aspects of self-concept measured by the instrument: (Thorpe, Clark, and Tiegs, 1953, pp. 3,4) 


\section{PERSONAL ADJUSTMENT}

1A. SELF-RELIANCE--An individual may be said to be self-reliant when his overt actions indicate that he can do things independently of others, depend upon himself in various situations, and direct his own activities. The self-reliant person is also characteristically stable emotionally, and responsible in his behavior.

1B. SENSE OF PERSONAL WORTH--An individual possesses a sense of being worthy when he feels he is well regarded by others, when he feels that others have faith in his futire success, and when he believes that he has average or better than average ability. To feel worthy means to feel capable and reasonably attractive.

1C. SENSE OF PERSONAL FREEDOM--An individual enjoys a sense of freedom when he is permitted to have a reasonable share in the determination of his conduct and in setting the general policies that shall govern his life. Desirable freedom includes permission to choose one's own friends and to have at least a little spending money.

1D. FEELING OF BELONGING--An individual feels that he belongs when he enjoys the love of his family, the well-wishes of good friends, and a cordial relationship with people in general. Such a person will as a rule get along well with his teachers or employers and usually feels proud of his school or place of business.

1E. WITHDRAWING TENDENCIES--The individual who is said to withdraw is the one who substitutes the joys of a fantasy world for actual successes in real life. Such a person is characteristically sensitive, lonely, and given to self-concern. Normal adjustment is characterized by reasonable freedom from these tendencies.

1F. NERVOUS SYMPTOMS--The individual who is classified as having nervous symptoms is the one who suffers from one or more of a variety of physical symptoms such as loss of appetite, frequent eye strain, inability to sleep, or a tendency to be chronically tired. People of this kind may be exhibiting physical expressions of emotional conflicts.

\section{SOCTAL ADJUSTMENT}

2A. SOCIAL STANDARDS--The individual who recognizes desirable social standards is the one who has come to understand the rights of others and who appreciates the necessity of subordinating certain desires to the needs of the group. Such an individual understands what is regarded as being right or wrong.

2B. SOCIAL SKILLS--An individual may be said to be socially skillful or effective when he shows a liking for people, when he inconveniences himself to be of assistance to them, and when he is. 
diplomatic in his dealings with both friends and strangers. The socially skillful person subordinates his or her egotistic tendencies in favor of interest in the problem and activities of his associates.

2C. ANTI-SOCIAL TENDENCIES--An individual would normally be regarded as anti-social when he is given to bullying, frequent quarreling, disobedience, and destructiveness to property. The antisocial person is the one who endeavors to get his satisfactions in ways that are damaging and unfair to others. Normal adjustment is characterized by reasonable freedom from these tendencies.

2D. FAMILY RELATIONS--The individual who exhibits desirable family relationships is the one who feels that he is loved and welltreated at home, and who has a sense of security and self respect in connection with the various members of his family. Superior family relations also include parental control that is neither too strict nor too lerient.

2E. SCHOOL RELATIONS--The student who is satisfactorily adjusted to his school is the one who feels that his teachers like him, who enjoys being with other students, and who finds the school work adapted to his level of interest and maturity. Good school relations involve the feeling on the part of the student that he counts for something in the life of the institution.

2F. COMMUNTTY RELATIONS--The individual who may be said to be making good adjustments in his community is the one who mingles happily with his neighbors, who takes pride in community improvements, and who is tolerant in dealing with both strangers and foreigners. Satisfactory commity relations include as well the disposition to be respectful of laws and of regulations pertaining to the general welfare.

\section{Research Procedures}

The Population: To obtain the sample for this study the writer obtained a student list from each teacher in the school and assigned a number to each student. Using a table of random numbers (Fisher and Yates, 1963) a total number of 120 subjects was selected. However, because of the absenteeism of some on the days of testings, and due to the moving away of others, the total number of students that completed both tests was 105 . 
The California Achievement Test: The administration of this test was conducted by the individual classroom teachers during the last two weeks in April, 1972. Each teacher was responsible for his/her home-room since this was part of the school's testing program. The present researcher administered the test in her home-room only. The results of these tests were made avallable in duplicate to the researcher through the kindness of Dr. W. Saygh, Director of Testing and Assessment, Benton Harbor Area Schools District.

The California Test of Personality: Since this test was not part of the testing program, the researcher was financially responsible for obtaining the test booklets, the answer sheets, and the scoring of these tests. The tests were purchased from the McGraw-Hi11 Inc., Monterey, California.

Arrangements were made, with Mrs. Greta Hines, principal of the school, to administer this test during the second week in April 1972. For efficiency purposes the sample was divided into four groups. The testing was scheduled as follows:

$\begin{array}{lll}\text { Group A } & \text { April 10,1972 } & 1: 00-2: 00 \mathrm{p} \cdot \mathrm{m} . \\ \text { Group B } & \text { Apri1 11, 1972 } & 12: 00-1: 00 \mathrm{p} \cdot \mathrm{m} . \\ \text { Group C } & \text { April 12,1972 } & 12: 00-1: 00 \mathrm{p} \cdot \mathrm{m} . \\ \text { Group D } & \text { Apri1 13,1972 } & 1: 00-2: 00 \mathrm{p} \cdot \mathrm{m} .\end{array}$

A test booklet, an answer sheet, and a pencil with an erasure were made available to each student. After presenting the test and giving all the necessary directions, the researcher instructed the subjects to follow as she read each question, and to mark their answers on the answer 
sheet. The tests were read to the subjects because it was felt that

there might be a great variation in the reading ability of the subjects.

\section{Statistical Design}

Variables: The following variables were examined in the analysis of the realtionship between school achievement and the self-concept:

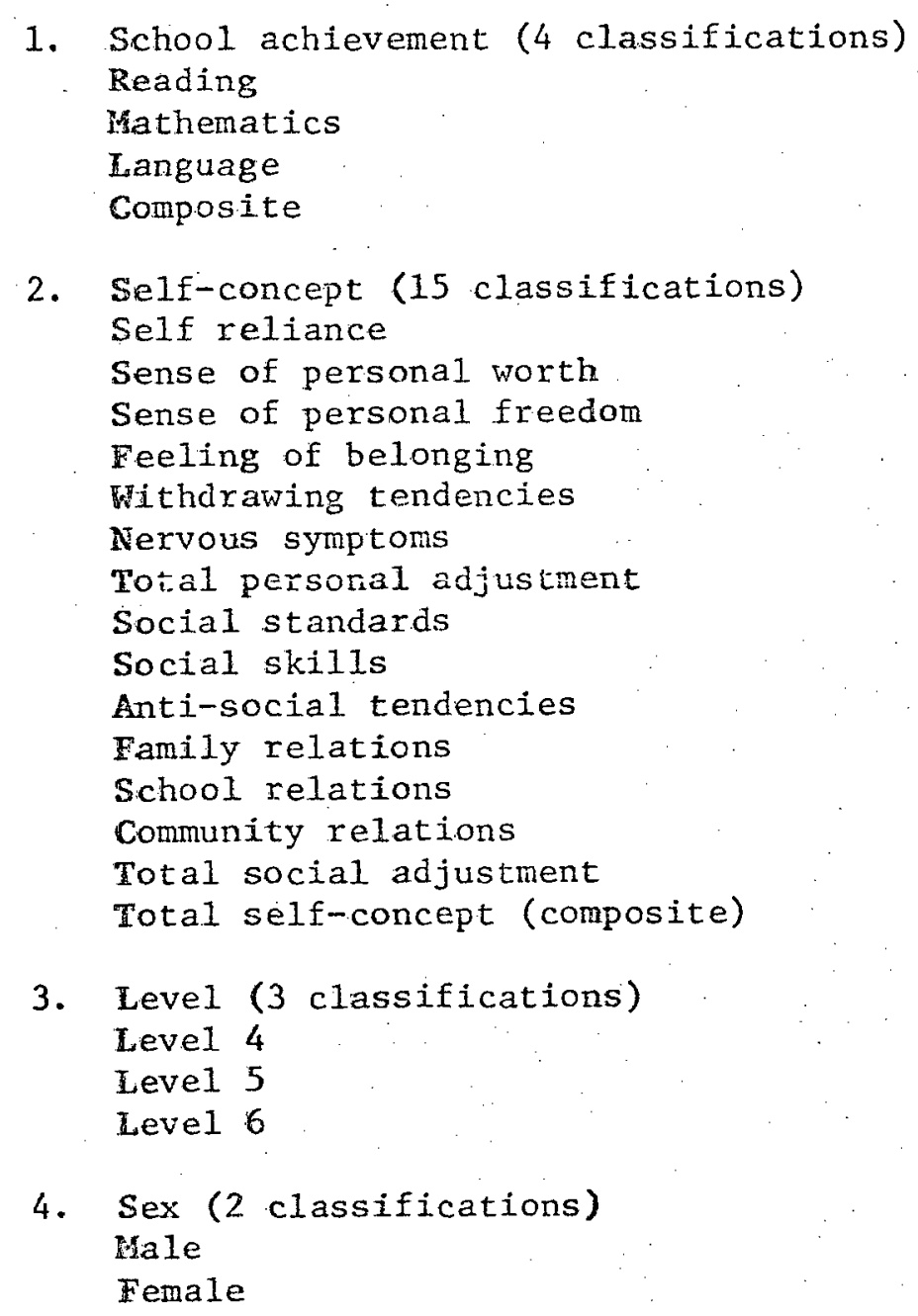

Statistical Design: The statistical treatment of the data consisted of obtaining the coefficients of inter-correlations between the four variables listed in the previous section. The Pearson product moment method of obtaining correlation coefficients was used, and a total of 720 coefficients were calculated by the use of a computer. 
In each case, the correlations between school achievement and self-concept were determined within school placement levels and sexes.

\section{Definition of Terms}

Socially, culturally, and economically disadvantaged: The expression "socially, culturally, and economically disadvantaged" in this study refers to those students who come from homes that have the characteristics of the lower-lower class. According to Warner (1960), the lowerlower class has the typical characteristics of depending on public assistance, such as welfare, for its source of income. Their homes are sma11, and they have large families.

Target Schools: Dr. Millie Urbish (1970, p. 12) defines the target school as follows:

Target schools in the Benton Harbor Public. School System are schools where a minimum of $25 \%$ of the pupils come from homes which receive Aid to Dependent Children Funds, are on welfare, or come from low income homes earning below $\$ 3000$.

\section{Educationally disadvantaged (deprived): Are those students who}

score below the 15 th percentile on their achievement tests. According to the principal of Martin Luther King Jr. Elementary School over 260 of the school's 403 pupils scored below the 15 th percentile.

This study involved a sample of 105 students. The majority of the population at the school is black, and the random selection of the sample produced 97 black and 8 white subjects. The population of Martin Luther King Jr. Elementary School is, therefore, socially, culturally, economically, and educationally disadvantaged according to the above mentioned definitions. 
CHAPTER IV

ANALYSIS OF THE DATA

In this stidy academic achievement is represented by the grade equivalent scores obtained on the California Achievement Test. This instrument yields four scores. Three of these scores represent achievement in reading, mathematics, and language. The fourth score represents the composite achievement across the three academic areas.

The California Test of Personality was used to determine the students' self-concepts. This test is divided into two major divisions: personal adjustment, and social adjustment. Under each of these two divisions are six sections containing twelve questions per section. The answer to each question consists of either a "yes" or "no" (Appendix B). Scores for each section were computed on the basis of correct responses (relative to well adjusted persons) on the twelve items in each section. Each of the two major divisions has a possible score of 72 , with a possible composite score of 144 for the entire test. A high score indicates "good" adjustment, and a low score indicates "poor" adjustment.

The Pearson product-moment method of obtaining correlation coefficients was used for obtaining coefficients of intercorrelations between the four variables listed in the previous chapter. A total of 720 coefficients were calculated by the use of a computer.

Analys is of the data for the overall sample: Table 1 indicates that there is a positive and significant correlation $(r=.28, p<.01)$ 
TABLE 2

THE CORRELATION BETWEEN SELF-CONCEPT SCORES AND SCHOOL ACHIEVEMENT FOR ALL SUBJECTS IN ALL LEVELS

\begin{tabular}{|c|c|c|c|c|c|c|c|c|c|c|c|c|c|c|c|}
\hline$\cdots$ & \multicolumn{7}{|c|}{ PERSONAL ADJUSTMENT } & \multicolumn{7}{|c|}{ SOCIAL ADJUSTMENT. } & \multirow[b]{2}{*}{ 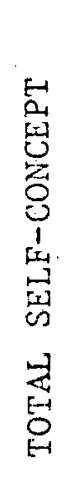 } \\
\hline $\begin{array}{c}\text { SCHOOL } \\
\text { ACHIEVEMENT }\end{array}$ & 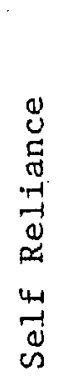 & 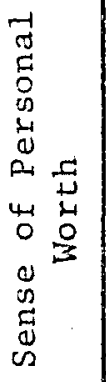 & 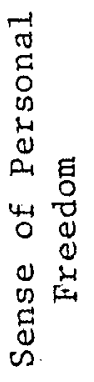 & 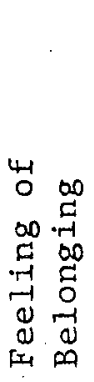 & 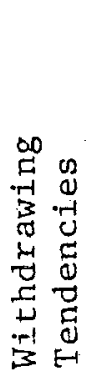 & 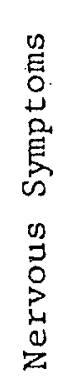 & 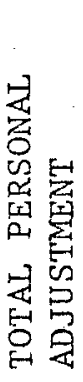 & 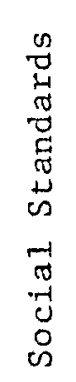 & 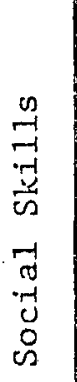 & 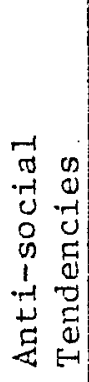 & 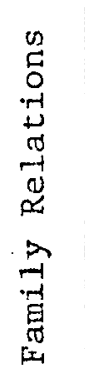 & $\begin{array}{c}n \\
E \\
0 \\
0-1 \\
+1 \\
0 \\
-1 \\
0 \\
04 \\
-1 \\
0 \\
0 \\
0 \\
0 \\
0 \\
0\end{array}$ & 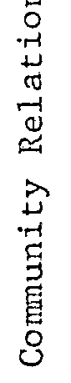 & 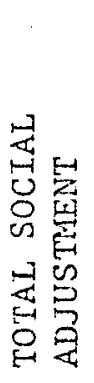 & \\
\hline & & $*$ & & $x * x$ & $\star$ & & $x *$ & $*$ & & & & & & & $\dot{x} * \dot{*}$ \\
\hline Reading : & .03 & .22 & .08 & .25 & .20 & .09 & .23 & .20 & .19 & .09 & .06 & .08 & .18 & .18 & .25 \\
\hline Math & .02 & .15 & .24 & .26 & .10 & .12 & .24 & .14 & .16 & .06 & .07 & .04 & .14 & .15 & .22 \\
\hline & & & $* * *$ & $* * 5$ & & & $*$ & $* * *$ & $2 *$ & & & & $* *$ & $* \dot{*} *$ & $x * x$ \\
\hline Language & .05 & .18 & .30 & .23 & $\therefore 10$ & .03 & .22 & .30 & .24 & .13 & .14 & .12 & .24 & .26 & .29 \\
\hline & & $\dot{x}$ & $* *$ & $* * *$ & & & $x * 2$ & $x *$ & $*$ & & & & $*$ & $\star$ & $* x *$ \\
\hline TOTAL BATTERY & .03 & .20 & .24 & .27 & .13 & .08 & .25 & .24 & .21 & .11 & .11 & .09 & .21 & .22 & .28 \\
\hline
\end{tabular}

$$
\begin{array}{rl}
* P & <.05 \\
* & P<.02 \\
* * * P & <.01
\end{array}
$$$$
N=105
$$ 
between self-concept (total battery) and school achievement (total battery).

Table 1 further indicates a number of positive and significant correlations such as: between total self-concept and reading achievement $(r=.25, p<.01)$, between total self-concept and mathematics achievement $(r=.22, p<.05)$, and between total self-concept and language achievement $(\mathrm{r}=.29, \mathrm{p}<.01)$. It also shows a positive and significant correlation between total personal adjustment and reading achievement $(r=.23, p<.02)$, between total personal adjustment and mathematics achievement $(r=.24, p<.02)$, between total personal adjustment and language achievement $(\mathrm{r}=.22, \mathrm{p}<.05)$, and between total personal adjustment and total achievement battery $(r=.25, p<.01)$.

Of the three school achievement areas--reading, mathematics, and language--Table 1 indicates that language achievement is more highly correlated with social adjustment than the other two achievement areas. By examining the table we find the following: $r=.30(p<.01)$ between social standards and language achievement; $r=.24(\mathrm{p}<.02)$ between social skills and language achievement; $\mathrm{r}=.24(\mathrm{p}<.02)$ between community relations and language achievement; and $r=.26(p<.01)$ between total social adjustment and language achievement.

Although these coefficients are not large, they possess statistical significance and point out a definite correlation between the self-concept and academic achievement.

Analysis of the data for males: Table 2 indicates that there is " positive and significant correlation $(r=.29, p<.05)$ between self-. 
TABLE 2

THE CORRELATION BETWEEN SELF-CONCEPT SCORES AND SCHOOL ACHIEVEMENT

MALES

\begin{tabular}{|c|c|c|c|c|c|c|c|c|c|c|c|c|c|c|c|}
\hline & \multicolumn{7}{|c|}{ PERSONAI ADJUSTMENT } & \multicolumn{7}{|c|}{ SOCIAL ADJUSTMENT } & \\
\hline $\begin{array}{c}\text { SCHOOL } \\
\text { ACHIEVEMENT }\end{array}$ & 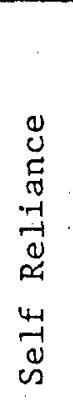 & 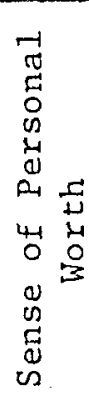 & 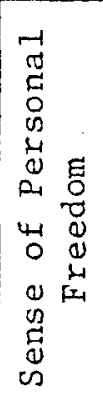 & 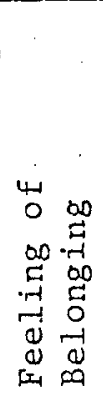 & 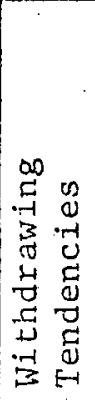 & 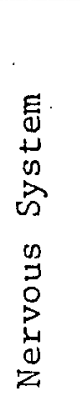 & 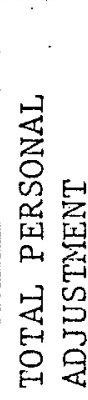 & 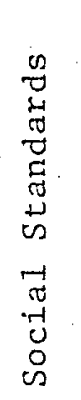 & 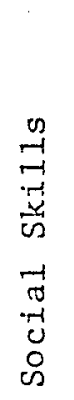 & 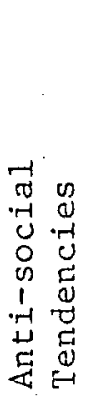 & 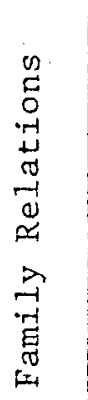 & 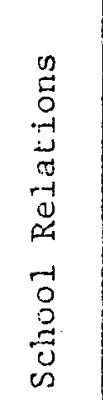 & 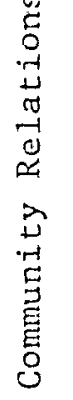 & 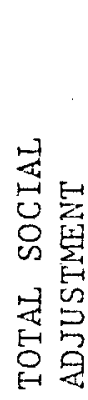 & 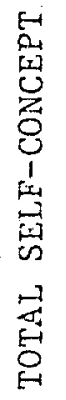 \\
\hline Reading & .11 & .09 & -.03 & .22 & .14 & .01 & .13 & .18 & .08 & .05 & .08 & -.05 & .09 & .13 & .15 \\
\hline Math & .13 & .21 & .19 & .32 & .09 & .04 & .22 & .25 & .13 & .15 & .11 & .06 & .10 & .19 & .25 \\
\hline Language & .16 & .11 & .21 & .22 & .08 & .01 & .18 & .33 & .15 & .10 & .17 & .12 & .12 & .23 & .25 \\
\hline TOTAL BATTERY & .06 & .09 & .20 & .26 & .14 & .06 & .21 & .30 & .19 & .24 & .11 & .15 & .18 & .28 & .29 \\
\hline
\end{tabular}

$\mathrm{N}=57$

$* P<.05$

$* * \mathrm{P}<.02$ 
concept (total battery) and school achievement (total battery) when looking at all males in all levels (Levels 4,5 , and 6).

Analysis of the data for females: Although Table 3 does not indicate for females a statistically significant correlation between total self-concept and total school achievement, there were significant correlations between nervous symptoms and reading achievement, between social skills and language achievement, and between cormunity relations and language achievement.

Analysis of the data across the three levels: Level 4 (males and females): Table 4 does not show a statistically significant correlation between total self-concept and total school achievement, however it indicates a positive and significant correlation between total selfconcept and language achievement $(r=.32, \mathrm{p}<.05)$.

Level 5 (males and females): Table 5 does not indicate a statistically significant correlation between total self-concept and total school achievement. However, reading stands out as an achievement area which indicates a positive and statistically significant correlation with total self-concept $(x=.36, p<.05)$.

Level 6 (males and females): Unlike Tables 4 and 5, Table 6 indicates that there is a statistically significant correlation between total self-concept and total school achievement $(r=.38, p<.05)$.

Levels 4 and 6 both indicated a positive and statistically significant correlation between total self-concept and language achievement. 
TABLE 3

THE CORRELATION BETWEEN SELF-CONCEPT SCORES AND SCHOOL ACHIEVEMENT SCORES

FEMALES

\begin{tabular}{|c|c|c|c|c|c|c|c|c|c|c|c|c|c|c|c|}
\hline & \multicolumn{7}{|c|}{ PERSONAL ADJUSTMENT } & \multicolumn{7}{|c|}{ SOCIAL ADJUSTMENT } & \\
\hline $\begin{array}{l}\text { SCHOOL } \\
\text { ACHLEVEMENT }\end{array}$ & 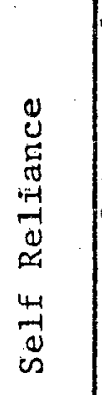 & 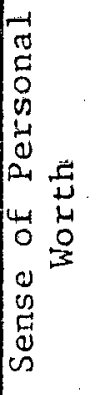 & 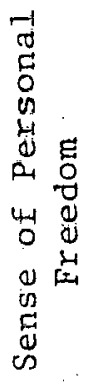 & 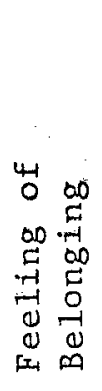 & 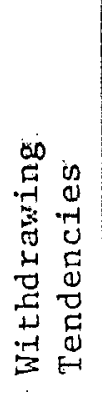 & 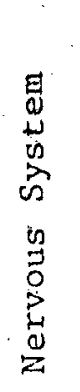 & 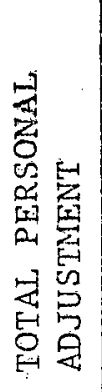 & 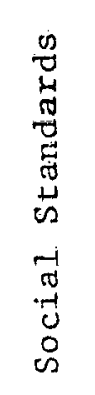 & 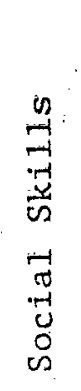 & 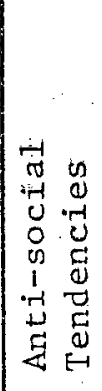 & 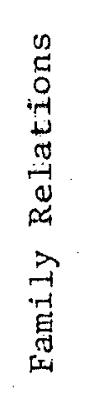 & 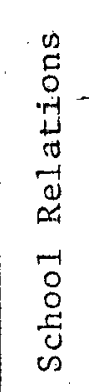 & 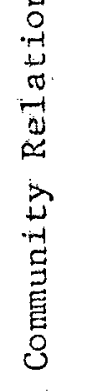 & 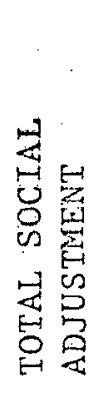 & 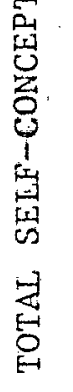 \\
\hline Reading & -.02 & .21 & .07 & .21 & .27 & $.30^{*}$ & $\begin{array}{r}* \\
.29\end{array}$ & .13 & .19 & .08 & .05 & .11 & .20 & .17 & .26 \\
\hline Math & -.08 & .05 & .27 & .18 & .12 & .27 & .22 & -.05 & .15 & -.07 & -.04 & -.04 & .16 & .02 & .14 \\
\hline Language & .04 & .02 & .28 & .22 & .25 & .11 & .24 & .12 & .29 & .24 & .04 & .11 & .34 & .28 & .28 \\
\hline TOTAL BATTERY & -.04 & .12 & .22 & .21 & .20 & .27 & .26 & .07 & .19 & .05 & -.02 & .01 & .24 & .13 & .23 \\
\hline
\end{tabular}

$N=48$

$* \mathrm{P}<.05$

$* * \mathrm{P}<.02$ 
TABLE 4

THE CORRELATION BETWEEN SELF-CONCEPT SCORES AND SCHOOL ACHIEVEMENT SCORES LEVEL 4

\begin{tabular}{|c|c|c|c|c|c|c|c|c|c|c|c|c|c|c|c|}
\hline \multirow[b]{2}{*}{$\begin{array}{c}\text { SCHOOL } \\
\text { ACHIEVEMENT }\end{array}$} & \multicolumn{7}{|c|}{ PERSONAL ADJUSTMENT } & \multicolumn{7}{|c|}{ SOCIAL ADJUSTMENT } & \\
\hline & 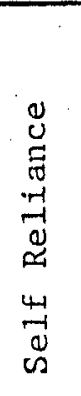 & 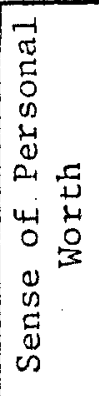 & 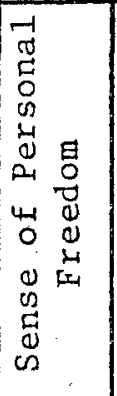 & 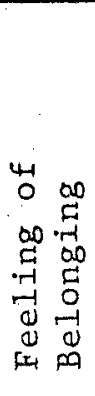 & 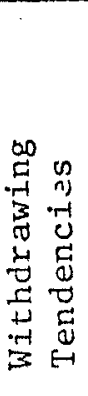 & 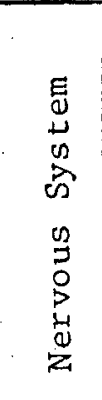 & 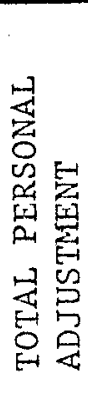 & 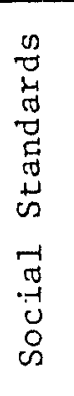 & 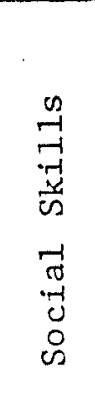 & 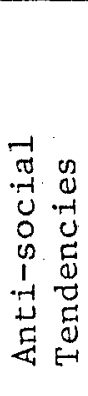 & 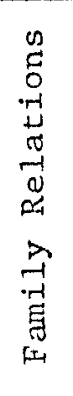 & 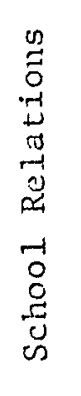 & 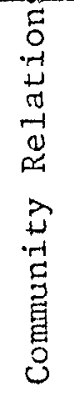 & 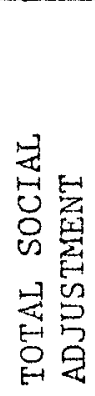 & 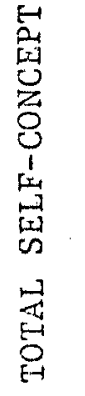 \\
\hline Reading & .21 & .22 & .01 & .26 & .22 & -.07 & .19 & $\begin{array}{l}* \pi x * \\
.41\end{array}$ & -.06 & .19 & .23 & .15 & $\begin{array}{l}x * \\
.39\end{array}$ & $\begin{array}{r}* \\
.35\end{array}$ & .31 \\
\hline Math & .18 & .25 & .14 & .27 & .09 & .03 & .22 & .17 & -.06 & .06 & .16 & .18 & .24 & $\begin{array}{r}.17 \\
*\end{array}$ & $\begin{array}{r}.23 \\
*\end{array}$ \\
\hline TOTAL BATTERY & .19 & .27 & .14 & .22 & .14 & -.00 & .24 & $\begin{array}{r}.50 \\
* \% \\
.39\end{array}$ & -.09 & .24 & .21 & .14 & $\begin{array}{r}.28 \\
x \\
.34\end{array}$ & .32 & .32 \\
\hline
\end{tabular}

$N=39$

$* \mathrm{p}<.05$

$* * \mathrm{P}<.02$

$x * x \quad \mathrm{P}<.01$ 
TABLE 5

THE CORRELATION EETWEEN SELF-CONCEPT SCORES AND SCHOOL ACHIEVEMENT SCORES LEVEL 5

\begin{tabular}{|c|c|c|c|c|c|c|c|c|c|c|c|c|c|c|c|}
\hline \multirow{2}{*}{$\begin{array}{c}\text { SCHOOL } \\
\text { ACHIEVEMENT }\end{array}$} & \multicolumn{7}{|c|}{ PERSONAL ADJUSTMENT } & \multicolumn{7}{|c|}{ SOCIAL ADJUSTMENT } & \multirow[b]{2}{*}{ 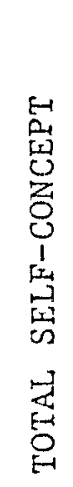 } \\
\hline & 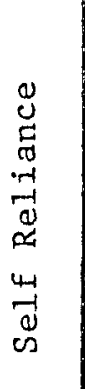 & 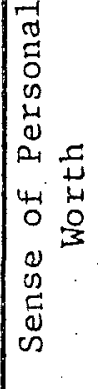 & 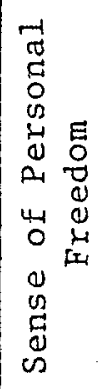 & 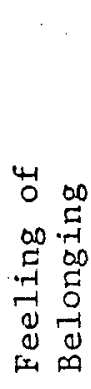 & 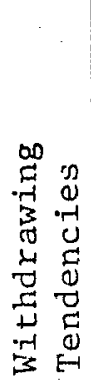 & $\begin{array}{l}E \\
0 \\
\Delta \\
0 \\
0 \\
0 \\
0 \\
\vec{z} \\
0 \\
0 \\
0 \\
0 \\
z\end{array}$ & 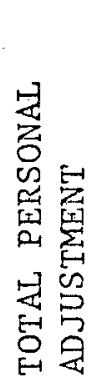 & 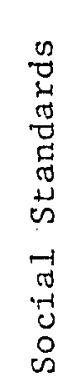 & 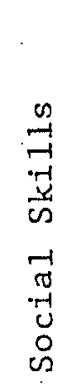 & 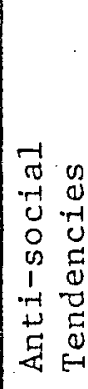 & 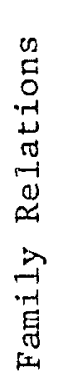 & 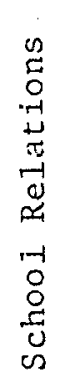 & 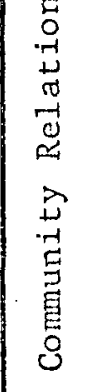 & 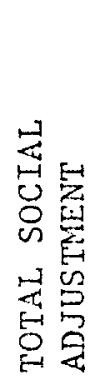 & \\
\hline Reading & .24 & .32 & -.11 & $\begin{array}{r}* \\
.39\end{array}$ & .41 & .22 & $\begin{array}{l}* \% \\
.43\end{array}$ & .18 & .30 & .25 & .11 & .22 & -.03 & .25 & $\begin{array}{r}* \\
.36\end{array}$ \\
\hline Math & .38 & .10 & .06 & .25 & .21 & .12 & .30 & .11 & .13 & .30 & .01 & .12 & -.08 & .15 & .24 \\
\hline Language & .27 & .20 & .08 & .08 & .14 & -.15 & .16 & .03 & .16 & .21 & .07 & .15 & -.01 & .15 & .17 \\
\hline TOTAL BATTERY & .25 & .08 & -.05 & .30 & .29 & .03 & .25 & .14 & .21 & .28 & .02 & .18 & -.08 & .19 & .24 \\
\hline
\end{tabular}

$$
\mathbb{N}=31
$$

$* \mathrm{p}<.05$

$* * \mathrm{P}<.02$ 
TABLE 6

THE CORRELATION BETWEEN SELF-CONCEPT SCORES AND SCHOOL ACHIEVEMENT SCORES LEVEL 6

\begin{tabular}{|c|c|c|c|c|c|c|c|c|c|c|c|c|c|c|c|}
\hline & \multicolumn{7}{|c|}{ PERSONAL ADJUSTMENT } & \multicolumn{7}{|c|}{ SOCTAL ADJUSTMENT } & \multirow[b]{2}{*}{ 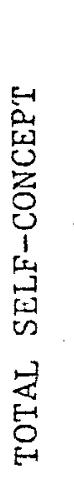 } \\
\hline $\begin{array}{c}\text { SCHOOL } \\
\text { ACHIEVEMENT }\end{array}$ & 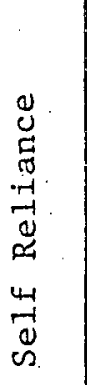 & 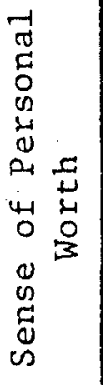 & 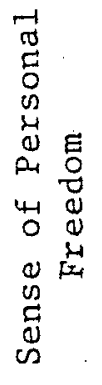 & 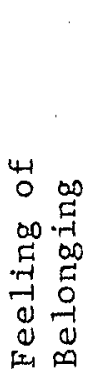 & 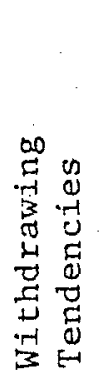 & 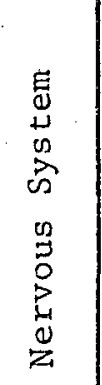 & 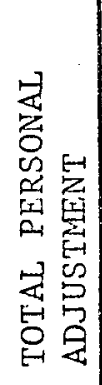 & 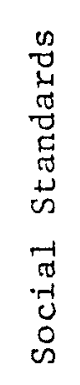 & $\begin{array}{l}n \\
ت \\
= \\
= \\
0 \\
0 \\
-1 \\
\pi \\
-1 \\
0 \\
0 \\
\infty\end{array}$ & 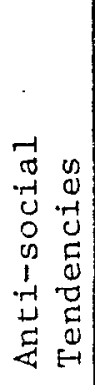 & 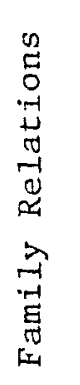 & $\begin{array}{l}0 \\
0 \\
0 \\
-1 \\
-1 \\
0 \\
-1 \\
0 \\
04 \\
-1 \\
0 \\
0 \\
0 \\
0 \\
0 \\
0\end{array}$ & 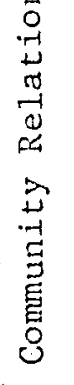 & 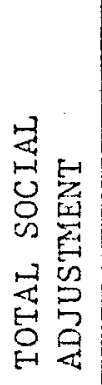 & \\
\hline Reading & .12 & $\begin{array}{r}* \\
.34\end{array}$ & .16 & .30 & -.00 & -.02 & .20 & .18 & .15 & .06 & .10 & .09 & .32 & .23 & .26 \\
\hline Math & .01 & .28 & $\begin{array}{l}.36 \\
* * *\end{array}$ & $\begin{array}{r}.40 \\
* * *\end{array}$ & -.03 & .03 & .25 & $\begin{array}{r}.19 \\
* *\end{array}$ & $\begin{array}{l}.20 \\
* *\end{array}$ & .07 & .23 & .02 & $\begin{array}{l}.30 \\
x * 2 * 5\end{array}$ & $\begin{array}{l}.23 \\
* 2 * x\end{array}$ & $\begin{array}{r}.29 \\
* * x\end{array}$ \\
\hline Language & .01 & .22 & $\begin{array}{r}.50 \\
* *\end{array}$ & $\begin{array}{r}.42 \\
* *\end{array}$ & -.02 & -.04 & .27 & .40 & .42 & .17 & .32 & .20 & $\begin{array}{r}.48 \\
* *\end{array}$ & $\begin{array}{r}.47 \\
x\end{array}$ & .45 \\
\hline TOTAL BATTERY & .01 & .29 & .40 & .42 & -.00 & .01 & .28 & .29 & .28 & .12 & .25 & .11 & .40 & .35 & .38 \\
\hline
\end{tabular}

$N=35$

$* \mathrm{p}<.05$

$* * \mathrm{P}<.02$

$* * * \mathrm{P}<.01$ 
Anarysis of the data for the interactions of level and sex

Lever 4, males: Table 7 indicates that the correlation coefficients for wales in this level ranged from -.25 to .33 . Though most of these correlations were positive, none were statistically significant.

Level 4, females: Table 8 does not indicate a statistically significant correlation between the total self-concept of the females in Level 4 and their total school achievement. However, it does indicate a positive and significant correlation between their total social adjustment and total school achievement $(r=.54, p<.05)$. Further, it indicates a number of positive and statistically significant correlations between reading achievement and withdrawing tendencies, social standards, anti-social tendencies, and community relations.

Level 5, males: Although I'able 9 does not indicate a statisticaliy significant correlation between total self-concept and total school achievement, there were an additional number of fairly high correlations in the data. These correlations, however, were not statistically significant. Level 5, females: For the most part the correlations in Table 10 are not statistically significant. The single significant correlation is found between total personal adjustment and reading achievement $(r=.58, p<.05)$.

Level 6, males: There were no significant correlations between total self-concept and total school achievement in the data in Table 11 , but a significant correlation existed between total social adjustment and language achievement $(\mathrm{r}=.51, \mathrm{p}<.05)$. 
TABLE 7

THE CORRELATION BETWEEN SELF-CONCEPT SCORES AND SCHOOL ACHIEVEMENT SCORES LEVEL 4, MALES

\begin{tabular}{|c|c|c|c|c|c|c|c|c|c|c|c|c|c|c|c|}
\hline \multirow[b]{2}{*}{$\begin{array}{c}\text { SCHOOL } \\
\text { ACHIEVEMENT }\end{array}$} & \multicolumn{7}{|c|}{ PERSONAL ADJUSTMENT } & \multicolumn{7}{|c|}{ SOCIAL ADJUSTMENT } & \\
\hline & 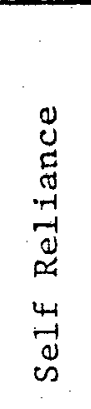 & 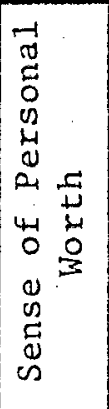 & 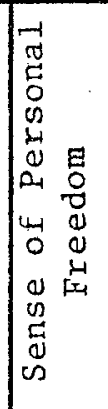 & 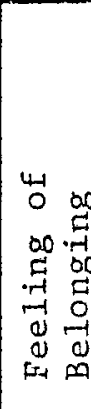 & 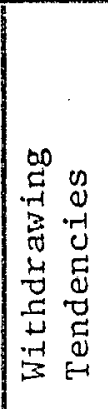 & 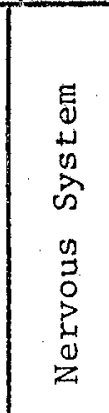 & 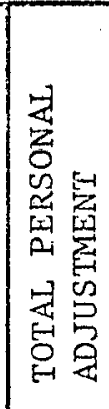 & 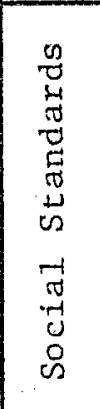 & 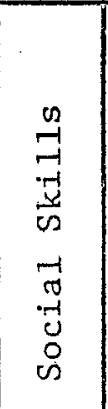 & 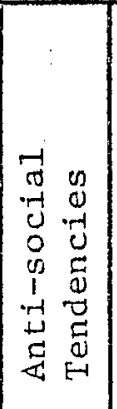 & 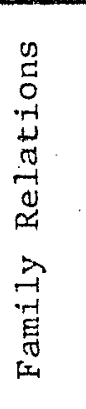 & 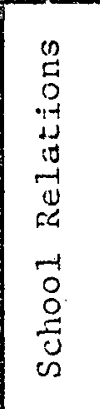 & 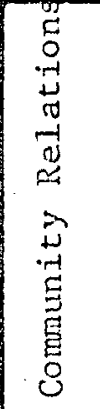 & 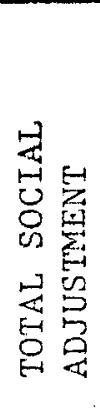 & 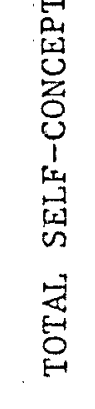 \\
\hline Reading & .22 & -.05 & -.14 & .14 & -.01 & -.24 & -.05 & .18 & -.18 & $-.10 \mid$ & .19 & -.10 & .05 & .04 & -.01 \\
\hline Math & .25 & .22 & .18 & .40 & .12 & -.00 & .25 & .07 & -.15 & $-.05 \mid$ & .18 & .08 & .07 & .02 & .17 \\
\hline Language & .33 & .09 & .09 & .24 & .03 & .08 & .17 & .30 & $-.25 \mid$ & -.01 & .30 & -.02 & -.21 & .02 & .12 \\
\hline TOTAL BATTERY & .24 & .10 & .05 & .25 & .03 & -.07 & .12 & .18 & -.25 & $-.03 \mid$ & .24 & -.02 & -.05 & .01 & .08 \\
\hline
\end{tabular}

$\mathrm{N}=23$ 
TABLE 8

THE CORRELATION BETWEEN SELF-CONCEPT SCORES AND SCHOOL ACHIEVEMENT SCORES LEVEL 4, FEMALES

\begin{tabular}{|c|c|c|c|c|c|c|c|c|c|c|c|c|c|c|c|}
\hline \multirow[b]{2}{*}{$\begin{array}{c}\text { SCHOOL } \\
\text { ACHIEVEMENT }\end{array}$} & \multicolumn{7}{|c|}{ PERSONAL ADJUSTMENT } & \multicolumn{7}{|c|}{ SOCIAL ADJUSTMENT } & \multirow[b]{2}{*}{ 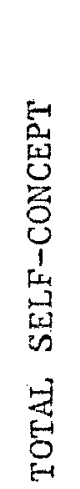 } \\
\hline & 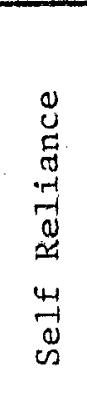 & 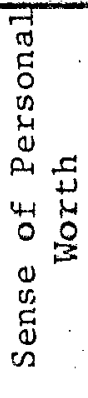 & 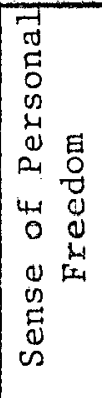 & 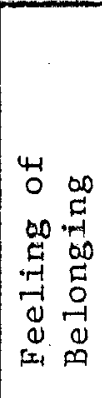 & 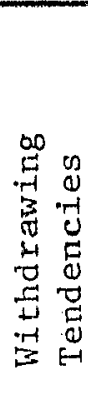 & 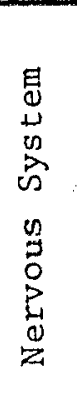 & 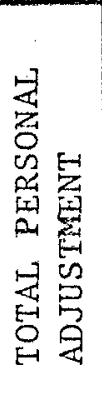 & 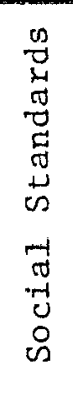 & 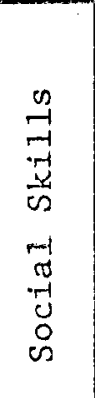 & 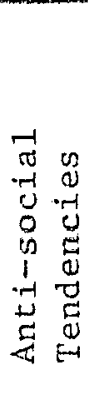 & 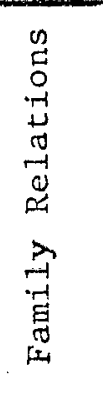 & 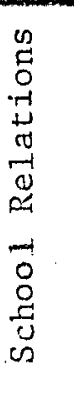 & 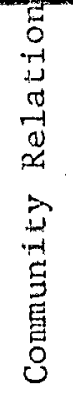 & 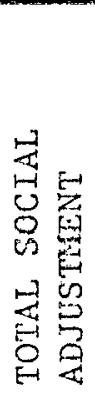 & \\
\hline Reading & .17 & .40 & -.01 & .32 & $\begin{array}{l}x * x \\
.60\end{array}$ & .36 & $\begin{array}{r}* \\
.52\end{array}$ & $\begin{array}{l}\text { *xis } \\
.65\end{array}$ & -.17 & $\begin{array}{l}* \% \\
.57\end{array}$ & .08 & .38 & $\begin{array}{l}* x * x \\
.70\end{array}$ & $\begin{array}{l}\text { *wit } \\
.67\end{array}$ & $\begin{array}{l}* x+\pi \\
.65\end{array}$ \\
\hline Math & -.02 & .17 & -.28 & -.13 & .05 & .36 & .09 & .29 & -.06 & .20 & -.16 & .28 & .49 & .32 & .21 \\
\hline Language & .01 & .28 & .04 & .28 & .33 & .41 & .40 & .03 & -.05 & .35 & .15 & .63 & .11 & .38 & .43 \\
\hline TOTAL BATTERY & .08 & .33 & -.14 & .09 & .37 & .37 & .33 & .60 & -.15 & .47 & -.13 & .29 & .72 & .54 & .47 \\
\hline
\end{tabular}

$\mathrm{N}=16$

$* \mathrm{P}<.05$

$* * \mathrm{P}<.02$

$* * * \mathrm{P}<.01$ 
TABLE 9

THE CORRELATION BETWEEN SELF-CONCEPT SCORES AND SCHOOL ACHIEVEMENT SCORES LEVEL 5, MALES

\begin{tabular}{|c|c|c|c|c|c|c|c|c|c|c|c|c|c|c|c|}
\hline \multirow[b]{2}{*}{$\begin{array}{c}\text { SCHOOL } \\
\text { ACHIEVEMENT }\end{array}$} & \multicolumn{7}{|c|}{ PERSONAL ADJUSTMENT } & \multicolumn{7}{|c|}{ SOCIAL ADJUSTMENT } & \\
\hline & 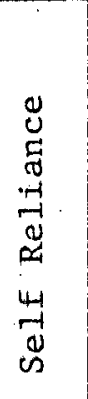 & 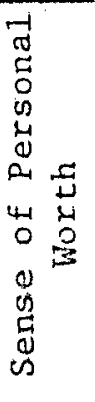 & 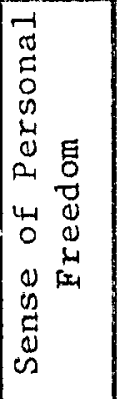 & 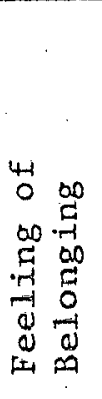 & 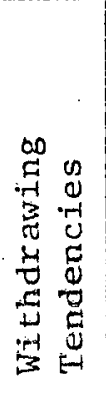 & 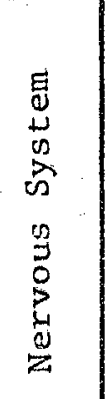 & 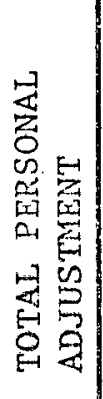 & 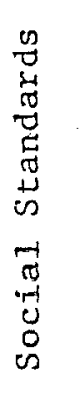 & 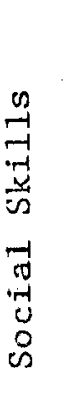 & 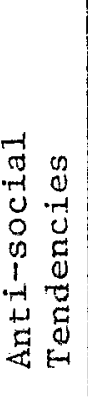 & 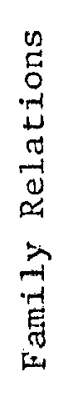 & 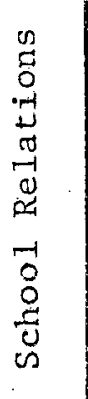 & 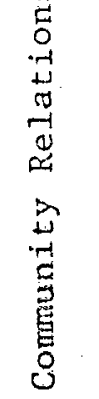 & 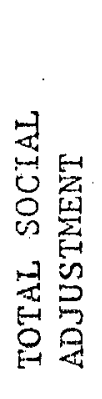 & 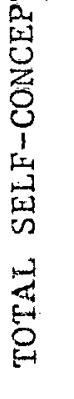 \\
\hline & $\begin{array}{r}.16 \\
*\end{array}$ & .23 & -.32 & .44 & .34 & -.01 & .34 & .31 & .28 & $\begin{array}{r}.33 \\
* *\end{array}$ & .18 & .34 & .02 & .34 & .38 \\
\hline Math & .50 & .25 & -.06 & .26 & .20 & .05 & .46 & .26 & .24 & .58 & .21 & .26 & -.09 & .36 & .44 \\
\hline Language & .34 & .30 & .02 & .16 & .20 & -.28 & .26 & .11 & .16 & .25 & .05 & .35 & -.13 & .20 & .25 \\
\hline TOTAL BATTERY & .36 & .27 & -.09 & .33 & .30 & -.11 & .40 & .26 & .24 & .44 & .16 & .36 & -.06 & .34 & .41 \\
\hline
\end{tabular}

$$
\mathrm{N}=16
$$

$$
\begin{aligned}
& * \mathrm{P}<.15 \\
& * * \mathrm{P}<.02
\end{aligned}
$$


TABLE 10

THE CORRELATION BETWEEN SELF-CONCEPT SCORES AND SCHOOL ACHIEVERTNT SCORES LEVEL 5, FEMALES

\begin{tabular}{|c|c|c|c|c|c|c|c|c|c|c|c|c|c|c|c|}
\hline & \multicolumn{7}{|c|}{ PERSONAI ADJUSTMENT } & \multicolumn{7}{|c|}{ SOCIAL ADJUSTMENT } & \multirow[b]{2}{*}{ 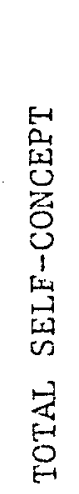 } \\
\hline $\begin{array}{c}\text { SCHOOL } \\
\text { ACHIEVEMENT }\end{array}$ & 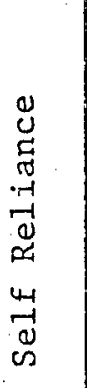 & 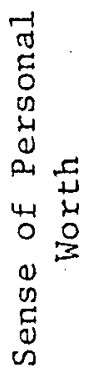 & 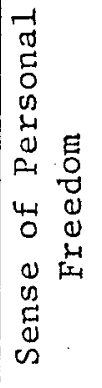 & 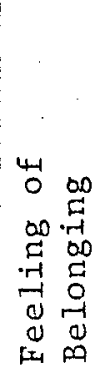 & 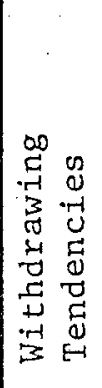 & 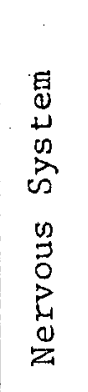 & 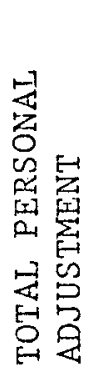 & 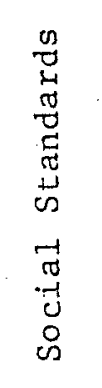 & 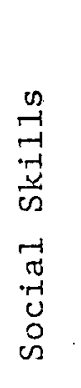 & 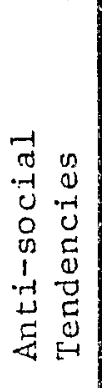 & 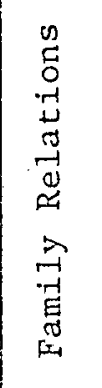 & $\begin{array}{l}0 \\
0 \\
0 \\
-1 \\
D \\
0 \\
0 \\
0 \\
\sim \\
-1 \\
0 \\
0 \\
0 \\
0 \\
0 \\
0\end{array}$ & 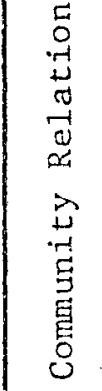 & 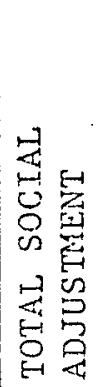 & \\
\hline Reading & .34 & .47 & .10 & .42 & .49 & .41 & .58 & .01 & .32 & .19 & .06 & .10 & -.07 & .17 & .41 \\
\hline Math & .22 & .04 & .27 & .32 & .24 & .18 & .30 & -.08 & .04 & .02 & -.18 & -.02 & 5.06 & -.06 & .13 \\
\hline Language & .21 & .06 & .08 & -.07 & .07 & -.03 & .07 & -.09 & .18 & .16 & .05 & -.14 & -.08 & .04 & .06 \\
\hline TOTAL BATTERY & .26 & .18 & .13 & .24 & .27 & .24 & .33 & -.05 & .14 & .11 & -.06 & -.04 & -.09 & .02 & .19 \\
\hline
\end{tabular}

$\mathrm{N}=15$

$* \mathrm{P}<.05$ 
TABLE 11

THE CORRELATION BETWEEN SELF-CONCEPT SCORES AND SCHOOL ACHIEVEMENT SCORES LEVEL 6, MALES

\begin{tabular}{|c|c|c|c|c|c|c|c|c|c|c|c|c|c|c|c|}
\hline \multirow[b]{2}{*}{$\begin{array}{c}\text { SCHOOL } \\
\text { ACHIEVEMENT }\end{array}$} & \multicolumn{7}{|c|}{ PERSONAL ADJUSTMENT } & \multicolumn{7}{|c|}{ SOCIAL ADJUSTMENT } & \\
\hline & 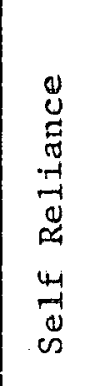 & 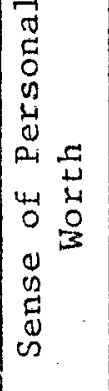 & 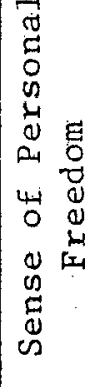 & 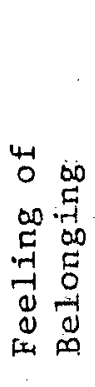 & 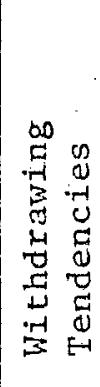 & 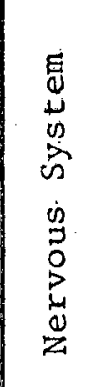 & 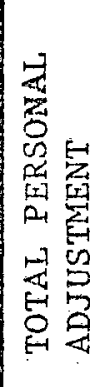 & 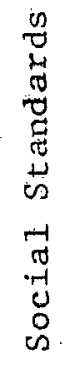 & 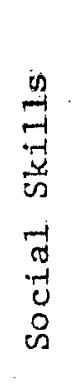 & 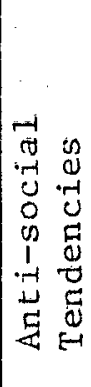 & 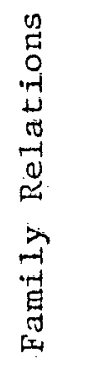 & 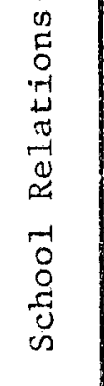 & 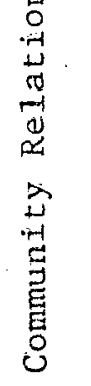 & 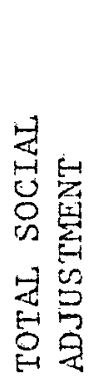 & 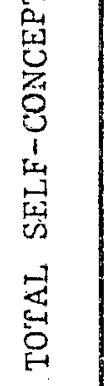 \\
\hline Reading & .26 & .17 & .04 & .22 & -.05 & .13 & -.03 & .16 & .16 & -.03 & .07 & -.29 & .28 & .06 & .14 \\
\hline Math & -.04 & .25 & .19 & .36 & -.22 & -.09 & .11 & $\begin{array}{l}.52 \\
* * * *\end{array}$ & .15 & .12 & :16 & -.02 & $\begin{array}{r}.32 \\
*\end{array}$ & $\begin{array}{r}.28 \\
*\end{array}$ & \\
\hline Language & .11 & .02 & .31 & .32 & -.17 & .03 & .14 & .69 & .35 & .15 & .35 & .15 & .49 & .51 & \\
\hline TOTAL BATTERY & .11 & .14 & .25 & .34 & -.18 & .06 & .17 & .59 & .17 & .11 & .24 & -.01 & .44 & .36 & .33 \\
\hline
\end{tabular}

$$
\mathrm{N}=18
$$

$* P<.05$

$* * \mathrm{P}<.02$

is* $\mathrm{P}<.01$ 
Level 6, females: Like rables 10 and 11 , Table 12 does not indicate statistical significance between the composite score of self-concept and the composite score of school achievement. A significant correlation, however, existed between sense of personal freedom and language achievement $(\mathrm{r}=.61, \mathrm{p}<.01)$.

\section{Summary of the Findings}

The hypotheses of the study were as follows:

1. A positive correlation exists between the self-concept of the disadvantaged child and his school achievement.

2. There are no differences between males and females in the correlation between the self-concept and school achievement.

3. There are no differences between school placement levels in the correlation between the self-concept and school achievement.

General: When sex and school placement level were factored out, the data indicated a positive and statistically significant correlation between the composite self-concept scores and the composite academic achievement scores. Hypothesis No. 1 was thus supported to this extent.

Sex Differences: The analysis of the data also indicated that when placement level was held constant and sex was allowed to vary the males had a significant correlation between their composite self-concept scores and their composite academic achievement scores. These correlations, however, were not significant for the females. Hypothesis No. 2 was both supported and rejected to this extent. 
TABLE 12

THE CORRELATION BETWEEN SELF CONCEPT SCORES AND SCHOOL ACHIEVEMENT SCORES LEVEL 6, FEMALES

\begin{tabular}{|c|c|c|c|c|c|c|c|c|c|c|c|c|c|c|c|}
\hline & \multicolumn{7}{|c|}{ PERSONAL ADJUSTMENT } & \multicolumn{7}{|c|}{ SOCIAL ADJUSTMENT } & \multirow[b]{2}{*}{ 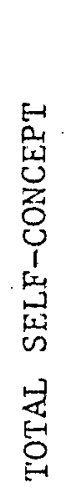 } \\
\hline $\begin{array}{c}\text { SCHOOL } \\
\vdots \\
\text { ACHIEVEMENT }\end{array}$ & 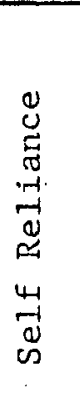 & $\begin{array}{l}\overrightarrow{7} \\
\pi \\
0 \\
0 \\
0 \\
0 \\
0 \\
01 \\
4 \\
4 \\
0 \\
0 \\
0 \\
0 \\
0 \\
0 \\
0 \\
0 \\
0\end{array}$ & 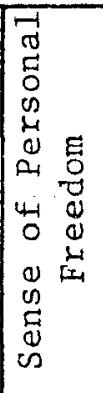 & 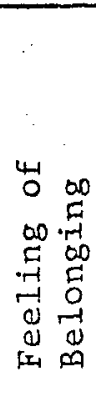 & 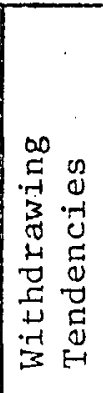 & $\begin{array}{l}\tilde{E} \\
0 \\
\Delta \\
0 \\
D \\
0 \\
0 \\
0 \\
0 \\
0 \\
0 \\
0 \\
z\end{array}$ & 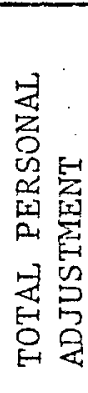 & 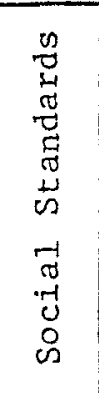 & 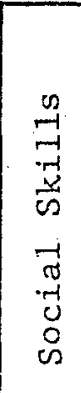 & 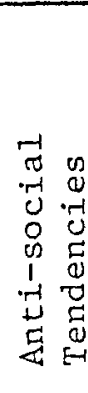 & 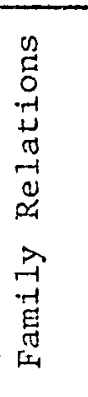 & 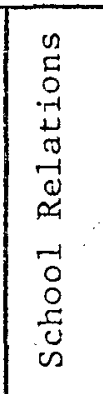 & 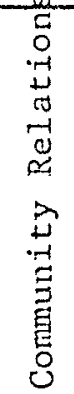 & 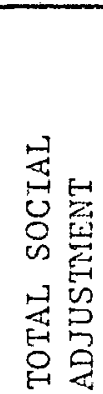 & \\
\hline Reading & .11 & .34 & .14 & .32 & .17 & .21 & .30 & -.10 & .00 & .16 & .09 & .22 & .24 & .15 & .26 \\
\hline Math & .06 & .24 & & .43 & .15 & .30 & .39 & -.23 & .14 & .05 & .28 & -.06 & .24 & .10 & .30 \\
\hline Language & .14 & .26 & .61 & .48 & .20 & .19 & .45 & -.06 & .33 & .22 & .28 & .08 & .39 & .29 & .43 \\
\hline TOTAL BATTERY & .14 & .30 & .45 & .47 & .21 & .26 & .44 & .15 & .18 & .17 & .26 & .06 & .30 & .20 & .37 \\
\hline
\end{tabular}

$\mathrm{N}=17$

$* \mathrm{P}<.05$

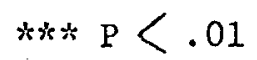


Leve1 differences: When the sex of the subjects was held constant while allowing placement level to vary, a positive and significant correlation between the composite self-concept scores and the composite school achievement scores was indicated for the Level 6 , but not for the Leve1 4 and 5 subjects. Thus Hypothesis No. 3 was both supported and rejected to this extent.

Interaction of sex and placement level: Further, the data indicated that when both sex and placement level were varied no significant correlations were indicated between the total self-concept and total academic achievement. However, this does not mean that there was no correlation whatsoever, since in Tables 6 to 12 a number of specific evidences can be seen which are suggestive of associations between self-concept and school achievement. Although the correlation coefficients were frequently high when sex and placement levels were varied together, the number of subjects involved in the specific sex placement groups was low. The N's in these cases ranged from 13 to 21 . This made statistically significant coefficients much more difficult to obtain due to the correction factors inherent in the correlational procedures.

The analysis also indicated that certain areas of the self-concept produced a large number of significant correlations with specific areas of school achievement. For example, "feeling of belonging" and "social standards" were self-concept factors which were frequently significantly correlated with academic achievement.. On the other hand, "family relations". and "school relations" showed no statistical significance for any of the correlations. Other findings were that reading achievement was 
frequently significantly correlated with personal adjustment and that language achievenent was frequently significantly correlated with social adjus tment.

It is important to note that all the statistically significant correlations in the study were positive, demonstrating that achievement was "satisfactory" when the self-concept was "satisfactory," and that achievement was "unsatisfactory" when the self-concept was "unsatisfactory". 
CHAPTER V

CONCLUSIONS AND IMPLICATIONS

\section{Conclusions}

The data analyzed in chapter IV indicates that a relationship exists between the self-concept and the school achievement of the disadvantaged child. The fact that this relationship was always positive whenever it was statistically significant is of importance. It indicates that achievement was "satisfactory" when the self-concept was "satisfactory", and that achievement was "unsatisfactory" when the self-concept was "unsatisfactory". When a child had a poor self-concept his school achievement was low, and when he had a good self-concept his school achievement was high.

Further, the results indicate that the relationship between the self-concept and school achievement is positive and significant in males, but that the relationship is not significant in females. This finding is in general agreement with the findings of Bledsoe (1967): "Correlations of achievement and SC (self-concept) for boys were significant and positive; for girls they were non-significant." Bledsoe suggested that such a difference "may be a function of maturation, since girls of ages nine to eleven are on the average more developed than boys. Another reason may be that the more frequent contacts with women teachers and with mothers (compared with fathers) enables girls to develop a more satisfying self-image at these developmental stages." 
It may also be that boys have a different attitude in the test situation. They may be more ready and willing to admit their faults than girls.

The results of the analysis also indicate a significant relationship between the self-concept and school achievement for subjects in school placement Level $\epsilon$, but that this relationship is not significant for the other two levels. This is in general agreement with the findings of Piers and Harris (1964) who found that the relationship between selfconcept scores and acadenic achievement scores was greater at the sixth grade than at the third grade level. This may indicate that the selfconcept is constantly changing and developing side by side with academic achievement, and that whichever direction one takes the other one follows. Another result of the analysis of data was that reading achievement was more correlated with aspects of the personal adjustment, whereas language achievement was more correlated with aspects of social adjustment. This is not surprising, especially when we find that the major function of language is to provide the individual with the means for the establishment of interpersonal relations. Gordon (1969) also sees the relation of social adjustment with language achievement, for she suggests that a deprived environment retards language development. Reading is more personal, and here Gordon suggests that what children think of themselves affects their reading achievement.

Two factors of the self-concept test stood out as having a greater number of significant relationships with achievement than the other 10 factors had. These two factors were "feeling of belonging" and "social standards." Both of these self-concept factors are found to constitute 
the self-concept according to Woolner (1971): "It (self-concept) consists of the traits, attributes, and limitations the individual assigns to himself (his self-concept); the individual's evaluation of himself (his ideal self-concept); and how the individual perceives what others think of him (his other self-concept)." Thus the general finding of the present study is in agreement with Woolner's definition of the self-concept.

\section{Implications}

Basic to the acquisition of adequate and accurate self-concepts is the teacher, the adult, or the significant other who accepts each child as a unique person who is worthy of one's concern no matter how much or how little he can give.

Also basic to the acquisition of adequate self-concepts are educators, who by example help their pupils see in them individuals who are interested and sincere. Individuals who are honest, trustworthy and who themselves possess positive self-concepts.

The findings of the present study are very important because they are predictive. They predict that a child with a poor self-concept will perform poorly in reading, mathematics, and language. They also predict that a child with a good self-concept will perform at a higher level in reading, mathematics, and language.

The implication of this study is that educators must:

1. Provide opportunities for children in experiences that will enhance their self-acceptance, their acceptance of others, and their seif-understanding.

2. Help students to attain realistic goals and meet conflict situations. 
3. Develop the curriculum around meeting the psychological needs as well as the academic needs of children.

4. Make provision for the services of an educational psychologist in the school system, especially where there are schools for the disadvantaged.

5. Train teachers in methods that will help them in meeting the psychological as well as the academic needs of their pupils.

\section{Turn their attention to the development of healthy self-} concepts, in addition to the development of attitudes, values, and cultures germane to the children.

7. Conduct similar research on self-concept and school achievement in students from non-disadvantaged areas to see if the relationship between them generalizes across all socio-economic levels.

The data of this study indicate that: (1) there is a positive and statistically significant correlation between the self-concept and academic achievement, (2) self-concept scores are predictive and show that the lower the self-concept in disadvantaged children the lower will be their school achievement, and that the higher the self-concept the higher will be their school achievement. This means that when educators attempt to meet the self-concept needs as well as the academic needs of their pupils, success will be much more likely in our schools. 
APPENDIX A

CALIFORNIA ACHIEVEMENT TEST 


\section{Complete Battery}

READING • MATHEMATICS - LANGUAGE

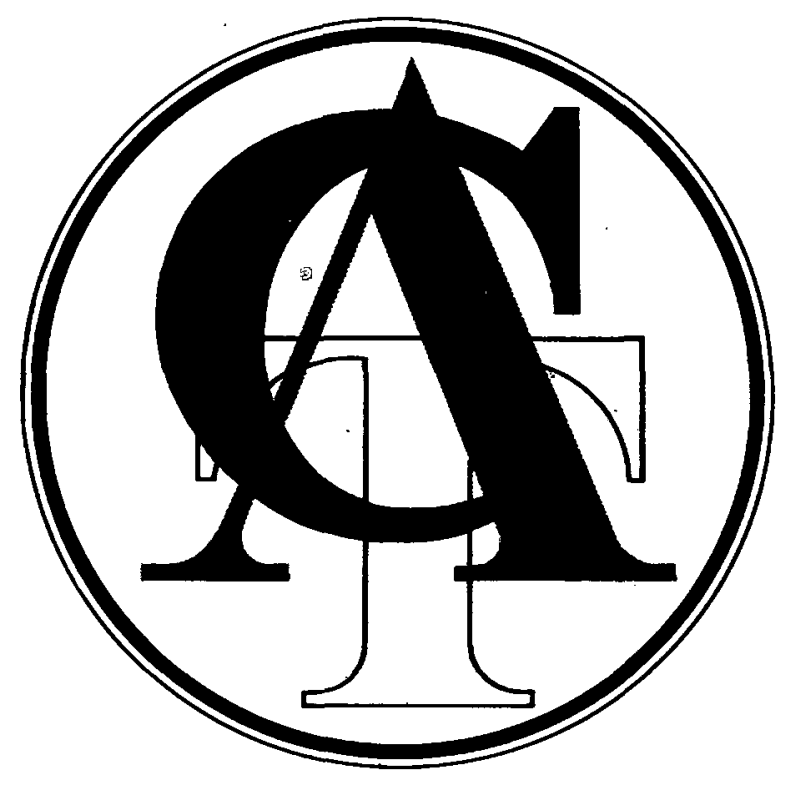

1970 EDITION
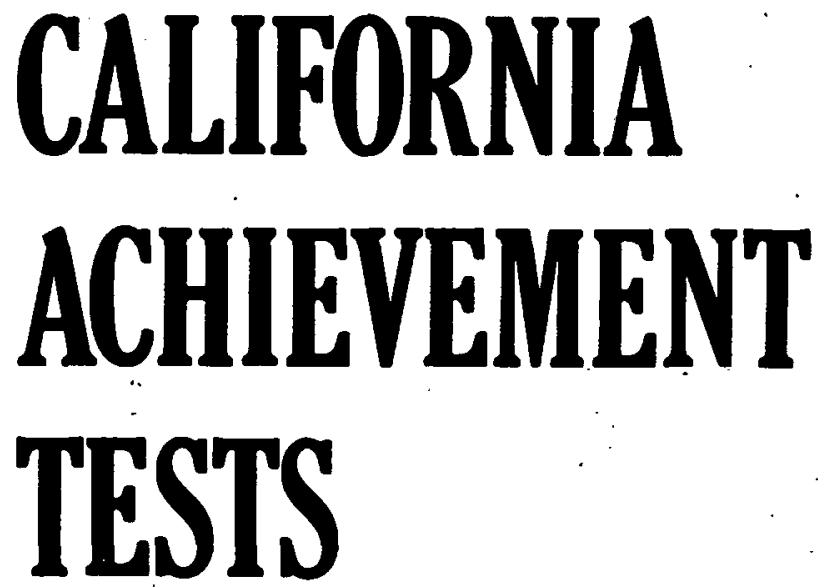

Devised by ERNEST W. TIEGS AND WILLIS W..CLARK Published by CTB/McGraw-Hill, Del Monte Research Park, Monterey, California 93940. Copyright $\bigodot_{1957,1963,1971 \text { by McGraw-Hill, Inc. }}$ All Rights Reserved. Printed in the U.S.A. No part of this publication may be reproduced, stored in a retrieval system, or transmitted, in any
All form or by any means, electronic, mechanical, photocopying, recording, or otherwise, without the prior written permission of the publisher. 
This booklet contains tests that measure your ability to use basic skills that are important in many things you do every day. The paragraphs below give you some points to remember while you take these tests.

Make sure you understand all the directions before you begin each section. You may ask questions about any directions you do not understand. Do not begin any section until you are told to do so.

0 Work as fast as you can. There may be items you cannot do because they test things you have not yet been taught. If an item is too difficult, do not spend too much time on it. Make the most careful choice you can and go on to the next item.

$O$ If you come to the word STOP at the bottom of a page before time is called, do not turn the page. Go back over your work in the section you have just completed.

Mark all your answers on the answer sheet. Answer marks should be neat and clear. Be sure you mark only one answer for each item, unless you are specifically told otherwise: If you make a mistake or want to change an answer, erase your first answer completely.

CTB/McGraw-Hill is indebted to the following for permission to use selections which appear in this test booklet.

Page 10, text material adapted from "Wheat's Origin is Unknown, But It's Grown Everywhere," by the U.S. Department of Agriculture, with permission of The Farm Index, December 1968, reprinted in the Journal of Geography, May 1969, Vol. LXVIII, No. 5.

Page 12, text material adapted by permission of Webster Division, McGraw-Hill Book Company, "Life Histories of Other Insects," Teacher's Manual for Life Histories, by Harold E. Tannenbaum, Beulah Tannenbaum, Nathan Stillman, and Myra Stillman, 1967.

Major credit for the development of the 1970 edition of this test booklet belongs to R. Alexander Long, Ph.D., . Senior Project Director; Doris Penberthy, M.S., Associate Editor; Sherry Dixon, M.A., Editing Supervisor; Susan Jones, M.A., Consultant; Barbara Bullock, M.A., Consultant; C. Craig Bowen, Designer; and Ralph Brink, Director of Manufacturing. 


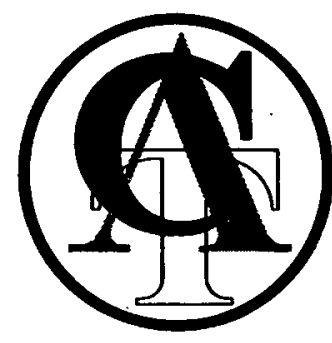

SECTION 1

READING Vocabulary

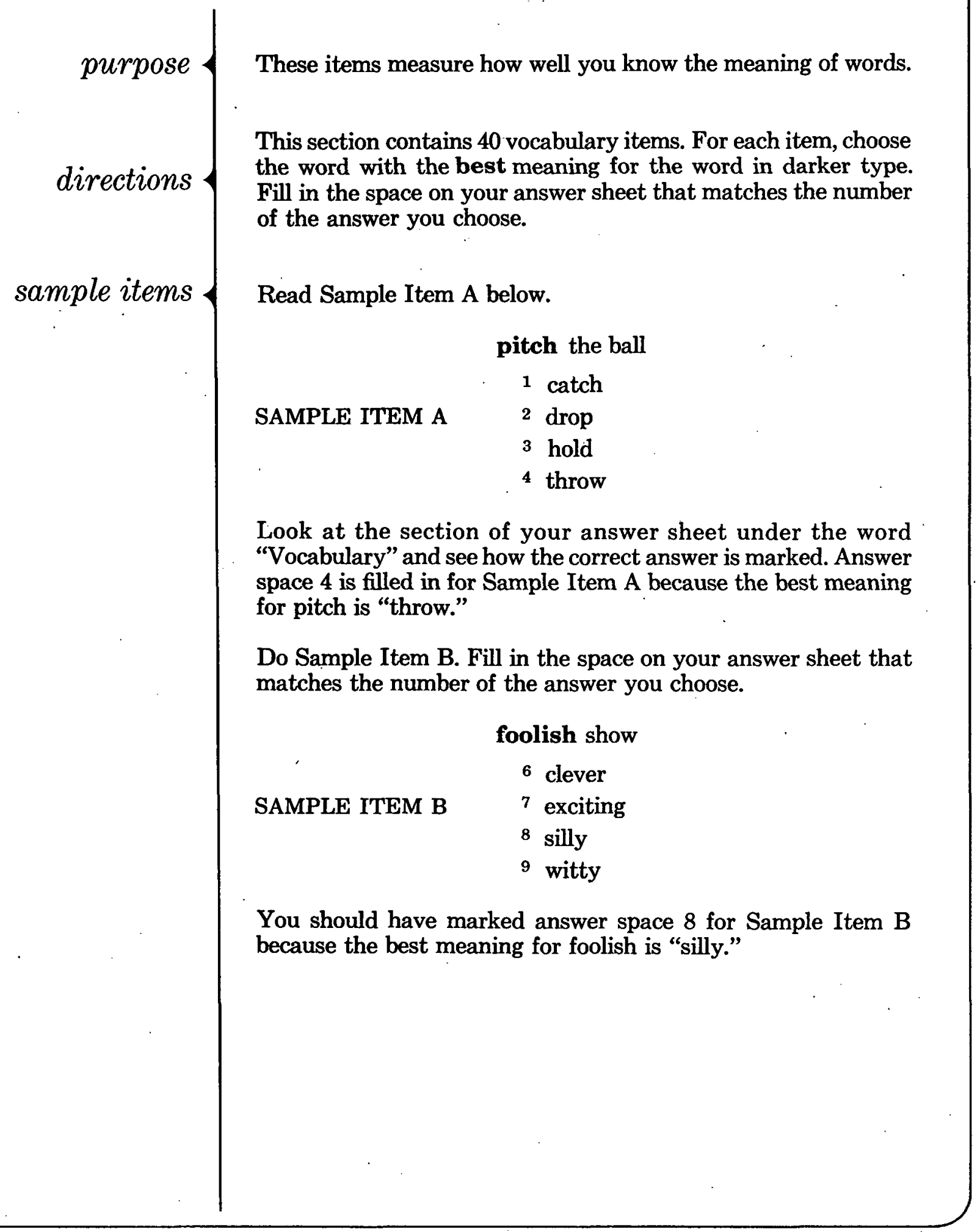


Do Items 1-40 and mark your answers on the answer sheet. Be careful to keep your place on the answer sheet.

1 select a book

$$
\begin{aligned}
& 1 \text { choose } \\
& 2 \text { consider } \\
& 3 \text { skim } \\
& { }^{4} \text { study }
\end{aligned}
$$

\section{2 entire class}

$$
\begin{array}{ll}
6 & \text { enclosed } \\
7 & \text { evening } \\
8 & \text { huge } \\
9 & \text { whole }
\end{array}
$$

3 alert dog

$$
\begin{array}{ll}
1 & \text { amusing } \\
2 & \text { cute } \\
3 & \text { healthy } \\
4 & \text { watchful }
\end{array}
$$

4 search for gold

$$
\begin{array}{ll}
6 & \text { dig } \\
7 & \text { look } \\
8 & \text { send } \\
9 & \text { wait }
\end{array}
$$

5 peaceful day

1 busy

2. calm

3 successful

4 tiresome

6 combine seeds

6 change

7 compare

8 mix

9 taste
7 three paces

$$
\begin{array}{ll}
1 & \text { packages } \\
2 & \text { pads } \\
3 & \text { races } \\
4 & \text { steps }
\end{array}
$$

8 courteous manner

$$
\begin{array}{ll}
6 & \text { brave } \\
7 & \text { cruel } \\
8 & \text { polite } \\
9 & \text { shy }
\end{array}
$$

9 thorough inspection

$$
\begin{array}{ll}
1 \text { discussion } \\
2 \text { examination } \\
3 \text { initiation } \\
4 \text { instruction }
\end{array}
$$

10 valuable jewels

6 beautiful

7 genuine

8 old

9 precious

11 clumsy movement
1 anxious
2 awkward
3 backward
4 graceful

12 dry region
6 area
7 asphalt
8 refuge
9 weather

13 item of value

$$
\begin{array}{ll}
1 & \text { article } \\
2 & \text { furniture } \\
3 & \text { invitation } \\
4 & \text { position }
\end{array}
$$

14 large cushion

$$
\begin{array}{ll}
6 & \text { carriage } \\
7 & \text { drapes } \\
8 & \text { pillow } \\
& \text { ruffle }
\end{array}
$$

15 postpone action

$$
\begin{array}{ll}
1 & \text { delay } \\
2 & \text { demand } \\
3 & \text { forbid } \\
& \\
4 & \text { prevent }
\end{array}
$$

16 gray substance

6 carpeting

7 mansion

8 material

9 surface

17 notify the group

1 confuse

2. inform

3 neglect

4 observe

18 aided a family

6 adopted

7 entertained

${ }^{8}$ helped

9 raised

19 display the pictures

1 discard

2 exchange

3 exhibit

4 paint 
20 direct the company

6 defend

7 describe

8 leave

9 manage

21 gorgeous view

1 costly

2 gloomy

3 magnificent

4 numerous

22 expressed his opinion
6 excused
7 praised
8 reversed
9 stated

23 flee the storm
1 cause
2 escape
3 ignore
4 watch

24 furnish clothing

$$
\begin{array}{ll}
6 & \text { buy } \\
7 & \text { manufacture } \\
8 & \text { provide } \\
{ }^{9} & \text { sell }
\end{array}
$$

25 transparent glass

$$
\begin{array}{ll}
1 & \text { clear } \\
2 & \text { dark } \\
3 & \text { regular } \\
& \text { rough }
\end{array}
$$

26 probable effect

$$
\begin{array}{l|l}
6 & \text { abrupt } \\
7 & \text { likely } \\
8 & \text { particular } \\
9 & \text { previous }
\end{array}
$$

27 accomplish his aim

$$
\begin{array}{ll}
1 & \text { alter } \\
2 & \text { forget } \\
3 & \text { fulfill } \\
4 & \text { plan }
\end{array}
$$

28 dense forest

$$
\begin{array}{ll}
6 & \text { burnt } \\
7 & \text { damp } \\
8 & \text { green } \\
9 & \text { thick }
\end{array}
$$

29 conclusion of a concert

1 end

2 introduction

3 performance

4 rehearsal

30 elevate the platform

$$
\begin{aligned}
& 6 \text { eliminate } \\
& 7 \text { expand } \\
& 8 \text { mount } \\
& 9 \text { raise }
\end{aligned}
$$

31 interpret the message

$$
\begin{aligned}
& 1 \text { explain } \\
& 2 \text { send } \\
& 3 \text { sign } \\
& 4 \text { write }
\end{aligned}
$$

32 kind deed
6 act
7 donor
8 note
9 remark

33 unusual circumstances

1 ceremonies

2 communications

3 conditions

4 consequences
34 grant approval
6 await
7 deny
8 gain
9 give

35 advantageous location

1 additional

2 alternate

3 customary

4 favorable

36 regulate the temperature
6 adjust
$?$ check
8 increase
9 lower

37 important goal
1 date
2 example
3 purpose
4 suggestion

38 wide obstacle
6 barrier
7 boulevard
8 channel
9 landscape

39 great burden
1 benefit
2 force
3 load
4 loss

40 apply a theory
6 approve
7 propose
8 replace
9 use 


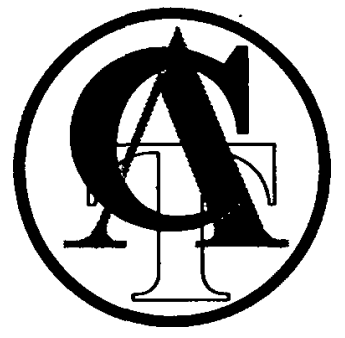

SECTION 2

READING Comprehension

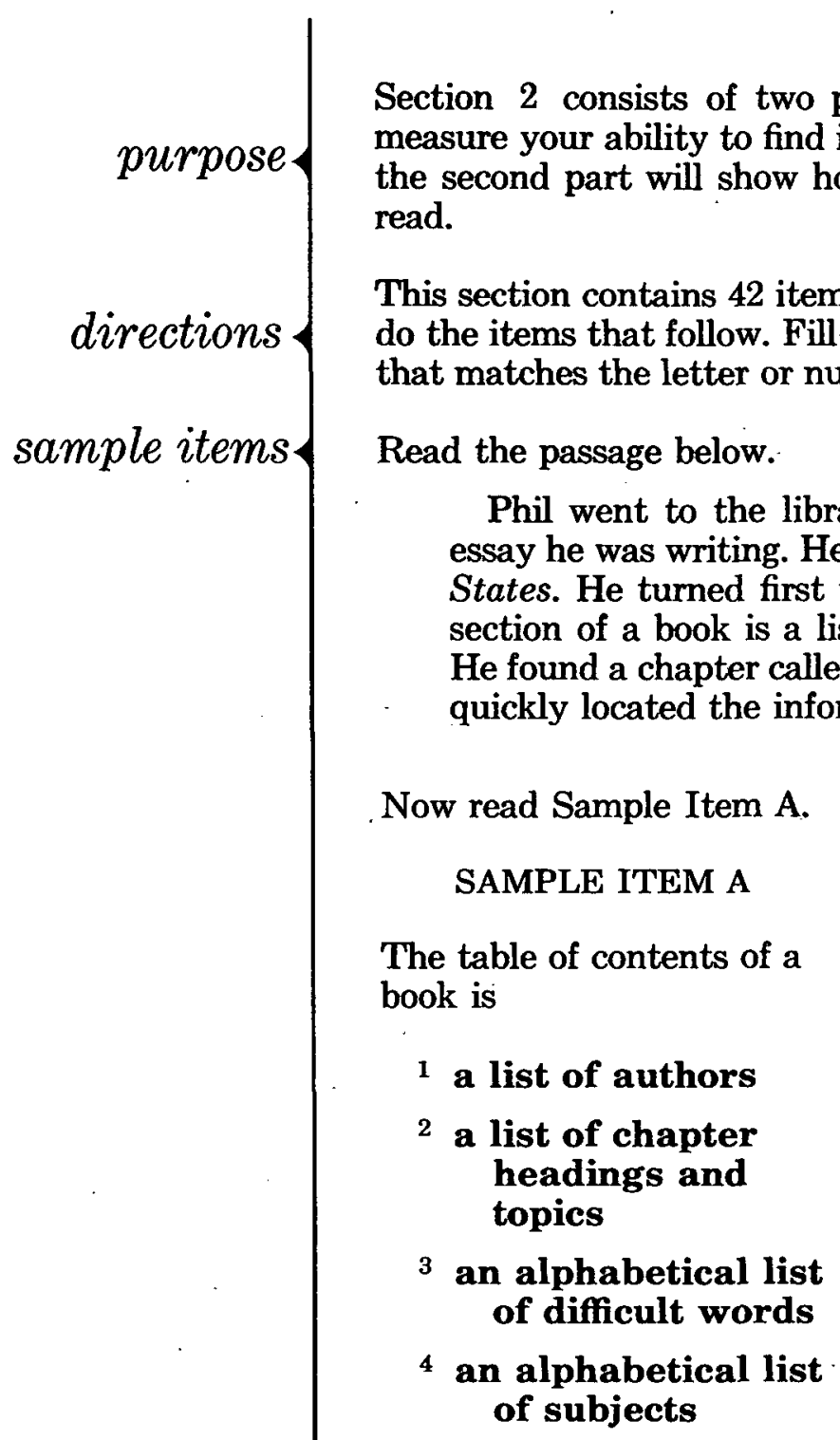

Look at the section of your answer sheet under the word "Comprehension" and see how the correct answer is marked. Answer space 2 is filled in for Sample Item A because the table of contents of a book is "a list of chapter headings and topics."
Do Sample Item B. Fill in the space on your answer sheet that matches the number of the answer you choose.

\section{SAMPLE ITEM B}

Hawaii is the name of a

$$
\begin{array}{ll}
6 & \text { city } \\
7 & \text { country } \\
8 & \text { state } \\
9 & \text { village }
\end{array}
$$

You should have marked answer space 8 for Sample Item $B$ because Hawaii is the name of a "state." 
Read the table of contents. Do Items 1-3 and mark your answers on the answer sheet. Be careful to keep your place on the answer sheet.
Read the index. Then do Items 4-6 and mark your answers on the answer sheet.

\section{TABLE OF CONTENTS}

\section{CHAPTER}

PAGE

1. Climate of United States . . . . . . . . 1

2. Fisheries .................. 35

3. Mineral Industries . . . . . . . . . 47

4. Rock and Soil Products . . . . . . . . . 62

5. Great Cities $\ldots \ldots \ldots \ldots \ldots \ldots \ldots$

6. Commercial Growth . . . . . . . . . 96

1 “Mineral Industries" begins on page
A 35
B 47
C 62
D 83

2 The chapter that begins on page 62 is
F "Commercial Growth"
G "Fisheries"
H "Great Cities"
J "Rock and Soil Products"

3 Page 43 belongs in Chapter
A 1.
B 2.
C 3 .
D 4.

\section{INDEX}

Aireraft: air flow, 123 ; engines, 150; heavier-than-air, 160; jet, 172; manufacture of, 191; patents, 132; pilots, 178 ; structures, 155; ventilation, 112.

Animal Industry: beef cattle, 231 ; cows, 210 ; horses, 228 ; mules, 218.

Apples, 251, 266, 268.

Arizona: agriculture, 313;

fishing, 322; government, 340 ; irrigation, 318; manufacturing, 328.

Arrows: arrowheads, 391; arrow poisoning, 385.

Art: ancient, 395; modern, 401.

4 Information about ancient art is on page
F 324
G 385
H 395
J 401

5 Information about aircraft pilots is on page
A 112
B 123
C 132
D 178

6 Information about irrigation in Arizona is on page
F 313
G 318
H 328
J 340 
Read the following stories. After each story choose the best answer for each item and mark your answer sheet.

Mexico, the third largest country in North America, is a land of geographical differences.

Of all her surface features, mountains are the most common. Three mountain ranges, all part of the Sierra Madre, enclose a central plateau. The southern area of this plateau has served as a center of life even before the development of the Mexican state. Located here today is Mexico City, the nation's capital.

Mexico has lengthy coastlines and waters filled with fish. Even so, the mountain ranges and the inland customs have prevented her from developing many ports or maritime industries.

In the north, desert conditions exist. These dry lands support only dull, colorless plánts. No farming is possible without irrigation.

A volcanic belt runs in an east-west direction close to Mexico City. Since volcanic soil is extremely fertile, major agricultural regions have formed near this belt. Another farming region has developed along the Gulf of Mexico where a small band of good soil supports the growth of tropical fruits.

Farther south toward the equator, Mexico turns into a tropical jungle. This dense vegetation adds yet another part to the geographical mosaic that is Mexico.

This story is mainly about

1 Mexico's geography

2 mountain ranges

3 North America

4 The Mexican coastline

8 Without irrigation, no farming would be possible in Mexico's

${ }^{6}$ mountain plateau

7 northern desert

8 tropical jungle

${ }^{9}$ volcanic region
9 Mexico's coastline is

1 heavily populated

2 industrially developed

${ }^{3}$ long

4 straight

10 How many countries in North America are larger than Mexico?
6 zero
7 one
8 two
9 three

11 The part of Mexico with the highest agricultural-production is the

1 coastal belt

2 desert area

3 tropical jungle

4 volcanic region

The central plateau's western boundary is

6 a mountain range

7 a tropical jungle

8 desert land

9 the volcanic belt

13 Mexico's population centers in the

1 northern desert

2 south-central region

${ }^{3}$ southern jungle

${ }^{4}$ western coastal area 
Wheat covers more than 20 per cent of the world's cropland and is grown in nearly every country. However, the origin of this grain still remains a mystery.

The common ancestor of all wheats is believed to be wild einkorn, still found growing in the Balkan States of eastern Europe. Traces of wheat have also been uncovered through the study of the remains of many early civilizations. It was grown, for example, in southeastern Asia, supposedly man's first home. Even with these findings, neither the source of this grain nor how it arrived in the New World has been determined.

Wheat may have reached America with the early Spanish conquerors. Later it became an important crop in the early Virginia colonies along the Atlantic coast. Now the United States produces more types of wheat than any other nation in the world.

Since its beginnings, major improvements in wheat have been accomplished. At first, progress was slow. The best grain from one year's harvest was selected as the next year's seed. In the twentieth century, however, plant scientists have learned to improve wheat by repeated cross-breeding of one kind of wheat with other kinds. This method is called hybridization.

Only three species of wheat are now important commercially: common, club, and durum. These varieties account for 90 per cent of all wheat grown.

14 About how much of the world's cropland does wheat cover?

$$
\begin{array}{rr}
6 & 3 \% \\
7 & 20 \% \\
8 & 40 \% \\
9 & 90 \%
\end{array}
$$

15 Which of the following varieties of wheat is not important in today's market?
1 club
${ }^{2}$ common
3 durum
4 wild einkorn

16 Recent improvements in wheat have resulted mainly from

\footnotetext{
${ }^{6}$ durum wheat seeds

${ }^{7}$ early civilizations

${ }^{8}$ hybridization methods

${ }^{9}$ mysterious origins
} 
17 The common beginning of all wheats is thought to be
1 club
${ }^{2}$ durum
3 wild einkorn
4 wild rye

18 Hybridization is better than planting the best seed from each year's harvest because

6 it doesn't waste seeds

7 it produces more rapid improvement

${ }^{8}$ plant scientists developed it

9 the United States used it first

19 The article states that wild einkorn still grows in

1 Spain

2 the Balkan States

3 the remains of early civilizations

4 Virginia

20 The first and second paragraphs are mainly about

${ }^{6}$ ancient civilizations

7 areas of wheat production

8 the New World

9 the origin of wheat

21 Hybrid wheat was produced by
1 plant scientists
2 Professor Einkorn
3 the Spanish conquerors
4 the Virginia colonists

22 The third paragraph is mainly about the

6 early Virginia colonies

7 history of wheat in the United States

${ }^{8}$ Spanish conquerors

9 varieties of wheat grown in America

23 The article suggests that wheat has a long history since

1 it covers so much of the world's cropland

2 it is grown in the Balkan States

${ }^{3}$ the American colonies raised it

${ }^{4}$ traces were found in the remains of early civilizations

24 The second paragraph concludes that wheat

6 is of uncertain origin

7 is the largest grain crop

${ }^{8}$ originated in the Balkan States

${ }^{9}$ was first found in Asia 
Scientifically, butterflies and moths are grouped under the heading Lepidoptera, a term meaning "scale-winged." These insects have four broad wings covered with small, often brightly-colored scales.

Although they are grouped together, butterflies and moths are different in many ways. Butterflies usually fly during the day, while most moths do their flying at night. A butterfly has wiry antennae with knobs on the end. A moth, on the other hand, has feathery feelers. Butterflies usually molt into a chrysalis stage but do not spin cocoons. Moths, however, generally spin cocoons.

One member of Lepidoptera, the monarch butterfly, has reddish-brown wings with black veins and borders. It is famous for its migratory habit, traveling in the summer to places where milkweed grows. As winter approaches, the monarch must move to a warmer climate or die. It spends the winter, therefore, in coastal California, Florida, Texas, Mexico, and other places with a suitable climate.

During these migrations, many monarch butterflies swarm and cover groves of trees on the Monterey Peninsula in California. Every year they arrive in November, alight on the tall pines and cypresses, then leave around the middle of March.

The monarch may cause illness to an enemy which eats it. Animals, however, have learned to avoid it since the bright colors of the monarch are easily recognized. It is supposed that the viceroy butterfly is also avoided because its markings and coloring closely parallel the monarch.

The monarch lays its eggs singly on the undersides of milkweed leaves. In three to five days the egg hatchesnot into a beautiful butterfly-but into a caterpillar. During the caterpillar stage, it seems to do nothing but eat, grow, and molt. The new caterpillar gorges itself on the milkweed leaves, a behavior which has caused the monarch to be called the "milkweed butterfly." The caterpillar then splits its skin, or molts, four times. It attaches itself, head downward, on a thick, silken pad which it has spun on the lower surface of a leaf.

Next it splits its skin again, becoming a jade-green, transparent chrysalis which hangs on the leaf for ten days to two weeks while the adult butterfly is developing. The butterfly emerges and clings to the shell of the chrysalis for a brief period until its wings straighten out and harden. Then the adult butterfly is ready to fly. No longer interested in the milkweed leaf for food, the monarch flits from flower to flower searching for nectar. 
25 Scientifically, butterflies are called
1 caterpillars
2 Lepidoptera
3 monarchs
4 moths

26 Animals avoid the monarch butterfly because it

${ }^{6}$ can cause illness

' eats milkweed

${ }^{8}$ looks like the viceroy

${ }^{9}$ spins cocoons

27 Lepidoptera have four wings which are covered with

1 feathers

2 fur

3 fuzz

4 scales

28 The main purpose of the first two paragraphs is to

${ }^{6}$ compare butterflies and moths

7 describe the habits of butterflies

${ }^{8}$ explain how butterflies spin cocoons

9 emphasize the difference in moth and butterfly feelers

29 The time required for a monarch chrysalis to become a butterfly is

1 all summer

2 all winter

3 five to seven days

4 ten days to two weeks
30 The antennae of butterflies are

6 feathery

7 scaly

8 transparent

9 wiry

31 The last paragraph discusses the change from the

1. butterfly to the moth

${ }^{2}$ summer to the winter home

${ }^{3}$ chrysalis to the adult butterfly

4 cocoon to the caterpillar

32 A main food of the adult monarch butterfly is

6 moths

7 nectar

8 pine needles

9 viceroys

33 When they migrate, monarch butterflies often travel

1 great distances

2 in January

3 at night

4 with moths

34 All moths and butterflies are alike in that they

6 fly at night

${ }^{7}$ have scaly wings

${ }^{8}$ have wiry antennae

${ }^{9}$ spin cocoons 
MRS. BOWEN'S SIXTH GRADE CLASS

\begin{tabular}{|c|c|c|c|c|}
\hline \multirow{2}{*}{ PARTS } & \multicolumn{4}{|c|}{ CASTS } \\
\cline { 2 - 5 } & Cast 1 & Cast 2 & Cast 3 & Cast 4 \\
\hline Emil & Roy & Stanley & Carlos & Karim \\
\hline Renee & Barbara & Janiet & Maria & Elaine \\
\hline Geraldine & Chris & Carolyn & Kelly & Bonnio \\
\hline
\end{tabular}

Mrs. Bowen's sixth grade class is planning to put on a play. The characters in the play are named Emil, Renee, and Geraldine. The students in the class are reading through the play in groups called casts. The members of each cast and the parts they are reading are listed above.

Members of the same cast are called mates. Therefore, the mates of Janet are Stanley and Carolyn. A way to say this in symbols is:

$$
\mathrm{M}(\text { Janet })=[\text { Stanley, Carolyn }]
$$

This expression reads: The mates of Janet are Stanley and Carolyn.

Students who are reading the same part are called companions. For example, Roy, Stanley, Carlos, and Karim are all reading the part of Emil. Therefore, Roy's companions are Stanley, Carlos, and Karim. This can be expressed in symbols as:

$$
\mathrm{C}(\text { Roy })=[\text { Stanley, Carlos, Karim }]
$$

This expression reads: The companions of Roy are Stanley, Carlos, and Karim. 
35 Maria's cast is
${ }^{1}$ Cast 1
2 Cast 2
3 Cast 3
${ }^{4}$ Cast 4
${ }^{5}$ none of these

36 Kelly is reading the part of
6 Bonnie
7 Chris
8 Emil
9 Geraldine
${ }^{0}$ none of these

37 Roy and Stanley are
1 brothers
2 companions
3 cousins
4 mates
5 none of these

38 Which one of the following is not a student in Mrs. Bowen's class?
6 Barbara
7 Renee
${ }^{8}$ Roy
${ }^{9}$ Stanley
${ }^{0}$ none of these

39 How many companions does Kelly have?

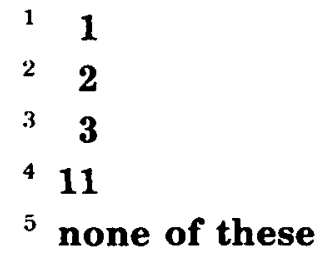

40 A companion of Carlos is
6 Carolyn
${ }^{7}$ Chris
8 Elaine
9 Maria
${ }^{0}$ none of these

41 A mate of Bonnie is
1 Carolyn
2 Karim
3 Kelly
4 Maria
${ }^{5}$ none of these

$42 \mathrm{M}$ (Barbara) $=$

${ }^{6}$ [Chris, Roy]

7 [Janet, Maria, Elaine]

8 [Renee]

${ }^{9}$ [Stanley, Janet, Carolyn]

${ }^{0}$ none of these 


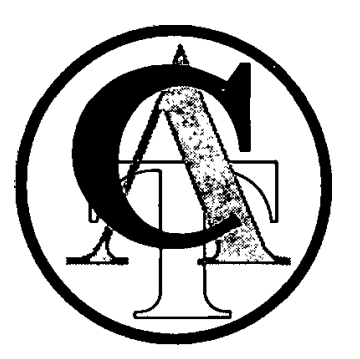

SECTION 3

\section{MATHEMATICS Computation}

purpose

directions
These items measure your ability to do basic mathematics operations.

This section contains 48 computation items. Work each item on scratch paper; then choose the answer you think is correct. If the correct answer is not given, choose "none of these." Fill in the space on your answer sheet that matches the letter of the answer you choose.

Read Sample Item A below.

$\begin{array}{lll}1 & \mathrm{C}^{4} \\ \mathrm{SAMPLE} \text { ITEM A } & { }^{\mathrm{D}} 5 \\ & \begin{array}{l}\mathrm{E} \text { none } \\ \text { of these }\end{array}\end{array}$

Look at the section of your answer sheet under the word "Computation" and see how the correct answer is marked. Answer space $\mathrm{E}$ is filled in for Sample Item A because " 2 ," the answer to 1 plus 1 , is not given.

Do Sample Item B. Fill in the space on your answer sheet that matches the letter of the answer you choose.

$\begin{array}{lrl} & 5 & \\ \text { SAMPLE ITEM B } & { }^{\mathrm{H}} \mathbf{3} \\ & & { }^{\mathrm{J}} \mathbf{9} \\ & & \mathrm{k} \text { none } \\ & & \text { of these }\end{array}$

You should have marked answer space $\mathrm{H}$ for Sample Item B because 5 minus 2 is " 3 ." 
Do Items 1-48 below and mark your answers on the answer sheet.

Be careful to keep your place on the answer sheet.

\section{ADDITION}

1

2

$\mathbf{3}$

\begin{tabular}{rrr} 
& A & 16 \\
& B & 96 \\
50 & C & 116 \\
+46 & D & 960 \\
\hline & E & $\begin{array}{l}\text { none } \\
\text { of these }\end{array}$ \\
& &
\end{tabular}

52

$+38$

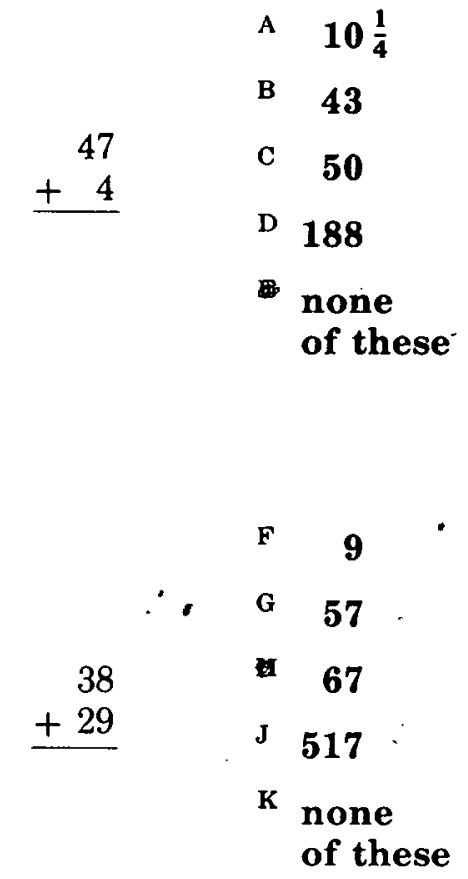

\section{SUBTRACTION}

5

$\begin{array}{lc}\text { A } & 34 \\ \text { - } & 44 \\ \text { C } & 48 \\ \text { D } & 108 \\ \text { E } & \text { none } \\ \text { of these }\end{array}$

$6 \$ 74.00-\$ 13.75=$

$\$ 60.25$

G $\$ 60.75$

H $\$ 87.25$

J $\$ 87.75$

${ }^{K}$ none of these

7

\begin{tabular}{rrr} 
& A & $\mathbf{2 5 1}$ \\
457 & & $\mathbf{3 5 1}$ \\
-106 & C & $\mathbf{5 6 3}$ \\
\hline & D & $\mathbf{3 , 5 0 1}$ \\
& E & $\begin{array}{l}\text { none } \\
\text { of these }\end{array}$ \\
& &
\end{tabular}

8

470

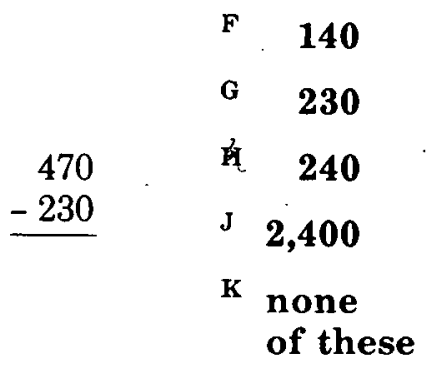

\section{ADDITION}

9

* 413

B 543

255

$+158$

C 633

D 653

$E$ none of these

10

$\begin{array}{rr}\text { F } & 28 \\ \text { G } & \mathbf{1 2 8}\end{array}$

308

$\mathbf{7 2 8}$

J 70,280

K none of these

11

A 15

B 22

50

$+28$

c 32

E 78

E none of these

12

F $\$ 38.80$

$\$ 26.32$

3.68

- $\$ 38.90$

.75

H $\$ 39.80$

$\begin{array}{r}8.15 \\ \hline\end{array}$

J $\$ 39.90$

$K$ none of these 


\section{SUBTRACTION}

13

\begin{aligned} & A 3,368 \\ \hline 5,806 & B 3,378 \\ $-2,438 &$ C 3,468 \\ \hline & D 3,478 \\ & E $\begin{array}{l}\text { none } \\ \text { of these }\end{array}\end{aligned}$

$14 \$ 45.00-\$ 14.25=$

F $\$ 14.70$

G $\$ 30.75$

H $\$ 31.75$

J $\$ 59.25$

$K$ none of these

15

\begin{tabular}{rll} 
& A & 41 \\
56 & B & 51 \\
-5 & C & 61 \\
\hline & D & 280 \\
& E & none \\
& of these
\end{tabular}

16

\begin{tabular}{rll} 
& F & $\$ 13.25$ \\
& G & $\$ 13.35$ \\
$\$ 15.00$ & H & $\$ 14.25$ \\
\hline$\quad 1.75$ & J & $\$ 14.35$ \\
& K & $\begin{array}{l}\text { none } \\
\text { of these }\end{array}$
\end{tabular}

ADDITION

17

\begin{aligned} & A 16,171 \\ 2,713 & B 17,763 \\ 4,689 & C 17,863 \\ 3,451 & D 18,874 \\ $+7,010 &$ E none \\ \hline & of these \end{aligned}

$18 \quad 3.5+6.25=$

F 6.55

G 6.60

H 9.25

J 9.75

$K$ none of these

19

$\$ 5.00+\$ .60+\$ 12.00+\$ 2.40=$
A $\$ \mathbf{\$ 8 . 1 2}$
B $\$ 19.00$
C $\$ 20.00$
D $\$ 31.70$
E none of these

$20 \quad 1$ yard 2 feet +2 yards 2 feet

F 3 yards 1 foot

G 3 yards 2 feet

4 yards

J 4 yards 1 foot

$K$ none of these

\section{SUBTRACTION}

21

A $\$ 34.51$

B $\$ 34.58$

$\$ 36.58$

- 2.03

C $\$ 36.51$

D $\$ 36.55$

E none of these

224 feet 5 inches -2 feet 10 inches

F. 1 foot 5 inches

G 1 foot 7 inches

$H 2$ feet 5 inches

J 2 feet 7 inches

$K$ none of these

$23 \quad 86.350-24.15=$
A 62.100
B 62.20
C 62.22
D 83.935
E none of these

$2492.260-21.14=$
F 70.012
G 70.12
H 71.102
J 71.12
$K$ none of these 


\section{MULTIPLICATION}

25

$\begin{array}{rll} & \text { A } & 236 \\ & \text { B } & 462 \\ 234 & \text { ब } & 468 \\ \times \quad 2 & \text { D } & 478 \\ & \text { E } & \begin{array}{l}\text { none } \\ \text { of these }\end{array} \\ & \end{array}$

26

$\begin{array}{rll} & \text { F } & 196 \\ & \text { G } & 204 \\ 200 \quad & \text { ' } & 600 \\ \times \quad 4 & \text { J } & 800 \\ & \text { K } & \text { none } \\ & \text { of these }\end{array}$

27

$\begin{array}{rcc} & \text { A } & 42 \\ & \text { B } & 44 \\ 51 & \text { C } & 58 \\ \times \quad 7 & & 357 \\ & & \text { E } \\ & \text { none } \\ & \text { of these }\end{array}$

28

\begin{tabular}{rrr} 
& F & 796 \\
& G & 814 \\
805 & & 7,245 \\
$\times \quad 9$ & & \\
\hline & J & 72,045 \\
& K $\begin{array}{l}\text { none } \\
\text { of these }\end{array}$
\end{tabular}

\section{DIVISION}

29

30

$6 \longdiv { 1 2 }$

A $\quad \frac{1}{2}$
- $\quad 2$

C 6

D 72

E none of these

31

$9 \longdiv { 4 5 }$

a 5

H 6

J 9

K none of these

32

$7 \longdiv { 7 0 }$

$\begin{array}{lc} & \frac{1}{10} \\ \text { B } & 1 \\ \text { C } & 7 \\ & 10 \\ \text { E none } & \text { nof these }\end{array}$

$3 0 \longdiv { 2 1 0 }$

F 7

G 70

H 180

J 700

$K$ none of these
35

34

$\begin{array}{r}479 \\ \times \quad 32 \\ \hline\end{array}$

$\begin{array}{r}42 \\ \hline\end{array}$

$\begin{array}{r}489 \\ \times \quad 40 \\ \hline\end{array}$

$\begin{array}{r}40 \\ \hline\end{array}$

400

$\times 200$

A $\quad 511$

B 5,382

ค․ 15,328

D $\mathbf{1 7 , 4 1 8}$

$E$ none of these

F $\quad 529$

G $\quad \mathbf{1 , 9 5 6}$

It 18,460

J 19,560

$K$ none of these

A $\mathbf{8 0 0}$

B 6,000

C 8,000

- 80,000

E none of these

36

F $\quad \mathbf{4 9 , 0 8 8}$

G $\quad 57,008$

2,036

208
$\times$

$\begin{array}{r}413,488 \\ \hline\end{array}$

J. 423,488

none of these 
DIVISION

37

$2 \longdiv { 5 \text { yards } 1 \text { foot } }$
A 2 yards
- 2 yards 1 foot
C 2 yards $5 \frac{1}{2}$ feet
D 3 yards
$E$ none of these

38

$$
\begin{array}{rr}
\text { F } & 29 \\
\text { G } & 209
\end{array}
$$

$3 6 \longdiv { 7 , 5 2 4 }$

$\begin{array}{ll}\text { H } & 210 \\ \text { J } & 219 \\ \text { K } & \text { none } \\ \text { of these }\end{array}$

39
A 16
B 101
C 106
D 160
E none of these

$4 \longdiv { 4 2 4 }$

40

$\begin{array}{llll} & & \text { F } & \mathbf{6 0} \\ & \text { G } & \mathbf{6 1} \\ & & \text { H } & \mathbf{6 6} \\ & \text { J } & \mathbf{3 6 0} \\ & \text { K } & \text { none } \\ & & \text { of these }\end{array}$

\section{MULTIPLICATION}

415 feet 6 inches $\times \quad 3$
A 5 feet 9 inches
B 6 feet 6 inches
C 16 feet 6 inches
D 16 feet 8 inches
$E$ none of these

42

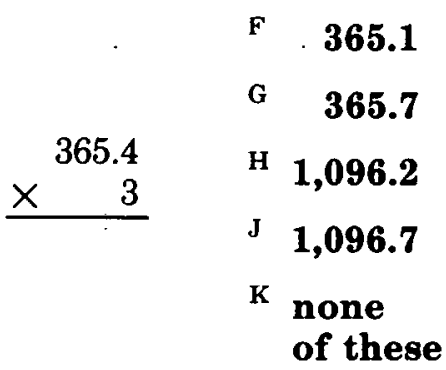

43

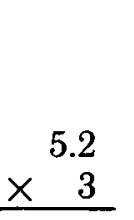

44

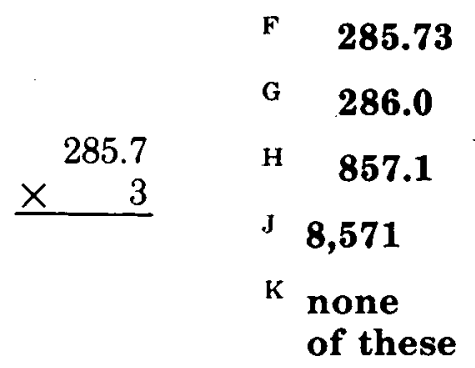

A $\quad \mathbf{1 5 6}$
DIVISION

45

A 9 R 32

B $\quad 9 \frac{25}{27}$

$5 4 \longdiv { 4 , 8 9 2 }$ C $89 \frac{6}{54}$

D $90 \frac{16}{27}$

E none of these

\begin{tabular}{|c|c|c|c|}
\hline & & F & 2 \\
\hline & & G & 20 \\
\hline$4 0 0 \longdiv { }$ & 8,000 & $\mathbf{H}$ & 200 \\
\hline & & $\mathrm{J}$ & 8,400 \\
\hline & & $\mathbf{K}$ & $\begin{array}{l}\text { none } \\
\text { of thes }\end{array}$ \\
\hline
\end{tabular}

46

47

A $\quad 1 \frac{1}{10}$

B 11

$4 0 \longdiv { 4 4 0 }$

C .110

D 1,100

E none of these

48

F $\quad \mathbf{1 2 6}$

G $\quad \mathbf{1 . 2 6}$

$4 \longdiv { 5 . 0 4 } \quad$ H $\mathbf{1 2 . 6}$

J 126

$\mathrm{K}$ none of these 


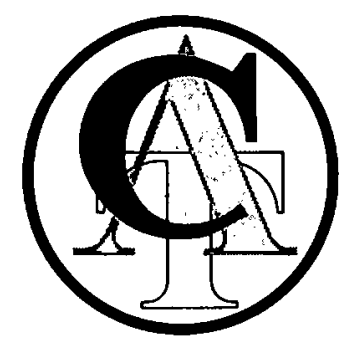

SECTION 4

MATHEMATICS Concepts

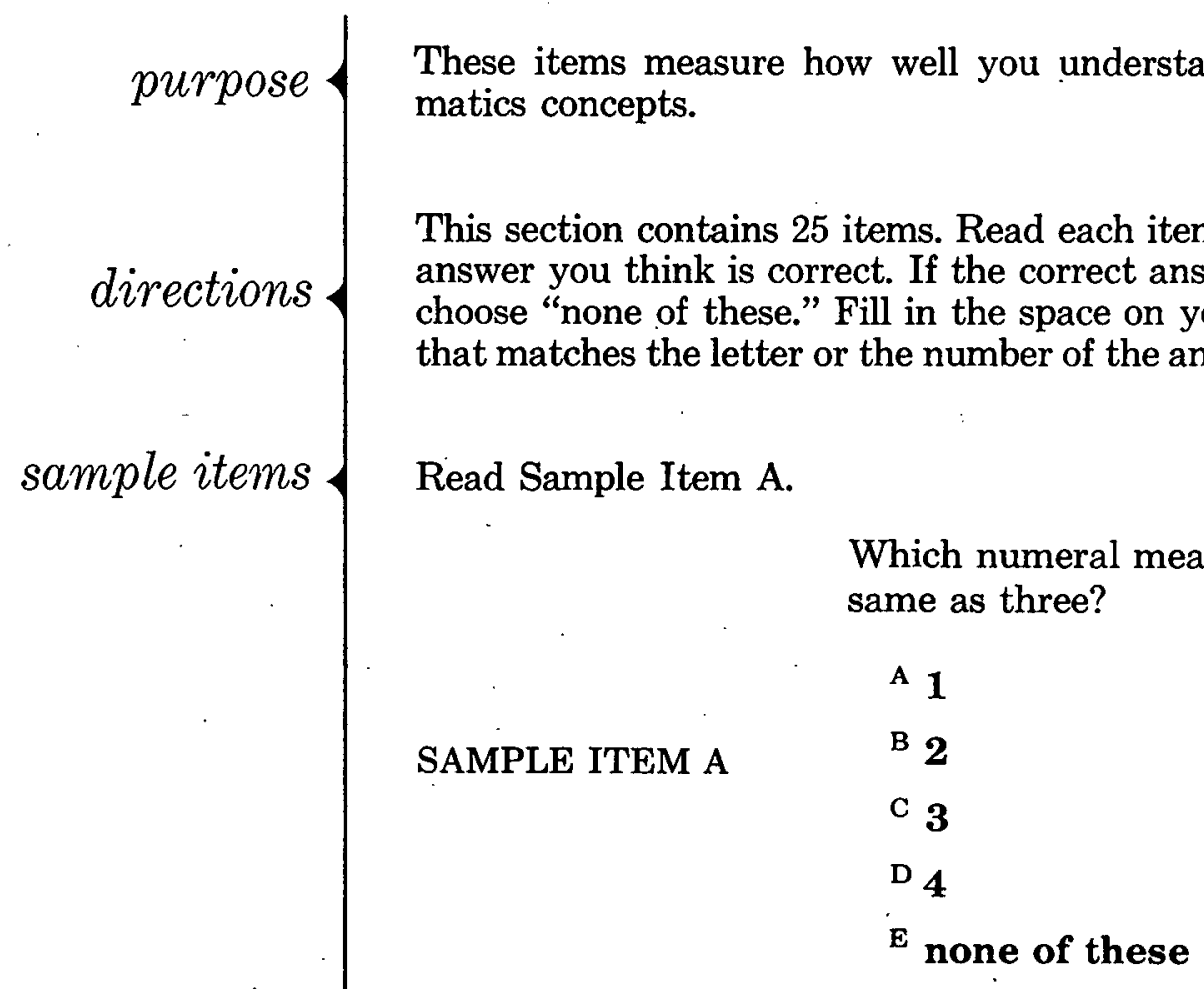

Look at the section of your answer sheet under the word "Concepts" and see how the correct answer is marked. Answer space $\mathrm{C}$ is filled in for Sample Item A because three means the same as " 3. "

Do Sample Item B. Fill in the space on your answer sheet that matches the letter of the answer you choose.

Which number belongs in the blank?

$\begin{array}{llll}1 & 2 & 3 & 4\end{array}-$
${ }^{\mathrm{F}} \mathbf{1}$
${ }^{\mathrm{G}} 3$
${ }^{\mathrm{H}} 6$
${ }_{\mathrm{J}} 7$
${ }_{\mathrm{K}}$

You should have marked answer space $\mathrm{K}$ for Sample Item B because " 5 ," the next number in the sequence, is not given. 
Do Items 1-25 below and mark your answers on the answer sheet.

Be careful to keep your place on the answer sheet.

1 Which figure below has the largest area?
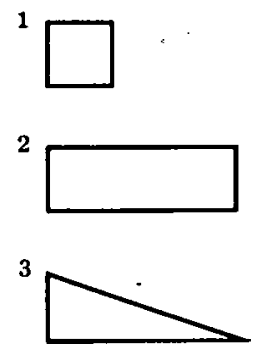

4
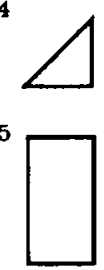

2 What speed does this speedometer show?

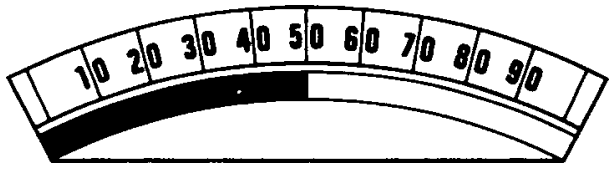
A 40 miles per hour
B 50 miles per hour
C 55 miles per hour
D 58 miles per hour
$E$ none of these

3 What number belongs in the box?

$$
\begin{array}{ll} 
& 5 \times 8=\square \\
\text { F } & \frac{5}{8} \\
\text { G } & 1 \frac{3}{\overline{5}} \\
\text { H } & 13 \\
\text { J } & 40 \\
\text { K none of these }
\end{array}
$$

4 Which of the following numerals names the largest number?
A 209
B 220
C 198
D 213
E 218

5 How much money is three quarters and two nickels?
F $\quad \mathbf{\$ . 2 5}$
G $\$ \mathbf{\$ . 7 5}$
H $\mathbf{\$ . 8 5}$
J $\$ 1.25$
$K$ none of these

6 What fraction of the rectangle shown is shaded?

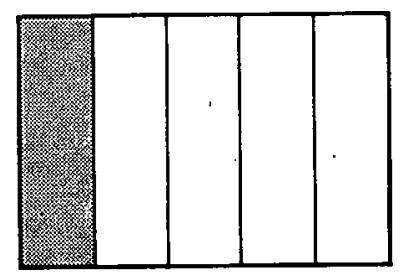
A $\frac{1}{6}$
B $\frac{1}{5}$
C 1
D 1
E none of these 
7 Which of the following numerals names the smallest number?

F 321

G 226

H. 128

J 190

K 209

8 Which one of the following symbols belongs in both boxes?

$3 \square 1=1 \square 3$
A +
B -
$\mathrm{C}+$
D $\sqrt{ }$
E $\triangle$

9 means

1 dime

2 dollar

${ }^{3}$ nickel

4 quarter

${ }^{5}$ none of these

10 What numeral belongs in the box?

$$
5 \times 2=\square \times 5
$$
A 2
B $\cdot \mathbf{5}$
C 7
D 10
E none of these

11 In the figure below, what is the name of the longest side?

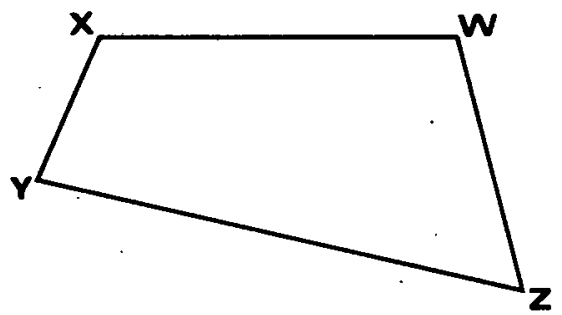
$1 \mathbf{X Y}$
2 YZ
${ }^{3} \mathbf{Z W}$
4 WX
5 WY

12 The sides of the triangle below each measure 6 feet in length. What is the distance around the triangle?

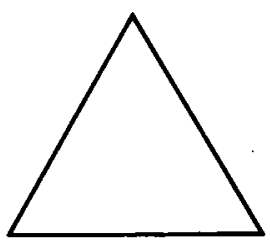
A 6 feet
B 12 feet
C 18 feet
D 24 feet
$E$ none of these

13 Which of the following means the same as lb.?
1 ounce
2 pi
${ }^{3}$ pound
4 yard
5 none of these 
14 Which digit in the numeral 487,023 is in the hundreds' place?
A 0
B 2
C 4
D 7
E none of these

15 Which of the following figures has only three angles?

1 rectangle

2 square

3 trapezoid

4 triangle

5 none of these

16 Which of the following means the same as one hundred eleven?
A $\quad \mathbf{1 , 0 1 1}$
B $\quad 1,101$
C 10,011
D 100,011
E none of these

17 Which of the following means the same as four dollars and four cents?
F \$4.4c
G $\$ 4.4$
H $\$ 4.04$
J. $\$ 4.44$
$K$ none of these

18 Which of the following has more than four sides?

1 pentagon

- 2 rectangle

3 square

4 triangle

5 none of these

19 Which of the following means the same as three thousand five?
A $\quad 305$
B $\quad 3,005$
C $\quad 3,500$
D $3,000,005$
${ }^{E}$ none of these

20 \% means
1 degree
2 inch
${ }^{3}$ per cent
4 ratio
${ }^{5}$ none of these

21 Which of the following means the same as $\frac{25}{3}$ ?
A $8 \frac{1}{3}$
B $8 \frac{2}{3}$
C $9 \frac{1}{3}$
D $9 \frac{2}{3}$
$E$ none of these 
22 The Roman numeral $\mathrm{C}$ means the same as
F 100
G 200
H 300
J $\mathbf{5 0 0}$
$\mathrm{K}$ none of these

232 hours 105 minutes $=$
A 3 hours 5 minutes
B 3 hours 45 minutes
C 3 hours 55 minutes
D 4 hours 5 minutes
E none of these

24 In the numeral 426.1, which digit is in the hundreds' place?

$$
\begin{array}{ll}
\text { F } & 1 \\
\text { G } & 2 \\
\text { H } & 4 \\
\text { J } & 6 \\
\text { K } & \text { none of these }
\end{array}
$$

25 Which of the following means the same as forty dollars and six cents?
A $\$ 40.64$
B $\$ 40.6$
C $\mathbf{4 0 . 0 6}$
D' $\$ 40.06$
E none of these 



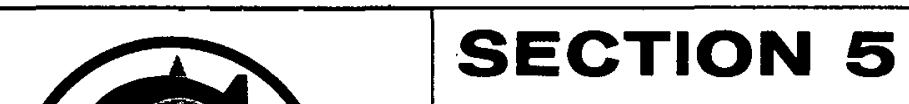

MATHEMATICS Problems

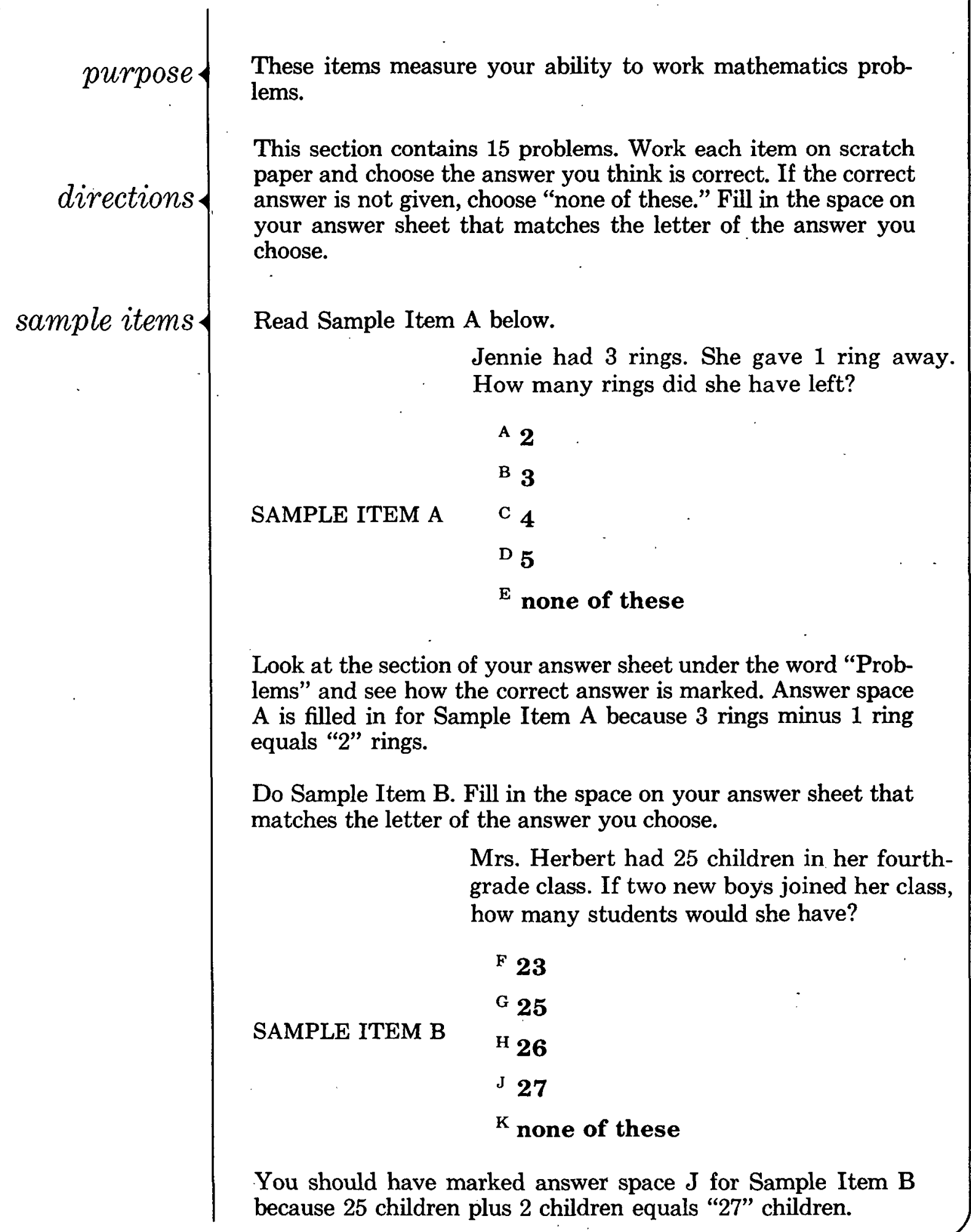


Do Items 1-15 below and mark your answers on the answer sheet.

Be careful to keep your place on the answer sheet.

1 Leslie has 5 white chickens and 8 brown chickens. How many chickens does she have in all?
A 3
B $\mathbf{5}$
c 8
1. 13
E none of these

2 A rancher had 15 cows. He sold 5 of them. How many cows did he have left?
F 9
G 16
H 21
J 80
4 none of these

3 Leon had 12 pieces of candy. He gave 3 pieces to Lauren and 5 pieces to Jorge. How many did he have left?
A 2
1. 4
C 7
D 9
$E$ none of these

4 Earl has 4 model airplanes. Klàus has 2 times as many. How many model airplanes does Klaus hàve?

$$
\begin{array}{lc}
F & 2 \\
\text { G } & 6 \\
\text { J } & 8 \\
J & 16 \\
\text { K none of these }
\end{array}
$$

$5 \quad$ Ted solved 4 problems. Sam solved 3 times as many. How many problems did Sam solve?
A 3
B 5
C 7
D 12
E none of these

6 The Oak Street School Chess Club had 6 members. Three of the members quit and then 1 new student joined. How many members were finally in the club?

$$
\begin{array}{ll}
\text { F } & 2 \\
\text { \$ } & 4 \\
\text { H } & 8 \\
\text { J } & 10 \\
\text { K } & \text { none of these }
\end{array}
$$


7 James raised 40 rabbits and sold 24 of them. He divided those remaining evenly among 4 friends. How many rabbits did each receive?
4.4
B 8
C 10
D 16
E none of these

8 Katie spent 42 cents on pencils. Each pencil cost 6 cents. How many pencils did she buy?

$$
\begin{array}{ll}
\text { F } & 3 \\
\text { G } & 6 \\
\text { K } & 7 \\
\text { J } & 36 \\
\text { K } & \text { none of these }
\end{array}
$$

9 Ethel had $\$ 5.00$. She paid 35 cents for a ribbon, $\$ 1.65$ for a new book, and 50 cents for lunch. Then she spent the amount she had left for Christmas gifts. How much did the Christmas gifts cost?
A $\$ 2.00$
B $\$ 2.50$
C $\$ \mathbf{\$ 3 . 0 0}$
D $\$ 5.00$
Tone of these

10 A room had 6 rows of desks with 7 desks in each row. Five desks were removed from the room. How many desks remained?

$$
\begin{array}{ll}
F & 8 \\
\text { G } & 13 \\
\times & 37 \\
\text { J } & 42 \\
\text { K } & \text { none of these }
\end{array}
$$

11 How many square yards are there in a rug which is 3 yards wide and 4 yards long?
A 6
B 7
C 9
12
$E$ none of these

12 On a map, 1 inch represents 80 miles. The distance between two cities on the map is 2 inches. How many miles apart are the two cities?

$$
\begin{array}{ll}
\text { F } & 40 \\
\text { G } & 100 \\
& 120 \\
J & 160 \\
\text { K none of these }
\end{array}
$$


13 For a Thanksgiving party, two thirds of a class brought food. If there were 30 students in the class, how many students brought food?
A 10
B 15
\& 20
D 27
$E$ none of these

15 Jack weighs 95 pounds, Peter weighs 75 pounds, and Robert weighs 100 pounds. What is their average weight in pounds?
A 85 pounds
B $87 \frac{1}{2}$ pounds
C 90 pounds
D $92 \frac{1}{2}$ pounds
none of these

14 Theresa had 12 cookies. She divided them equally among 3 other girls and herself. How many cookies did each girl receive?

$$
\begin{array}{lr}
\text { K } & 3 \\
\text { G } & 4 \\
\text { H } & 9 \\
\text { J } & 11 \\
\text { K } & \text { none of these }
\end{array}
$$




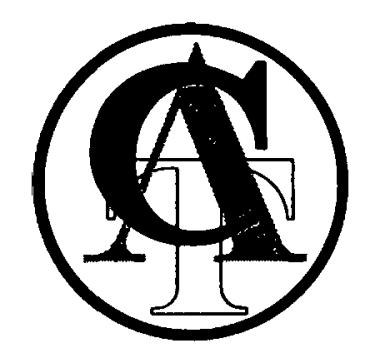

SECTION 6

\section{MATHEMATICS Fractions}

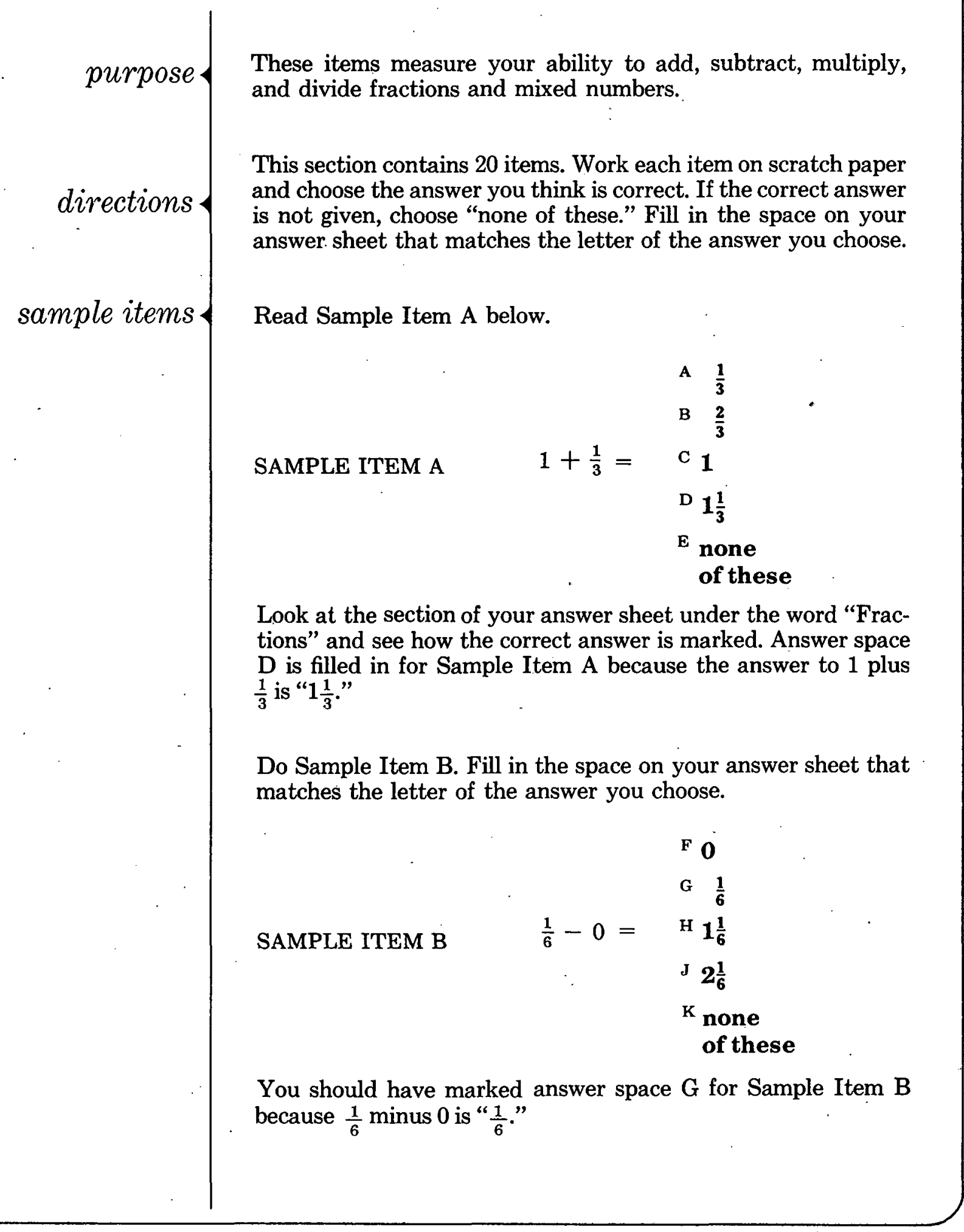


Do Items 1-20 below and mark your answers on the answer sheet.

Be careful to keep your place on the answer sheet.

1

2

3
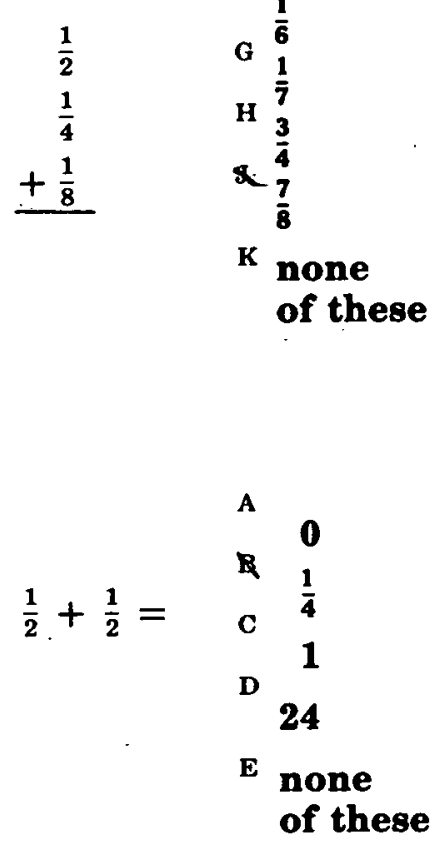

4

$$
\begin{array}{rr} 
& F \\
21 \frac{1}{3} & \text { G } \begin{array}{l}
25 \frac{1}{12} \\
25 \frac{1}{7} \\
+4 \frac{1}{4}
\end{array} \quad \text { H } \begin{array}{l}
25 \\
7 \\
25 \frac{7}{12}
\end{array} \\
\hline & \text { Kone } \\
\text { nof these } \\
\text { of the }
\end{array}
$$

5

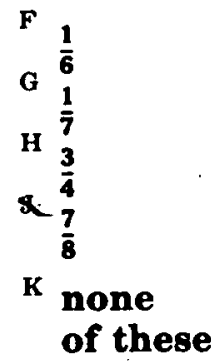

of these

8

6

7

A $3 \frac{5}{8}$
B $3 \frac{1}{2}$
C $3 \frac{3}{4}$
D $3 \frac{7}{8}$
N none
of these

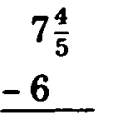

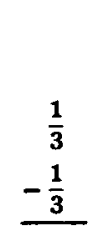

$\begin{array}{r}\frac{2}{3} \\ +3 \frac{1}{6} \\ \hline\end{array}$

$-6$

8

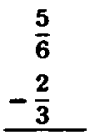

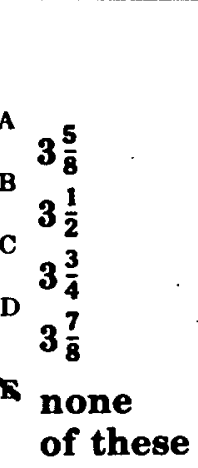

$\begin{array}{lr}\text { F } & 4 \\ \text { G } & \mathbf{5} \\ \text { N } & 1 \frac{1}{5} \\ \text { J } & 1 \frac{4}{5}\end{array}$

$13 \frac{4}{5}$

$k$ none of these

4

$\begin{array}{ll}\text { B } & 0 \\ \text { C } & \frac{1}{9} \\ & \frac{1}{6} \\ 2 & \frac{2}{3} \\ \text { E } & \begin{array}{l}\text { none } \\ \text { of these }\end{array}\end{array}$

T.

G $\frac{1}{6}$

H $\begin{aligned} & \overline{3} \\ & \mathbf{5}\end{aligned}$

J $\begin{gathered}\overline{6} \\ 6\end{gathered}$

K none of these
9

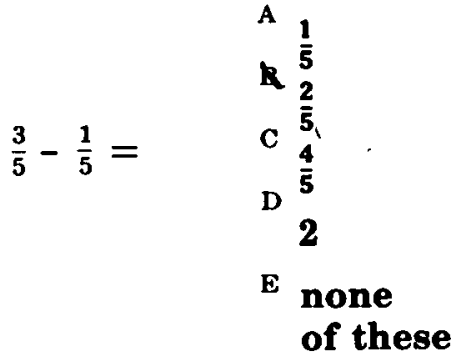

10

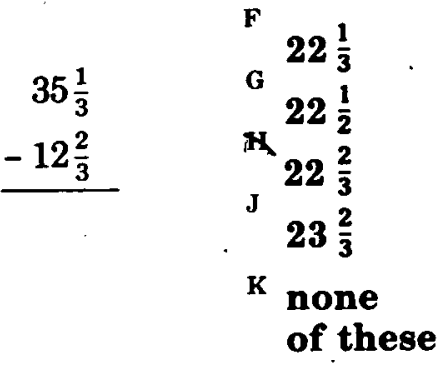

11

$$
4 \times \frac{1}{2}=\begin{array}{ll}
\text { A } & \frac{1}{8} \\
& \underbrace{\frac{1}{2}} \\
& 2 \\
& 4 \frac{1}{2} \\
\text { E none } \\
\text { of these }
\end{array}
$$

12

$$
\begin{array}{ll}
\mid \begin{array}{l}
\mid \\
\text { G }
\end{array} & \frac{1}{4} \\
\text { H } & \frac{3}{4} \\
& 1 \\
\text { J } & 1 \frac{1}{2} \\
\text { K none } \\
\text { of these }
\end{array}
$$


13

$$
\begin{array}{ll}
\frac{1}{4} \times \frac{4}{5}=\quad & \text { A } \frac{1}{5} \\
& \text { C } \frac{1}{4} \\
& \text { D } \frac{3}{10} \\
& \text { E none } \\
& \text { of these }
\end{array}
$$

14

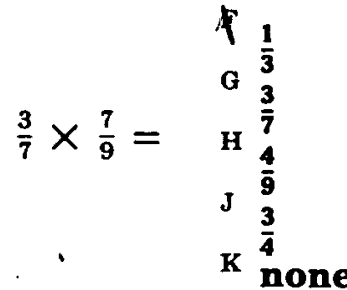

$$
\begin{aligned}
& \text { of these }
\end{aligned}
$$

15

$$
\begin{aligned}
& \begin{array}{rr}
\text { A } & \frac{3}{4} \\
\text { B } & 1 \frac{1}{6}
\end{array} \\
& 6 \times 4 \frac{1}{2}=\quad 10 \frac{1}{2} \\
& \text { D } 27 \\
& E \text { none }
\end{aligned}
$$

16

$$
\begin{aligned}
& \frac{3}{5} \div \frac{3}{5}=\text { G }^{\frac{9}{25}} \\
& \text { J } 2 \frac{7}{25} \\
& K \text { none }
\end{aligned}
$$

17

$$
\begin{aligned}
& \frac{1}{2} \div \frac{1}{4}=\quad \begin{array}{cc}
{ }^{A} & \frac{1}{8} \\
& \frac{1}{4} \\
& \\
& \frac{3}{2} \\
2
\end{array} \\
& E \text { none }
\end{aligned}
$$

18

$$
\begin{aligned}
& 2 \div \frac{1}{2}={ }_{\text {J }^{4}} \begin{array}{ll}
\text { H } & \frac{1}{4} \\
2
\end{array} \\
& K \text { none }
\end{aligned}
$$

19

$$
\begin{array}{lll}
\frac{3}{4} \div \frac{1}{2}= & \text { A } & \frac{1}{8} \\
& \frac{1}{3} \\
\text { C } & \frac{3}{8} \\
& \text { E } & 1 \frac{1}{2} \\
& \text { Eone } & \text { of these }
\end{array}
$$

20

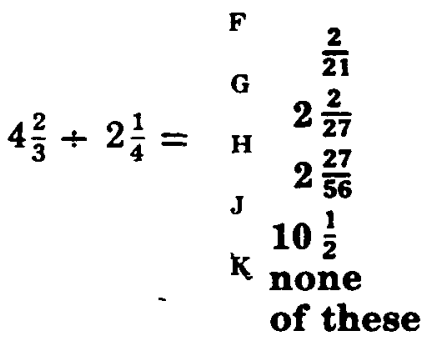




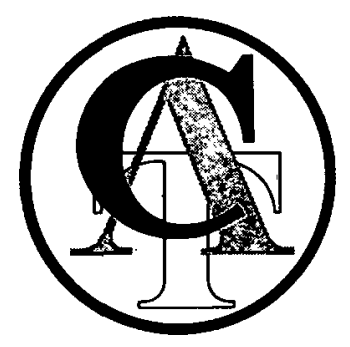

\section{SECTION 7}

\section{LANGUAGE Capitalization}

directions

sample items
These items measure how well you use capital letters in written expression.

This section contains a story with 38 capitalization items. Only the lines with numbers beside them are items. Lines that are not numbered are not items. Each item is only one line long and has no more than five words. Each of the words in an item is an answer choice. Some items have an error in capitalization, but no item has more than one error. Decide which word, if any, needs to be capitalized in each item. Count from the left to determine whether it is the first, second, third, fourth, or fifth word in the line. Fill in the space on your answer sheet that matches the number of the word you choose. If no word in an item needs capitalization, fill in answer space $\mathrm{N}$ for "None."

Read the sample passage below.

\section{Although it was very foggy,}
A Rachel and i wanted to go out in the yard
B and play with my dog.

Look at the section of your answer sheet under the word "Capitalization" and see how the correct answer for Sample Item A is marked. Answer space 3 is filled in because the pronoun " $i$," the third word in the line, should be capitalized.

Do Sample Item B. Fill in the space on your answer sheet that matches the number of the word that needs to be capitalized.

You should have marked answer space $\mathrm{N}$ for Sample Item $\mathrm{B}$ because no word in the line needs to be capitalized. 
Do Items 1-38 below and mark your answers on the answer sheet.

Be careful to keep your place on the answer sheet.

1 Before the christmas vacation, Mrs.

2 ching advised her class to see New York City during

3 the holidays. The following monday

4 dr. Garcia took his sons

Juan and Carlos on a tour.

First they rode the subway

5 to the Empire state Building

6 on Fifth avenue. They took the elevator to the roof. when they got to the top, the boys jumped out and ran to the protective

railing. the whole city appeared below them.

Next they went to the United nations. A pretty, young english girl guided them through the building. after the tour, they attended a brief session of the General Assembly. when it was over, Juan said, "The ambassador from israel reminded me of old uncle Salvador."
17 18 19 20
Eiko and kelly go to school in seaside, California. One day miss Waller asked them to do a science project about life in the pacific Ocean tidepools. she told them both, "first you will have to read the Monterey journal to see when the tides are low. Then $\mathrm{i}$ want you to get dr. Hedgepeth's book that's called introduction to Seashore Life. you should take it with you whenever you go out." "Oh boy!" said Eiko, "i feel just like an explorer. we may discover a new plant or animal."

That Saturday they took the Pico avenue bus down to Otter Beach. Eiko said, "Let's find at least 30 different species." "You must be kidding," her friend said. "Remember the poem we read yesterday:

be not impressed by quantity; always aim first at quality." 


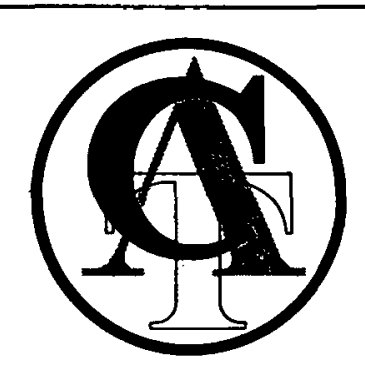

SECTION 8

LANGUAGE Punctuation $I$ and II

directions

sample items
These items measure how well you understand punctuation.

This section consists of two parts: Punctuation I and Punctuation II. The first part contains a letter with 30 items. The second part contains a story with 12 items.

In Punctuation I each line of the letter is numbered and is an item. Some items have an error in punctuation, but no item contains more than one error. In each item, decide which, if any, punctuation mark has been left out. Fill in the space on your answer sheet that shows the missing punctuation mark. If no punctuation mark is missing, fill in answer space $\mathrm{N}$ for "None."

Look at the section of your answer sheet under the words "Punctuation I." Note that the answer choices are arranged in a column underneath the item number. In this section, the item numbers go across the page, left to right, instead of down the page.

Read the sample passage below.

A $\mid \begin{gathered}\text { May 18,1970 } \\ \text { Dear Gene } \\ \text { When are you coming to. } \\ \text { visit me Mother said you } \\ \text { could stay the whole summer. } \\ \text { Your friend, }\end{gathered}$

Look at the Punctuation I section of your answer sheet and see how the correct answer for Sample Item $A$ is marked. In the answer column for Sample Item A, the space in the comma row is filled in because a comma belongs after "Gene."

Do Sample Item B. Fill in the space on your answer sheet that shows the missing punctuation mark.

You should have filled in the answer space for the question mark because "When are you coming to visit me" is a question. 
Do Items 1-30 below and mark your answers on the answer sheet.

Be careful to keep your place on the answer sheet.

\begin{tabular}{|c|c|}
\hline 1 & 65 Oak St \\
\hline 2 & Oak Grove Illinois \\
\hline 3 & January 51970 \\
\hline 4 & Dear Kaz \\
\hline 5 & How was your Christmas Mine \\
\hline 6 & was great I got a \\
\hline 7 & brand new baseball a bat, \\
\hline 8 & and a cap. Tonys present \\
\hline 9 & to me, however, was really \\
\hline 10 & my favorite Can you guess \\
\hline 11 & what it was Surprise, it \\
\hline 12 & was a snail When I \\
\hline 13 & first saw it it didn't \\
\hline 14 & move. I was really worried. \\
\hline 15 & But Mom said "He's asleep." \\
\hline 16 & I put him in water \\
\hline 17 & and watched. Very slowly B.J \\
\hline
\end{tabular}

18

19

20

21

22

23

24

25

26

27

28

29

30 (thats what I named him)

began to poke around $\mathrm{My}$

baby sister shouted, He's moving, he's moving!" And he was.

Friday Dec. 26, we built him a home of a plastic bowl dirt, and rocks.

Theres a screen on top.

Well I have to stop so that I can read my new book on snails Lets get together very soon.

Your friend

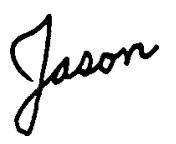




\section{directions}

\section{LANGUAGE Punctuation II}

Punctuation II contains a story with 12 items. Only the lines with numbers beside them are items. Lines that are not numbered are not items. Each item is only one line long.

Look at the section of your answer sheet under the words "Punctuation II." Note that the answer choices for each item are set in one column with an upper and lower part.

In each item, decide which punctuation mark, if any, is missing. Fill in the space in the upper part of the column that shows the missing punctuation mark. Now decide where the missing mark belongs. If this mark were placed in the item, it would appear closest to one word. Find that word. Then count from the left to determine the number of that word. Each word, abbreviation, initial, or number group in an item is an answer choice. Mark the place in the lower part of the column that shows the number of the answer you choose. If no punctuation mark is missing, fill in answer space $\mathrm{N}$ for "None" in the upper part of the column. Then go on to the next item. No item has more than one error.

Read the sample passage below.
\begin{tabular}{l|}
$\mathbf{A}$ \\
$\mathbf{B}$
\end{tabular}
I wrote a paper on
Columbus Ohio, my favorite city.
Have you ever been there

Look at the section of your answer sheet for Punctuation II and see how the correct answers are marked. In the upper part of the column, the space in the comma row is filled in for Sample Item A because a comma is missing. In the lower part of the column, answer space 1 is filled in because "Columbus," the first word in the item, is closest to the missing comma.

Do Sample Item B. Fill in the space in the upper part of the column and the space in the lower part of the column for the answers you choose.

In the upper part of the column, you should have filled in the answer space for the question mark because "Have you ever been there" is a question. In the lower part of the column, you should have filled in answer space 5 because "there," the fifth word in the item, is nearest to the question mark. 
LANGUAGE Punctuation / and //

40

Do Items 1-12 below and mark your answers on the answer sheet.

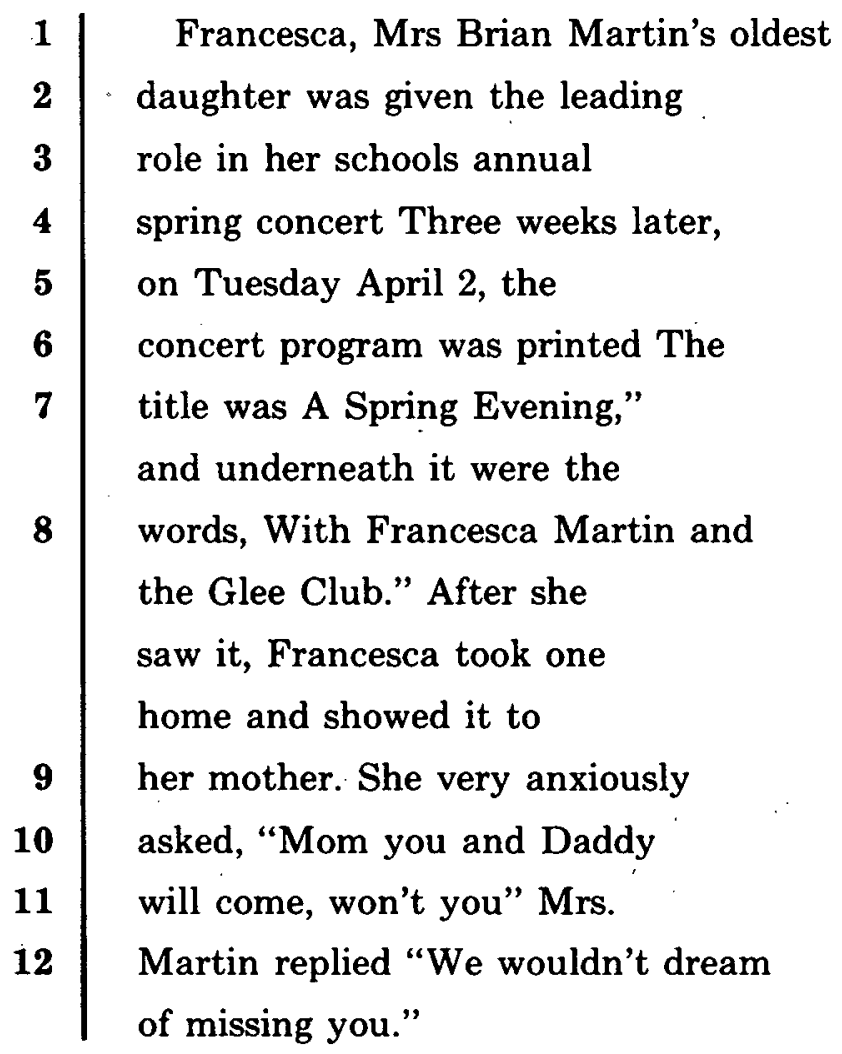




\section{SECTION 9}

\section{LANGUAGE Usage and Structure}

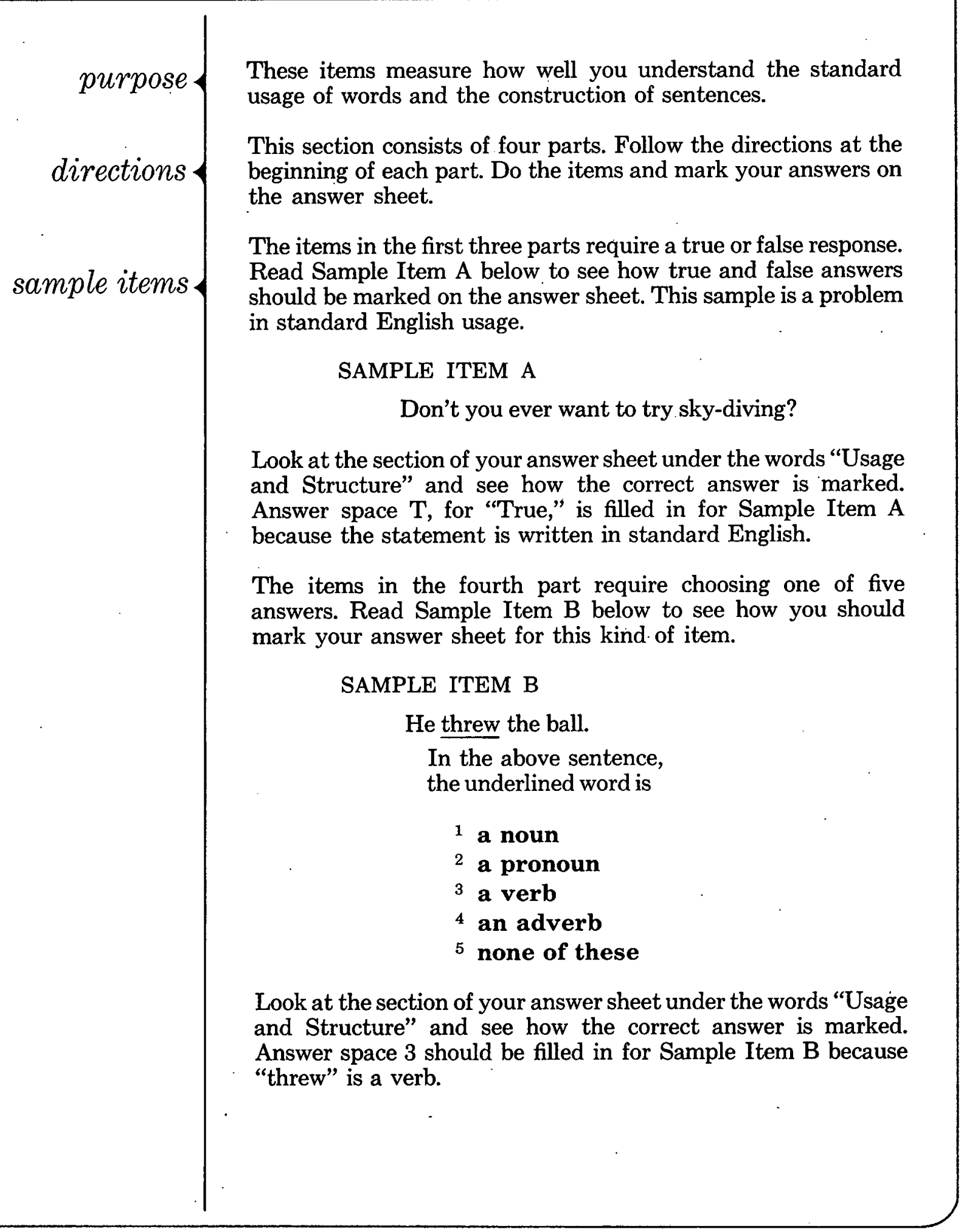


In Items 1-24 below, decide if each statement is written in standard English. If the statement is in standard English, fill in answer space $T$ on your answer sheet. If it is not in standard English, fill in answer space F. Be careful to keep your place on the answer sheet.

1 Emilia and her are going to hike in the hill country around Kerrville.

2 Bernie runs faster than any other boy on his team.

3 Is she the one who has the German shepherd?

4 Mr. Carreon came to our class yesterday and spoke about Mexican art.

5 Baseball games is fun to watch.

6 The men's uniforms were dirty and torn after the game.

7 Let Nellie read by herself.

8 Mrs. Tam arranges flowers very well.

9 The storm winds blew the fishing boat miles off course.

10 Are we the people you were looking for?

11 The maple leaves have fallen already.

12 The dog shook himself and got water all over me.
13 Those rocket books are my favorites.

14 My two goldfish don't need any food today.

15 The plumber done a good job.

16 The sparrow was so small that I couldn't hardly see it.

17 Stella divided the cards between Hal and me.

18 The woodpecker had flew from the rooftop to the oak tree.

19 One of those books don't belong here.

20 Our favorite mountain lake must be froze by now.

21 Foursquare is her favorite game, but she plays bad.

22 Nick should have went when he had the chance.

23 Louis has rode every bus in Tuscaloosa.

24 Peeling a onion always makes her cry. 
In Items 25-29 below, decide if each statement is a complete sentence. If it is a complete sentence, fill in answer space $T$ on your answer sheet. If it is not a complete sentence, fill in answer space $F$.
In Items 30-34 below, decide if the original sentence is transformed correctly. If it is transformed correctly, fill in answer space $T$ on your answer sheet. If it is not transformed correctly, fill in answer space $F$.
25 Her aunt baked some pecan pies.

26 Day by day the spring flowers bloomed and died.

27 Calling to his dog and running at top speed after his friends.

28 The family of deer paused quietly under the big oak tree at the edge of the clearing.

29 The little boy with the freckles and the carrot-colored hair.
30 She was at school last Saturday.

The statement above can be transformed into a question which asks, "Where is she?"

31 Kathleen is talking with her teacher.

The statement above can be transformed into a negative statement which reads, "Kathleen is talking not with her teacher."

32 Carmen stayed in her bed all day.

The statement above can be transformed into a question which asks, "When is Carmen going to stay in her bed?"

33 Derek will never finish the story.

The statement above can be transformed into an affirmative statement which reads, "Derek can always finish the story."

34 They have grown a vegetable garden.

The statement above can be transformed into a question which asks, "Are they growing a vegetable garden?" 
Read Items 35-41 and choose the best answer. Mark your answers on the answer sheet.

35 Maura's ribbons are very

In the above sentence, the blank must be filled with

1 a conjunction

2 a determiner

3 a noun

4 an adjective

5 none of these

36 Two hawks were slowly circling above Maidu Peak.

The part underlined above is

6 a conjunction

7 a prepositional phrase

8 a relative clause

9 an appositive

0 none of these

37 The setting sun looks

In the above sentence, the blank must be filled with

1 a conjunction

2 a preposition

3 an adjective

+ an adverb

5 none of these

38 The mother chimpanzee gently held her baby in her arms.

The part underlined above is

6 a conjunction

7 a prepositional phrase

8 a relative clause

9 an appositive

${ }^{0}$ none of these
39 The past inflectional morpheme ending -ed is added to

1 adjectives

2 adverbs

3 nouns

4 verbs

5 none of these

40 Axel easily won the race.

In the above sentence, the underlined word is

6 a noun

7 a verb

8 an adjective

9 an adverb

${ }^{0}$ none of these

41 The team that has the ball is likely to win.

The part underlined above is

1 a conjunction

2 a prepositional phrase

3 a relative clause

4 an appositive

5 none of these 


\section{SECTION 10}

LANGUAGE Spelling

Read Sample Item A below.

$$
\begin{aligned}
& \text { SAMPLE ITEM A } \\
& { }^{1} \text { pichure } \\
& { }^{2} \text { rose } \\
& { }^{3} \text { teacher } \\
& { }^{4} \text { yellow } \\
& { }^{5} \text { none of these }
\end{aligned}
$$

Look at the section of your answer sheet under the word "Spelling" and see how the correct answer is marked. Answer space 1 is filled in for Sample Item A because "pichure" is the misspelled word.

Do Sample Item B. Fill in the space on your answer sheet that matches the number of the answer you choose.

$$
\begin{aligned}
& \text { SAMPLE ITEM B } \\
& { }^{6} \text { drum } \\
& { }^{7} \text { having } \\
& { }^{8} \text { qwick } \\
& { }^{9} \text { sure } \\
& { }^{0} \text { none of these }
\end{aligned}
$$

You should have marked answer space 8 for Sample Item B because "qwick" is the misspelled word. 
Do Items 1-32 below and mark your answers on the answer sheet.

Be careful to keep your place on the answer sheet.

$$
\begin{array}{rll}
1 \quad & 1 & \text { chane } \\
& { }_{2} \text { dozen } \\
& { }^{3} \text { size } \\
& 4 & \text { sorry } \\
& 5 & \text { none of these }
\end{array}
$$

$2{ }^{6}$ raise

7 throat

8 whole

9 yuong

${ }^{0}$ none of these

31 crowd

2 inside

3 smile

4 warm

${ }^{5}$ none of these

$4{ }^{6}$ arithmetic

${ }^{7}$ level

8 ridje

9 threading

${ }^{0}$ none of these

$5 \quad$ gather

2 pine

3 somewhere

4 woman

${ }^{5}$ none of these
$6{ }^{6}$ cities

7 dooty

8 human

9 rapid

${ }^{0}$ none of these

$7 \quad 1$ action

2 patch

3 repeat

4 sparow

5 none of these

$8{ }^{6}$ blossom

7 catch

8 door

${ }^{9}$ selery

${ }^{0}$ none of these

91 pasture

2 stalk

$\cdot 3$ waddle

4 zone

${ }^{5}$ none of these

10

${ }^{6}$ flake
${ }^{7}$ hoze
${ }^{8}$ punch
${ }^{9}$ represent
${ }^{0}$ none of these

$11^{1}$ buckle

${ }^{2}$ central

3 husky

${ }^{4}$ precious

5 none of these

12 6 belt

7 inch

8 just

9 samon

0 none of these

131 autum

2 barley

${ }^{3}$ rugged

4 shield

5 none of these

$14{ }^{6}$ napkin

7 quiver

8 tasteing

9 watermelon

${ }^{0}$ none of these

15

$\begin{array}{ll}1 & \text { fade } \\ 2 & \text { lamp } \\ & \text { panther } \\ & \\ & \text { welth } \\ { }^{4} & \text { none of these }\end{array}$




$\begin{array}{rll}16 \quad{ }^{6} & \text { directly } \\ & { }^{7} \text { marvelus } \\ { }^{8} & \text { quarrel } \\ & 9 & \text { witness } \\ & & \text { none of these }\end{array}$

17. ${ }^{1}$ germ

2 hesitate

3 jollyer

4 lane

5 none of these

$18{ }^{6}$ flameing

7 gauze

8 shelter

9 tomatoes

${ }^{0}$ none of these

$19{ }^{1}$, allready

2 breathe

3 chalk

4 lime

5 none of these

206 admire

7 fauset

8 freckle

9 onion.

${ }^{0}$ none of these

$21{ }^{1}$ fern

2 generation

3 gitar

4 harsh

${ }^{5}$ none of these

$$
\begin{array}{rll}
22 & { }^{6} & \text { bowl } \\
& { }^{7} & \text { plaster } \\
& { }^{8} & \text { skarf } \\
& 9 & \text { window } \\
& { }^{0} & \text { none of these }
\end{array}
$$

231 eleven

2 information

${ }^{3}$ knight

4 whitle

${ }^{5}$ none of these

$24{ }^{6}$ dock

7 lawn

8 manafacture

9 zero

${ }^{0}$ none of these

$25{ }^{1}$ comand

2 period

${ }^{3}$ restful

4 wiggling

5 none of these.

$26{ }^{6}$ aquarium

7 banjo

8 miracle

9 shimmering

${ }^{0}$ none of these

27 hut

2 imitate

3 jewlry

4 stove

${ }^{5}$ none of these
$28{ }^{6}$ dust

7 elbow

8 gready

9 hull

${ }^{0}$ none of these

29 giraffe

${ }^{2}$ harnes

${ }^{3}$ hatch

${ }^{4}$ number

${ }^{5}$ none of these

30

6 banana

7 loyal

${ }^{8}$ mineral

9 releif

${ }^{0}$ none of these

$31{ }^{1}$ chatter

2 hawl

3 oats

4 uneasy

5 none of these

$32{ }^{6}$ brush

7 butterfiy

${ }^{8}$ coller

9 question

${ }^{0}$ none of these 



\section{ompu=}

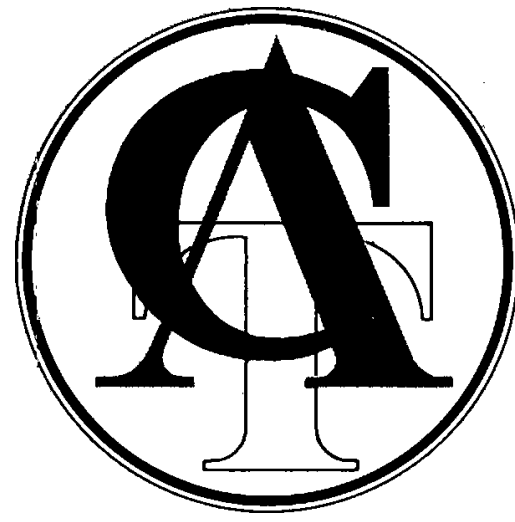

CALIFORNIA ACHIEVEMENT TESTS 1970 Eorrion

\section{Levels $3,4,5$}

Devised by ERNEST W. TIEGS AND WHLLIS W. CLARK

The California Achievement Tests, 1970 Edition is a revision of the well-known achievement series in use for more than 30 years. One of the innovations of this revision is the production of this Compuscan form which allows the examinee to record his answers to items on the entire batter: on a single answer sheet. Heretofore, a minimum of three separate answer sheets had been required for batiery testing.
7 Published by CTB/McGraw-Hill, Del Monte Research Park, Montexey. California 93940. Copyright (C1970 by McGraw-Hill, Inc. All Rights Reserved. Printed in the U.S.A.

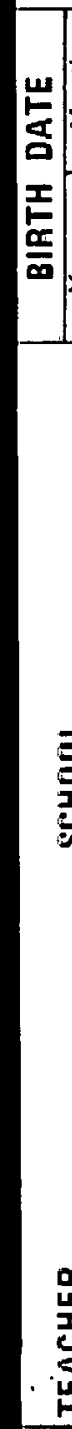

\begin{tabular}{|c|c|c|c|}
\hline 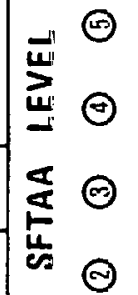 & $\frac{1}{b}$ & $\begin{array}{l}\overline{0} \\
\frac{5}{0} \\
\theta \\
\theta\end{array}$ & 豆 \\
\hline
\end{tabular}

œ̊

(4)

(2)

-

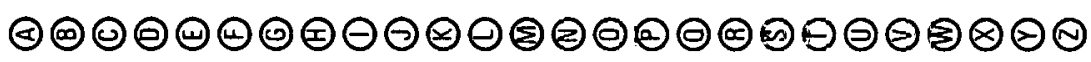

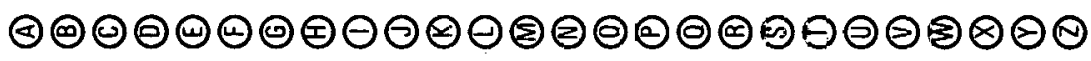

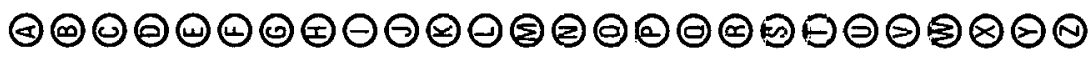

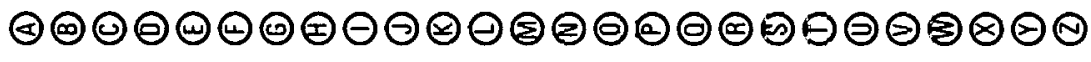

突

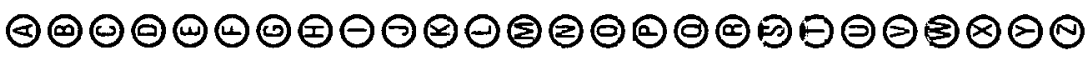

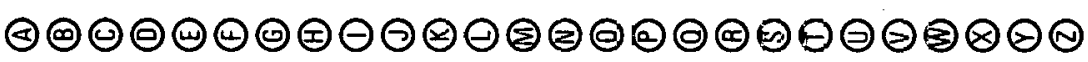

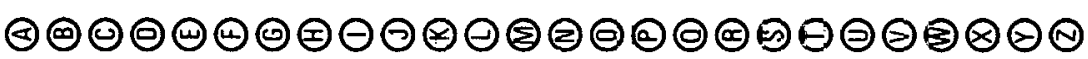

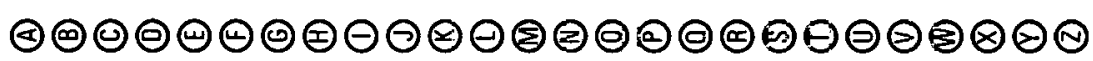

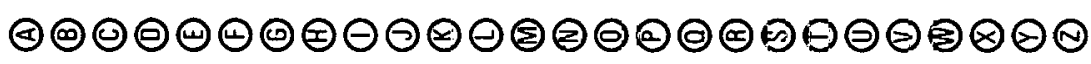

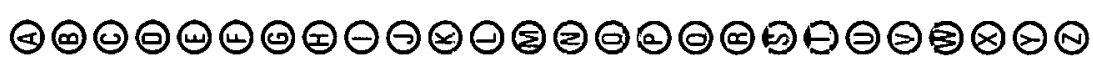

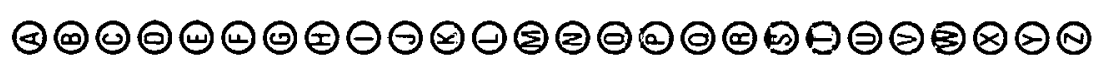

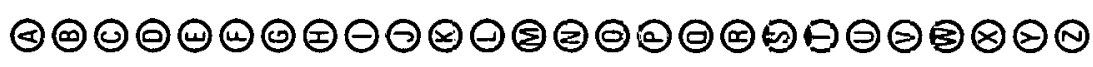

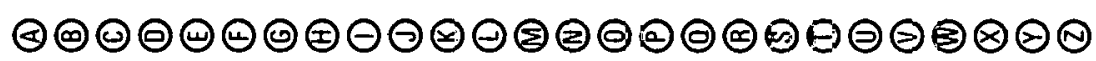

$\Sigma \odot \Theta \Theta \Theta \Theta \Theta(\theta) \oplus$

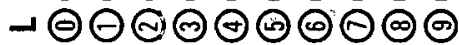

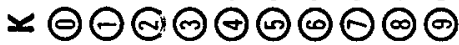

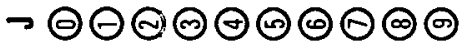

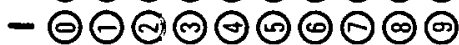

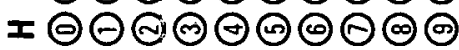

• น ш -

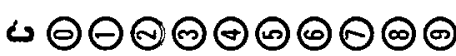

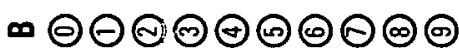

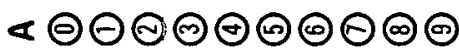
I $\Theta \Theta \odot \Theta \Theta \Theta \Theta \Theta \Theta$ • $\sum_{0}^{2}$

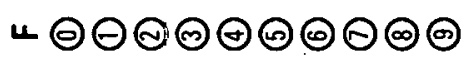
山 ๑ •

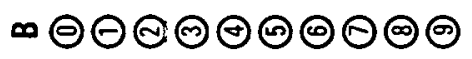

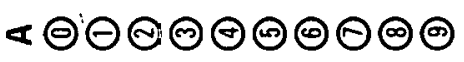




\begin{tabular}{|c|c|c|c|c|c|c|}
\hline $\begin{array}{l}A \text { (1) (2) (3) } \\
B \text { (6) (7) (8) (9) } \\
1 \text { (1) (2) (3) (4) } \\
2 \text { (6) (7) (8) (9) } \\
3 \text { (1) (2) (3) (4) } \\
4 \text { (6) (7) (8) (9) } \\
5 \text { (1) (2) (3) (4) } \\
6 \text { (6) (7) (8) (9) } \\
7 \text { (1) (2) (3) (4) } \\
8 \text { (6) (7) (8) (9) } \\
9 \text { (1) (2) (3) (4) } \\
10 \text { (6) (7) (8) (9) }\end{array}$ & 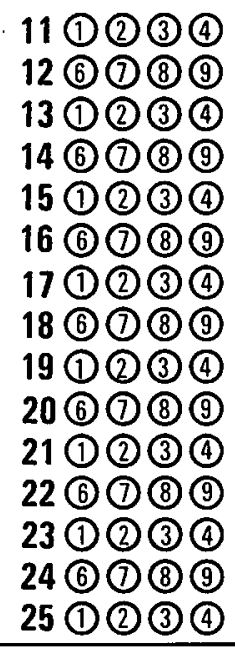 & $\begin{array}{l}26 \text { (6) (1) (8) (9) } \\
21 \text { (1) (2) (3) (4) } \\
28 \text { (6) (7) (8) (9) } \\
29 \text { (1) (2) (3) (4) } \\
30 \text { (6) (1) (8) (9) } \\
31 \text { (1) (2) (3) (4) } \\
32 \text { (6) (7) (8) (9) } \\
33 \text { (1) (2) (3) (4) } \\
34 \text { (6) (1) (8) (9) } \\
35 \text { (1) (2) (3) (4) } \\
36 \text { (6) (1) (8) (9) } \\
37 \text { (1) (2) (3) (4) } \\
38 \text { (6) (7) (8) (9) } \\
39 \text { (1) (2) (3) (4) } \\
40 \text { (6) (1) (8) (9) }\end{array}$ & 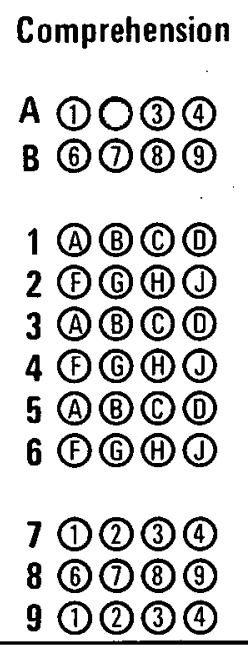 & $\begin{array}{l}15 \text { (1) (2) (3) (4) } \\
16 \text { (6) (7) (8) (9) } \\
17 \text { (1) (2) (3) (4) } \\
18 \text { (6) (7) (8) (9) } \\
19 \text { (1) (2) (3) (4) }\end{array}$ & $\begin{array}{l}28 \text { (6) (1) (8) (9) } \\
29 \text { (1) (2) (3) (4) } \\
30 \text { (6) (7) (8) (9) } \\
31 \text { (1) (2) (3) (4) } \\
32 \text { (6) (7) (8) (9) }\end{array}$ & $\begin{array}{l}36 \text { (6) (7) (8) (9) (1) } \\
37 \text { (1) (2) (3) (4) (5) } \\
38 \text { (6) (7) (8) (9) (1) } \\
39 \text { (1) (2) (3) (4) (5) } \\
41 \text { (6) (7) (8) (9) (1) }\end{array}$ \\
\hline
\end{tabular}

MATHEMATICS

computation $2 \oplus \mathbb{2}(\mathbb{A})(1)$ 3 (A) (B) (C) (D)

$A(A)(B)(C) O$ $B$ () (G) (1) (1) 1 (A) (B) (C) (D) (C)
7 (A) (B) (C) (D) (E) 8 (ㄷ) (1) (1) 9 (A) (B) (C) (D) () 10 ()(B)(1) (1) 11 (A) (B) (C) (D) (C)

12 ()(6)(1)(1) (1) 13 (A) (B) (C) (D) (E) 14 () (G) (1) (1) 15 (A) (B) (C) (D) (C)
$16(\mathbb{A}(\mathbb{A})(1)$ 17 (A) (B) (C) (D) 18 () () (1) (1) 19 (A) (B) (C) (1) (E) 20 ()(B)(1) (1)

21 (A) (B) (C) (1) () 22 ()(ङ)(1)(1) 23 (A) (B) (C) (1) (E) 24 ()(B)(1) (1)
25 (A) (B) (C) (D) () 26 () $(\mathbb{A}(1) \mathbb{B}$ 27 (A) (B) (C) (D) (E) 28 ()ㅕ() (1) (1) 29 (A) (B) (C) (1) (E)

30 ๑() (1) (1) 31 (A) (B) (C) (D) (C) 32 ()( $(1)(1)$ 33 (A) (B) (C) (1) (C)
34 ()ㅗ(1)(1) (1) 35 (A) (B) (C) (1) (E) 36 ()ㅡ(A) (1) 37 (A) (B) (C) (D) (E) 38 () () (1) (1)

39 (A) (B) (C) (D) () 40 () (1) (1) (1) 41 (A) (B) (C) (1) () 42 (F) (6) (1) (1)
43 (A) (B) (C) 44 () $\mathbb{A}$ 45 (A) (B) (C) 46 ()ㅛ (1) 47 (A) (B) (C)

$48 \oplus(\mathrm{G})$

Concepts

5 ()ㅛ(1)®

12 (A) (B) (C) (D) (C)

19 (A) (B) (C) (D) (E)

$26 \oplus \mathbb{1}(1)(1)$

33 (A) (B) (C) (D) (E)

$A$ (A) (B) O(D) 6 (A) (B) (C) (1) (B) 13 (1) (2) (3) (4) (5)

20 (1) (2) (3) (4) (5)

27 (A) (B) (C) (D) (E)

34 ()(요(1) (1)

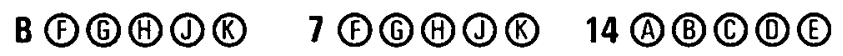

21 (A) (B) (C) (D) (B)

$28(\odot)(1)(1)(1)$

35 (A) (B) (C) (D) ()

1 (1) (2) (3) (4) (5) 8 (A) (B) (C) (1) () 15 (1) (2) (3) (4) (5)

22 () () (1) (1) (B) 29 (A) (B) (C) (D) ()

2 (A) (B) (C) (1) (C) 9 (1) (2) (3) (4) (5) 16 (A) (B) (C) (D) ()

23 (A) (B) (C) (D) (E) 30 (ค) (G) (1) (1)

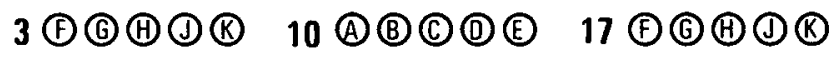

24 () (G) (1) (1) 31 (A) (B) (C) (D) ()

4 (A) (B) (C) (1) () 11 (1) (2) (3) (4) (5) 18 (1) (2) (3) (4) (5)

25 (A) (B) (C) (1) (E) 32 () () (1) (1)

Problems

1 (A) (B) (C) (D) (E)

$4 \oplus(1)(1)$

7 (A) (B) (C) (D) (E)

$10 \oplus \mathbb{1}(\mathbb{A}(1) \mathbb{(}$

13 (A) (B) (C) (D) (C)

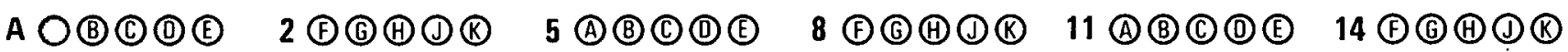

B (도)(1) (B) 3 (A) B(C) (D) (B)

6 ()(1) (1)(1)

9 (A) (B) (C) (D) (C)

$12 \oplus(\mathbb{B})(1)(\mathbb{1}$

15 (A) (B) (C) (D) (E)

Fractions

3 (A) (B) (C) (D) (E)

8 토()(1)

13 (A) (B) (C) (D) (E)

18 ()ㅗㅇㅛ (1) (1)

$A$ (A) (B) (C) $O$ (E)

4 () (1)(1)

9 (A) (B) (C) (D) (E)

14 ()ㅠ⑴ (1)

19 (A) (B) (C) (D) (E)

B () (G) (1) (1)

5 (A) (B) (ㄷ) (1) ()

10 ()ㅛ(1) (1)

15 (A) (B) (C) (D) (C)

$20 \oplus(A)(1)$

1 (A) (B) (C) (D) (E)

6 ()ㅗ()(1)

11 (A) (B) (C) (D) (E)

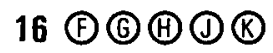

2 (도(1)(1) (1)

12 ()ㅗ (1) (1)

17 (A) B(C) (D) (E) 


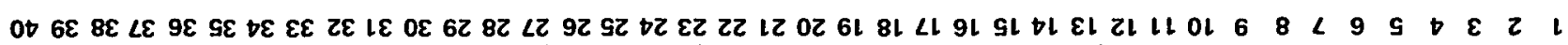

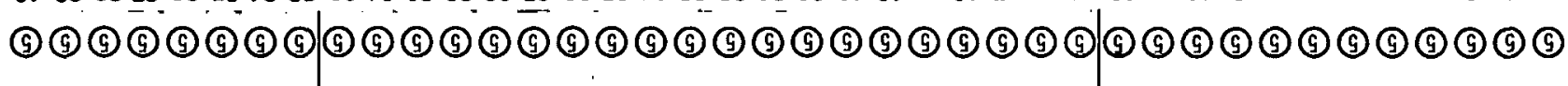

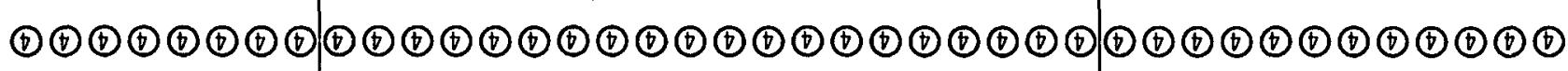

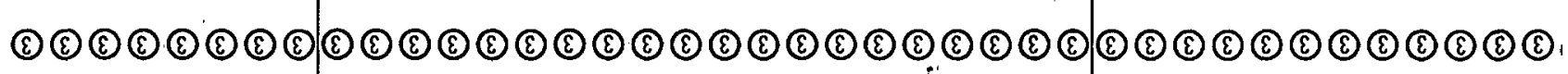

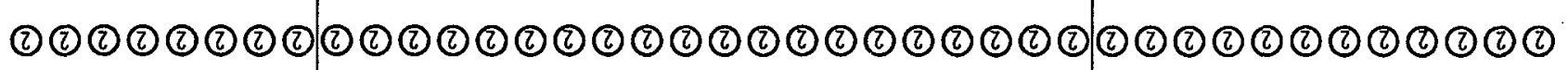

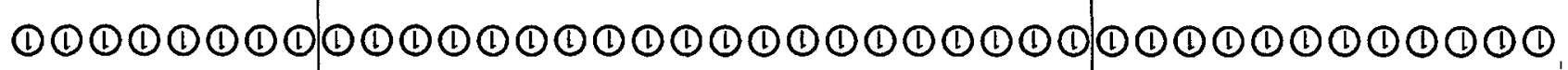

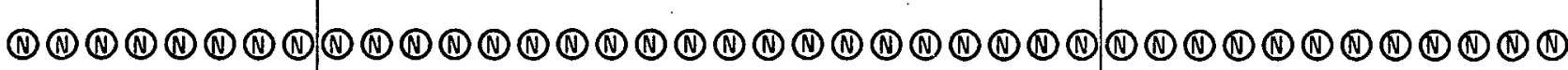
(1) (1) (1) (1) (1) (1) (1) (1) (1) (1) (1) (1) (1) (1) (1) (1) (1) (1) (1) (1) (1) (1) (1) (1) (1) (1) (1) (1) (1) (1) (1) (1)

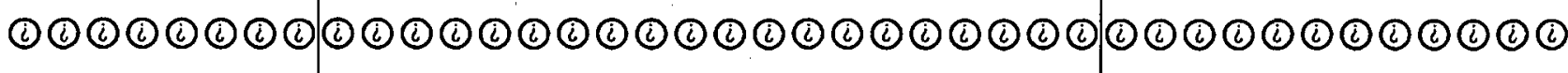

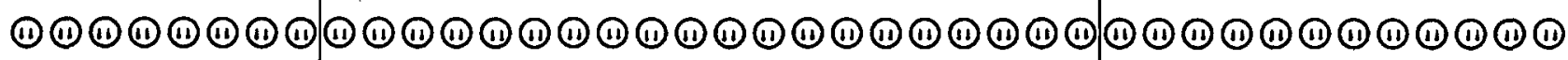

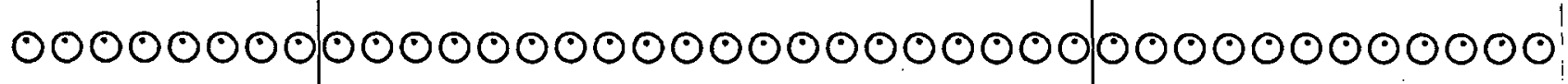

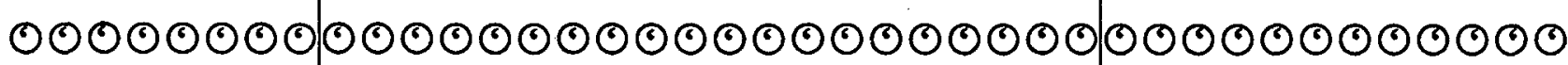

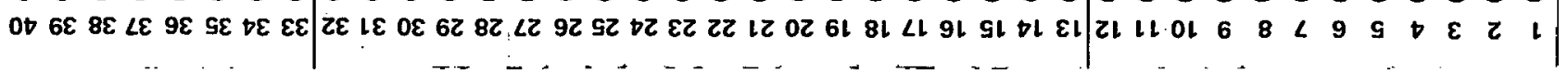

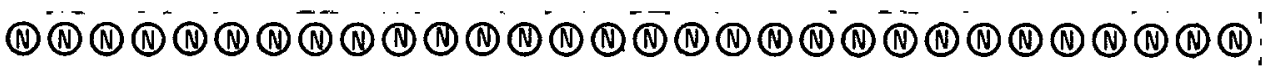

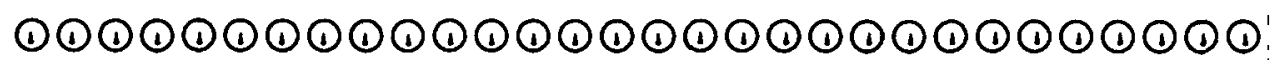

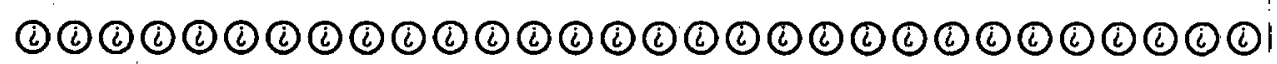

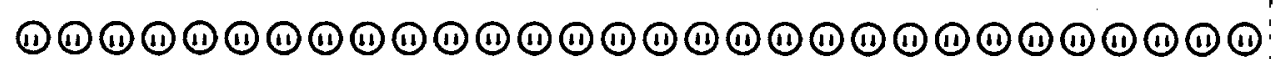

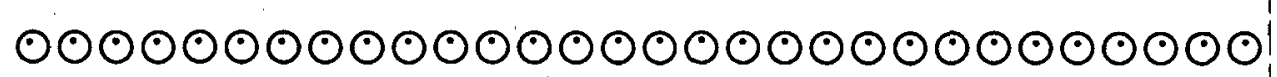

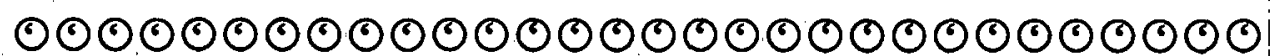

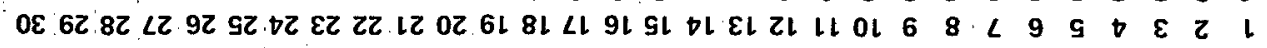
$\ldots \ldots \ldots \ldots \ldots \ldots$

(1)(2)(1)

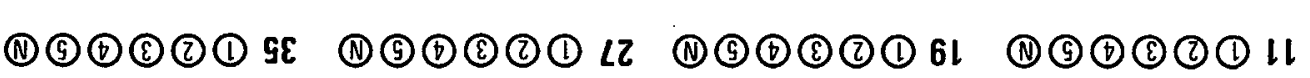

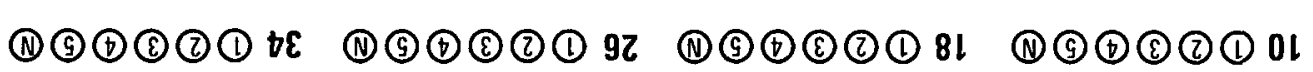

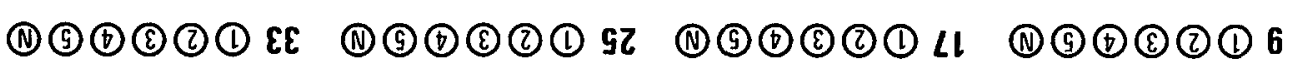

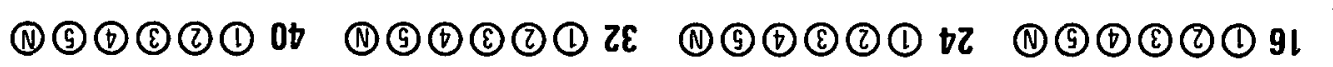
(11)(9)(1)(8)(7) $6 \varepsilon$ (11)(9)(7)(8)(2) (1) $8 E$ (1) (9) (7) (2) (2) (1) $I \varepsilon$.

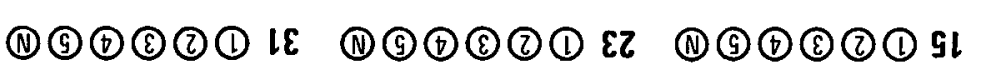

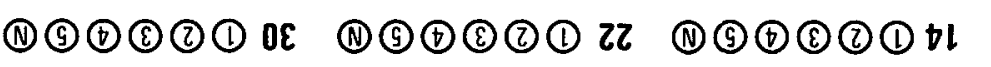

(1) (5) (1) (ㅇ) (2) (1) 62
(II) (9) (1) (2) (2) (1) $\iota 2$

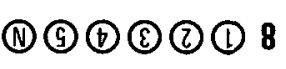

(10)(3)(ㄱ)(2)(1) $L$ (11)(5)(1)(2)(2) (1) 9 (11) (5) (1) (2) (2) (1) 9 a $\forall$

(9) (9) 9 p $10 \mathrm{M}$ (B)(-) $\rightarrow \mathrm{proM}$ (ㄷ) (6) $\varepsilon$ p. (1) (2) 2 P $10 \mathrm{M}$ (1) 1 prom

(11) (11) auoN

(1) (1) ау dostsod (2) (4) Xม⿱

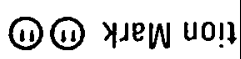
$\mathcal{\odot} \odot$ polad OO emmos a $\forall$

II 40!ฺอกฺว

(11) (11) aUON

(1) (1) aydoussod (1) (3) YมeW (10)(10) Yมew uo!n $\odot \odot$ po!nad

() a $\forall$

I นo!fenเงu (11)(9) (1)(3)(2) (11)(9) (1) (2) (2) (1) (11)(3) (1) (2) (3) (1) (1) (4) (1) (3) (2) (1) (11)(5) (1) (C) (2) (1) (1) (5) (1) uo!fez!||eয!c 
LANGUAGE

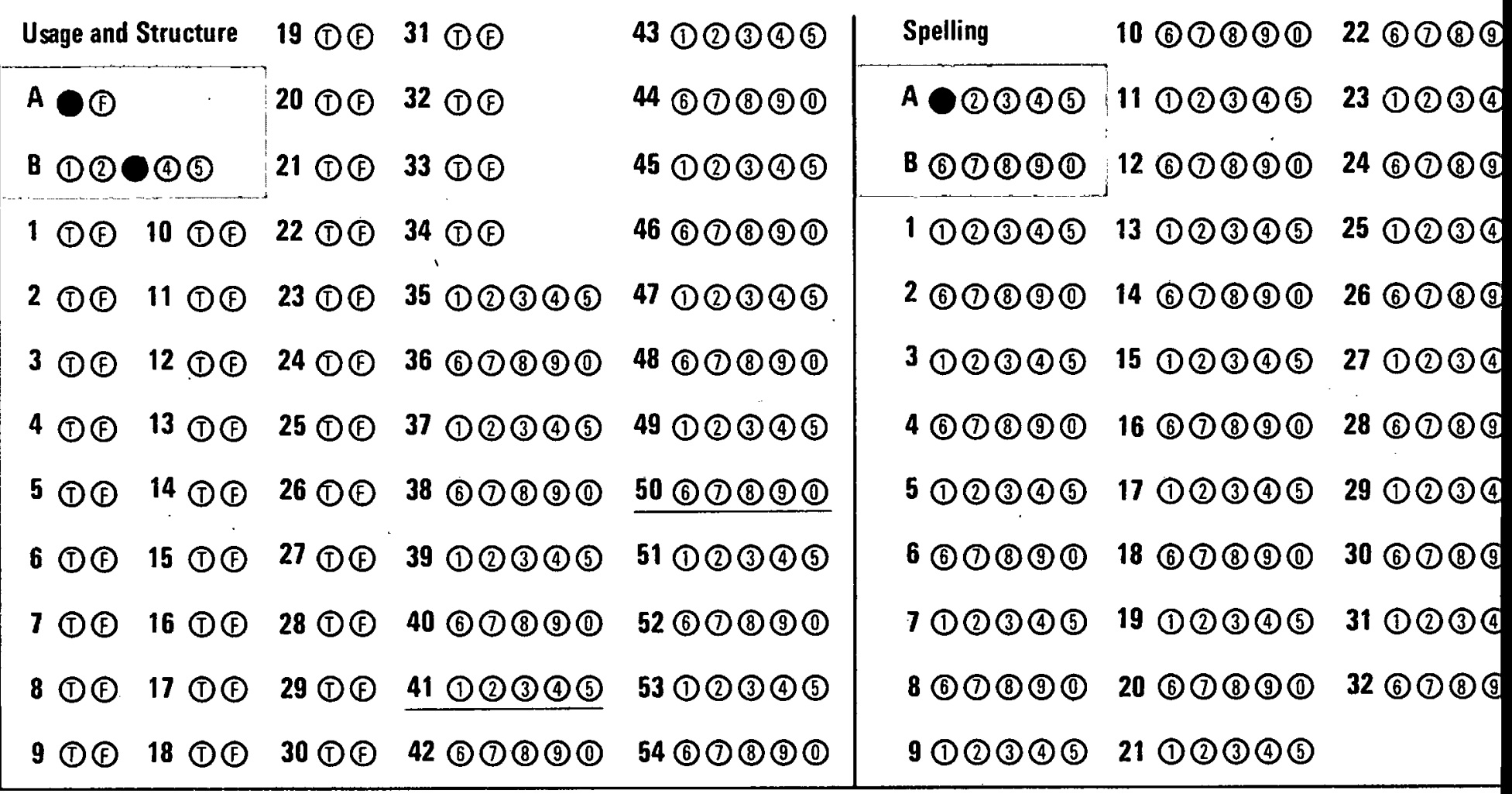


APPENDIX B

CALIFORNIA PERSONALITY TEST 


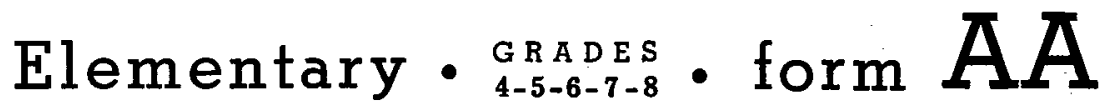

\section{California Test of Personality}

1953 Revision

\section{Devised by}

\section{LOUIS P. THORPE, WILLIS W. CLARK, AND ERNEST W. TIEGS}

Do not write or mark on this booklet unless told to do so by the examiner.

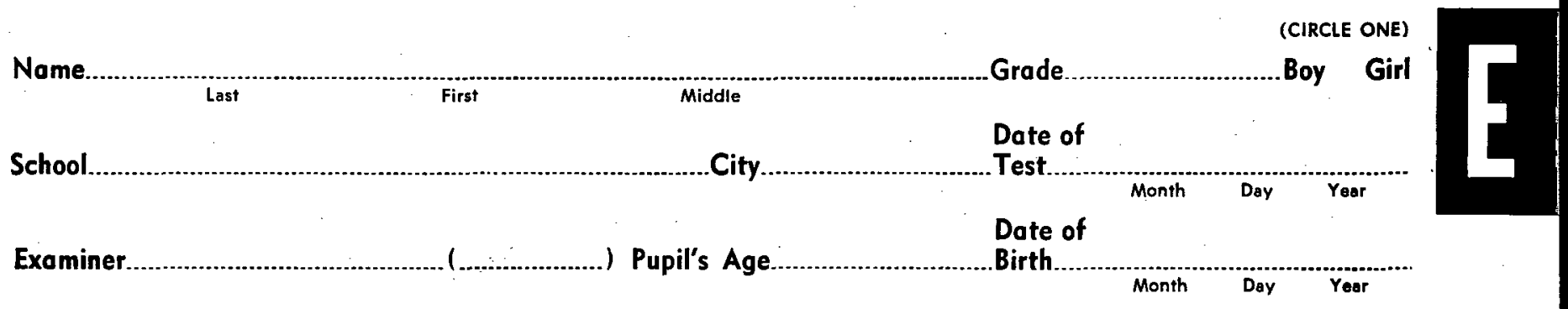

\section{INSTRUCTIONS TO PUPILS:}

This booklet contains some questions which can be answered YES or NO. Your answers will show what you usually think, how you usually feel, or what you usually do about things. Work as fast as you can without making mistakes.

DO NOT TURN THIS PAGE UNTIL TOLD TO DO SO. 


\section{INSTRUCTIONS TO PUPILS}

\section{DO NOT WRITE OR MARK ON THIS TEST BOOKLET UNLESS TOLD TO DO SO BY THE EXAMINER.}

You are to decide for each question whether the answer is YES or NO and mark it as you are told. The following are two sample questions:

\section{SAMPLES}
A. Do you have a dog at home?
YES NO
B. Can you ride a bicycle?
YES NO

\section{DIRECTIONS FOR MARKING ANSWERS}

\section{ON ANSWER SHEETS}

Make a heavy black mark under the word YES or NO to show your answer. If you have a dog at home, you would mark under the YES for question $A$ as shown below. If you cannot ride a bicycle, you would mark under the NO for question $B$ as shown below.

$$
\begin{array}{lll}
\mathrm{A} & \text { YES } \\
\mathrm{B} & &
\end{array}
$$

Remember, you mark under the word that shows your answer. Now find Samples $A$ and $B$ on your answer sheet and show your answer for each by marking YES or NO. Do it now. Find answer row number 1 on your answer sheet. Now wait until the examiner tells you to begin.

\section{ON TEST BOOKLETS}

Draw a circle around the word YES or NO, whichever shows your answer. If you hove a dog at home, draw a circle around the word YES in Sample A above; if not, draw a circle around the word NO. Do it now.

If you can ride a bicycle, draw a circle around the word YES in Sample B above; if not, drow a circle around the word NO. Do it now.

Now wait until the examiner tells you to begin.

After the examiner tells you to begin, go right on from one page to another until you have finished the test or are told to stop. Work as fast as you can without making mistakes. Now look at item 1 on page 3. Ready, begin. 
1. Do you usually keep at your work until it is done?

2. Do you usually apologize when you are wrong?

YES NO

3. Do you help other boys and girls have a good time at parties?

YES NO

4. Do you usually believe what other boys or girls tell you?

YES NO

5. Is it easy for you to recite or talk in class?

YES NO

6. When you have some free time, do you usually ask your parents or teacher what to do?

YES NO

7. Do you usually go to bed on time, even when you wish to stay up?

YES NO

8. Is it hard to do your work when someone blames you for something?

YES NO

9. Can you often get boys and girls to do what you want them to?

YES NO

10. Do your parents or teachers usually need to tell you to do your work?

YES NO

11. If you are a boy, do you talk to new girls? If you are a girl, do you talk to new boys?

YES NO

12. Would you rather plan your own work than to have someone else plan it for you?

YES NO
13. Do your friends generally think that your ideas are good?

YES NO

14. Do people often do nice things for you?

YES : NO

15. Do you wish that your father (or mother) had a better job?

YES NO

16. Are your friends and classmates usually interested in the things you do?

YES NO

17. Do your classmates seem to think that you are not a good friend?

YES NO

18. Do your friends and classmates often want to help you?

YES NO

19. Are you sometimes cheated when you trade things?

YES NO

20. Do your classmates and friends usually feel that they know more than you do?

YES NO

21. Do your folks seem to think that you are doing well?

YES NO

22. Can you do most of the things you try?

YES NO

23. Do people often think that you cannot do things very well?

YES NO

24. Do most of your friends and classmates think you are bright? YES NO 
25. Do you feel that your folks boss you too much?

YES NO

26. Are you allowed enough time to play?

YES NO

27. May you usually bring your friends home when you want to?

28. Do others usually decide to which parties you may go?

YES NO

29. May you usually do what you want to during your spare time?

YES NO

30. Are you prevented from doing most of the things you want to?

YES NO

31. Do your folks often stop you from going around with your friends? YES NO

32. Do you have a chance to see many new things?

YES NO

33. Are you given some spending money?

YES NO

34. Do your folks stop you from taking short walks with your friends?

YES NO

35. Are you punished for lots of little things?

YES NO

36. Do some people try to rule you so much that you don't like it? YES NO

\section{GO RIGHT ON TO}

Page 4

CTP-E-AA

Section $1 \mathrm{C}$

(number right)
37. Do pets and animals make friends with you easily?

YES NO

38. Are you proud of your school? YES NO

39. Do your classmates think you cannot do well in school?

YES NO

40. Are you as well and strong as most boys and girls?

YES NO

41. Are your cousins, aunts, uncles, or grandparents as nice as those of most of your friends?

YES NO

42. Are the members of your family usually good to you?

YES NO

43. Do you often think that nobody likes you?

YES NO

44. Do you feel that most of your classmates are glad that you are a member of the class?

YES NO

45. Do you have just a few friends? YES NO

46. Do you often wish you had some other parents?

YES NO

47. Is it hard to find friends who will keep your secrets?

YES NO

48. Do the boys and girls usually invite you to their parties?

YES NO 
SECTION I E

49. Have people often been so unfair that you gave up?

YES NO

50. Would you rather stay away from most parties?

YES NO

51. Does it make you shy to have everyone look at you when you enter a room?

YES NO

52. Are you often greatly discouraged about many things that are important to you?

YES NO

53. Do your friends or your work often make you worry?

YES NO

54. Is your work often so hard that you stop trying?

YES NO

55. Are people often so unkind or unfair that it makes you feel bad? YES NO

56. Do your friends or classmates often say or do things that hurt your feelings?

YES NO

57. Do people often try to cheat you or do mean things to you?

YES NO

58. Are you often with people who have so little interest in you that you feel lonesome?

59. Are your studies or your life so dull that you often think about many other things?

YES NO

60. Are people often mean or unfair to you?

YES NO
61. Do you often have dizzy spells? YES NO

62. Do you often have bad dreams? YES NO

63. Do you often bite your fingernails?

YES NO

64. Do you seem to have more headaches than most children?

YES NO

65. Is it hard for you to keep from being restless much of the time?

YES NO

66. Do you often find you are not hungry at meal time?

YES NO

67. Do you catch cold easily?

YES NO

68. Do you often feel tired before noon?

YES NO

69. Do you believe that you have more bad dreams than most of the boys and girls?

YES NO

70. Do you often feel sick to your stomach?

YES NO

71. Do you often have sneezing spells?

YES NO

72. Do your eyes hurt often?

YES NO
GO RIGHT ON to

THE NEXT COLUMN

Page 5

CTP-E-AA

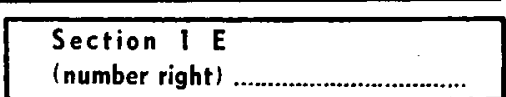

GO RIGHT ON TO THE NEXT PAGE
Section 1

(number right) 
73. Is it all right to cheat in a game when the umpire is not looking?

74. Is it all right to disobey teachers if you think they are not fair to you?

YES NO

75. Should one return things to people who won't return things they borrow?

YES NO

76. Is it all right to take things you need if you have no money?

YES NO

77. Is it necessary to thank those who have helped you?

YES NO

78. Do children need to obey their fathers or mothers even when their friends tell them not to?

79. If a person finds something, does he have a right to keep it or sell it?

YES NO

80. Do boys and girls need to do what their teachers say is right?

81. Should boys and girls ask their parents for permission to do things?

YES NO

82. Should children be nice to people they don't like?

YES NO

83. Is it all right for children to cry or whine when their parents keep them home from a show?

YES NO

84. When people get sick or are in trouble, is it usually their own fault?

YES NO
85. Do you let people know you are right no matter what they say?

YES NO

86. Do you try games at parties even if you haven't played them before?

YES NO

87. Do you help new pupils to talk to other children?

YES NO

88. Does it make you feel angry when you lose in games at parties?

YES NO

89. Do you usually help other boys and girls have a good time?

YES NO

90. Is it hard for you to talk to people as soon as you meet them?

YES NO

91. Do you usually act friendly to people you do not like?

YES NO

92. Do you often change your plans in order to help people?

YES NO

93. Do you usually forget the names of people you meet?

YES NO

94. Do the boys and girls seem to think you are nice to them?

YES NO

95. Do you usually keep from showing your temper when you are angry?

YES NO

96. Do you talk to new children at school?

\section{GO NIGHT ON TO \\ THE NEXT COLUMN}

Page 6

CTP-E-AA
Section 2 A

(number right)
Section 2 B

(number right) 
97. Do you like to scare or push smaller boys and girls?

YES NO

98. Have unfair people often said that you made trouble for them? YES NO

99. Do you often make friends or classmates do things they don't want to?

YES NO

100. Is it hard to make people remember how well you can do things?

YES NO

101. Do people often act so mean that you have to be nasty to them?

YES NO

102. Do you often have to make a "fuss" or "act up" to get what you deserve?

YES NO

103. Is anyone at school so mean that you tear, or cut, or break things?

YES NO

104. Are people often so unfair that you lose your temper?

YES NO

105. Is someone at home so mean that you often have to quarrel?

YES NO

106. Do you sometimes need something so much that it is all right to take it?

YES NO

107. Do classmates often quarrel with you?

YES NO

108. Do people often ask you to do such hard or foolish things that you won't do them?

YES NO
109. Do your folks seem to think that you are just as good as they are?

YES NO

110. Do you have a hard time because it seems that your folks hardly ever have enough money? YES NO

111. Are you unhappy because your folks do not care about the things you like?

YES NO

112. When your folks make you mind are they usually nice to you about it?

YES NO

113. Do your folks often claim that you are not as nice to them as you should be?

YES NO

114. Do you like both of your parents about the same?

YES NO

115. Do you feel that your folks fuss at you instead of helping you?

YES NO

116. Do you sometimes feel like running away from home?

YES NO

117. Do you try to keep boys and girls away from your home because it isn't as nice as theirs?

YES NO

118. Does it seem to you that your folks at home often treat you mean?

YES NO

119. Do you feel that no one at home loves you?

YES NO

120. Do you feel that too many people at home try to boss you? YES NO

\begin{tabular}{|l|}
\hline GO RIGHT ON TO \\
THE NEXT COLUMN \\
\hline $\begin{array}{l}\text { Section } 2 \mathrm{C} \\
\text { (number right) }\end{array}$ \\
\hline
\end{tabular}

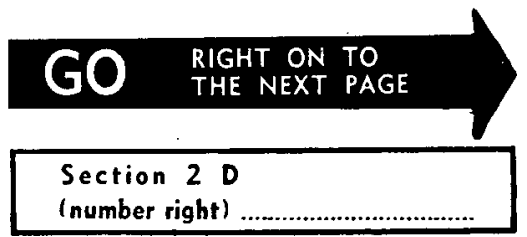

Page 7

CTP-E - AA 
121. Do you think that the boys and girls at school like you as well as they should?

122. Do you think that the children would be happier if the teacher were not so strict?

YES NO

123. Is it fun to do nice things for some of the other boys or girls?

124. Is school work so hard that you are afraid you will fail?

YES NO

125. Do your schoolmates seem to think that you are nice to them?

YES NO

126. Does it seem to you that some of the teachers "have it in for" pupils?

YES NO

127. Do many of the children get along with the teacher much better than you do?

YES NO

128. Would you like to stay home from school a lot if it were right to do so?

YES NO

129. Are most of the boys and girls at school so bad that you try to stay away from them?

YES NO

130. Have you found that some of the teachers do not like to be with the boys and girls?

YES NO

131. Do many of the other boys or girls claim that they play games more fairly than you do?

YES NO

132. Are the boys and girls at school usually nice to you?

YES NO
133. Do you visit many of the interesting places near where you live?

YES NO

134. Do you think there are too few interesting places near your home?

YES NO

135. Do you sometimes do things to make the place in which you live look nicer?

YES NO

136. Do you ever help clean up things near your home?

YES NO

137. Do you take good care of your own pets or help with other people's pets?

YES NO

138. Do you sometimes help other people?

YES NO

139. Do you try to get your friends to obey the laws?

YES NO

140. Do you help children keep away from places where they might get sick?

YES NO

141. Do you dislike many of the people who live near your home?

YES NO

142. Is it all right to do what you please if the police are not around?

YES NO

143. Does it make you glad to see the people living near you get along fine?

YES NO

144. Would you like to have things look better around your home?

YES NO
$G$ RIGHT ON TO

THE NEXT COLUMN

Page 8

CTP-E-AA

Section 2 E

(number right)
STOP NOW WAIT FOR

FURTHER INSTRUCTIONS 


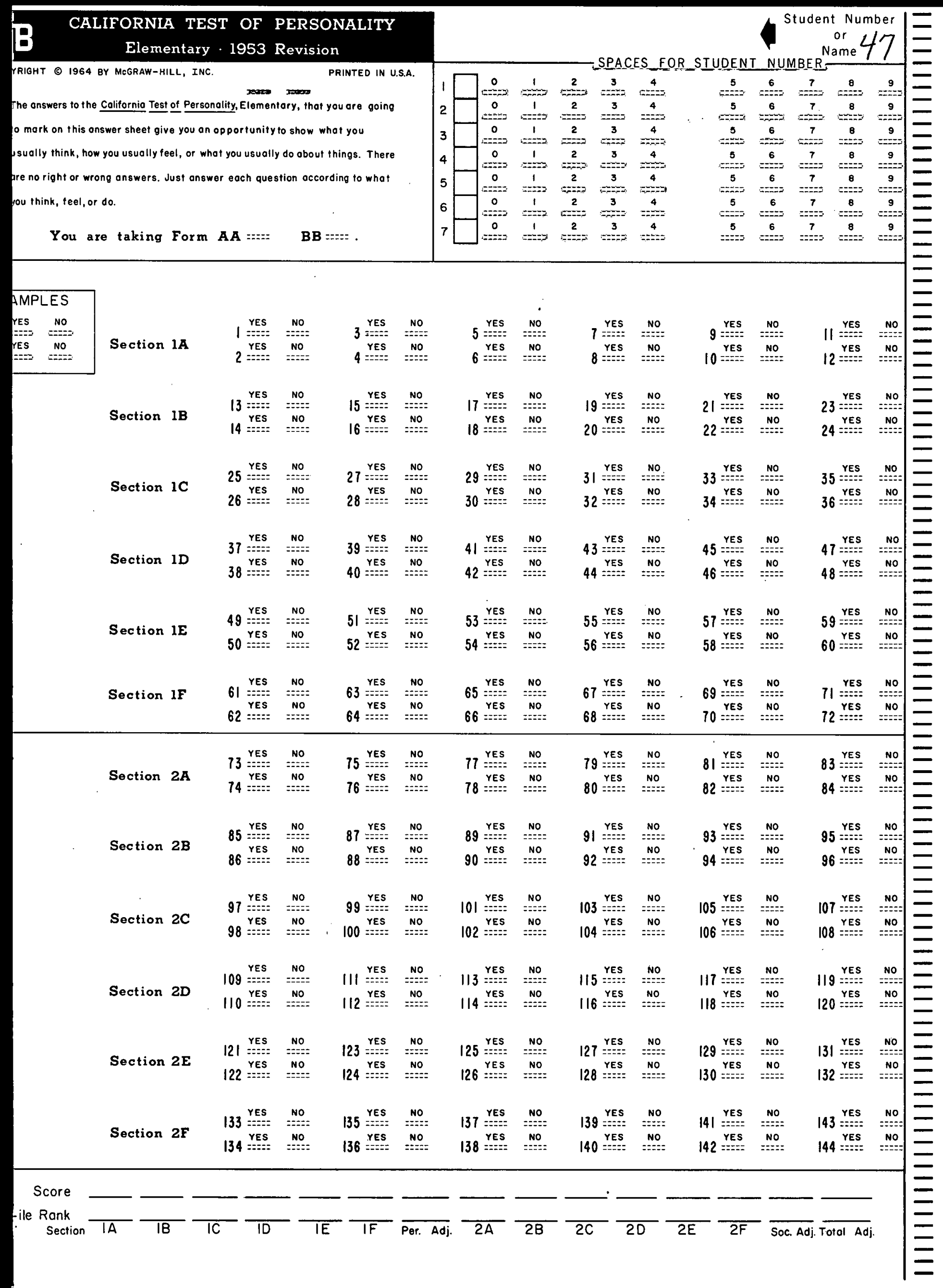


APPENDIX C

MEAN SCORES OF ACADEMIC ACHIEVEMENT

AND SELF-CONCEPT 


\begin{tabular}{|c|c|c|c|}
\hline 砶 & 蛋 & $\underset{x}{\infty}$ & \\
\hline$\stackrel{E}{N}$ & in & Reading & \multirow{4}{*}{ 总 } \\
\hline in & $\stackrel{5}{i}$ & Mathematics & \\
\hline$\stackrel{f}{\infty}$ & i & Language & \\
\hline$\stackrel{f}{\omega}$. & o. & TOTAL BATTERY & \\
\hline$\stackrel{9}{i}$ & is & Self Reliance & \multirow{7}{*}{ 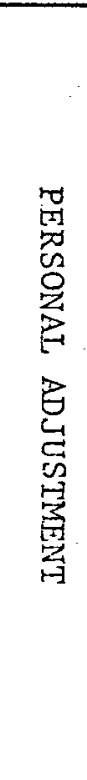 } \\
\hline$\infty_{0}^{\infty}$ & $\stackrel{a}{a}$ & $\begin{array}{c}\text { Sense of Personal } \\
\text { Worth }\end{array}$ & \\
\hline$\dot{0}$ & in & $\begin{array}{c}\text { Sense of Personal } \\
\text { Freedom }\end{array}$ & \\
\hline$\dot{\infty}$ & $\stackrel{v}{2}$ & $\begin{array}{l}\text { Feeling of } \\
\text { Belonging }\end{array}$ & \\
\hline$\stackrel{f}{\infty}$ & $\ddot{v}$ & $\begin{array}{l}\text { Withdrawing } \\
\text { Tendencies }\end{array}$ & \\
\hline in & in & Nervous Symptoms & \\
\hline$\stackrel{f}{i}$ & 5 & $\begin{array}{l}\text { TOTAL PERSONAL } \\
\text { ADJUSTMENT }\end{array}$ & \\
\hline$\stackrel{0}{i}$ & $\dot{\infty}_{\infty}^{\infty}$ & Social Standards & \multirow{8}{*}{ 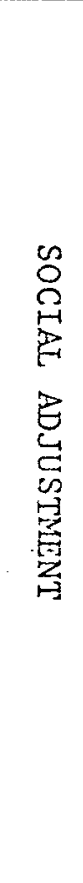 } \\
\hline $\begin{array}{l}\infty \\
0 \\
+\end{array}$ & $\stackrel{2}{\circ}$ & Social Skills & \\
\hline$\dot{\omega}$ & $\infty$ & $\begin{array}{l}\text { Anti-social } \\
\text { Tendencies }\end{array}$ & \\
\hline$\stackrel{\infty}{2}$ & in & Family Relations & \\
\hline$\dot{w}$ & in & School Relations & \\
\hline$\stackrel{b}{i}$ & $\dot{\omega}$ & Community Relations & \\
\hline $\begin{array}{l}\tilde{y} \\
\dot{w}\end{array}$ & $\begin{array}{l}5 \\
\infty \\
\infty\end{array}$ & $\begin{array}{l}\text { TOTAL SOCIAL } \\
\text { ADJUSTMENT }\end{array}$ & \\
\hline$\stackrel{0}{N}$ & $\begin{array}{l}\infty \\
+ \\
-\end{array}$ & $\begin{array}{c}\text { TOTAL } \\
\text { SELF-CONCEPT }\end{array}$ & \\
\hline
\end{tabular}


BIBLIOGRAPHY

Books

Bernard, Harold W. Human Development in Western Culture. Boston: Allyn and Bacon, Inc., 1962.

Brookover, W., and Lapere, J. Self-Concepts of Ability and School. Achievement in Junior High School Subjects. Michigan State University, 1962.

Buhler, Kar1. The Hental Development of the Child. New York: International Ljterature of Psychology, Philosophy and Science, 1954.

Buros, Oscar Krisen. The Sixth Mental Measurement Yearbook. Highland Park, New Jersey: The Cryphon Press, 1965.

Charles, Don C. Psychology of Child in the Classroom. New York: The Macmillan, 1964.

Combs, Arthur and Saỹón, Donald. Individual Behavior. New York: Harper and Row, 1959.

Gale, Raymond F. Developmental Behavior. London: The Macmillan Com-pany, 1969 .

Gordon, Ira J. Human Development From Birth Through Adolescence. 2nd ed. New York: Harper \& Row, Publishers, 1969.

Ferguson, George A. Statistical Analysis in Psychology \& Education. 3rd ed. New York: McGraw-Hill Book Company, 1971.

Fisher, Ronald A. and Frark Yates. Statistical Tables for Biological, Agricultural, and Medical Research. 6th ed. New York: Hafner Publishing Co., 1963 .

Jersild, Arthur T. Child Psychology. 4th ed. New York: Macmillan Co., 1954. 1952

In Search of Self. New York: Columbia University Press,

Kelley, EarI c. Another Look at Individualism. Detroit: Wayne State University, 1962.

Kleinmuntz, Benjamin. Personality Measurenent. Homewood, Illinois: The Dorsey Press, 1967. 
Lecky, P. Self Consistency. New York: Island Press, 1951.

Mussen, Henry; Conger, J. Janeway: and Kagan, Jerome. Child Development and Personality: New York: Harper \& Row, Publsihers, 1969 .

Ridenour, Nina. Building Self-confidence in Children. Chicago: Science Research Associates, Inc., 1954.

Rogers, C. R. Client Centered Therapy. New York: Houghton-Mifflin, 1951.

Stephenson, W. The Study of Behavior: Q-Technique and Its Methodology. Chicago: University of Chicago Press, 1953.

Symonds, Percival. The Ego and the Self. New York: Teacher's College, 1952.

Thorpe, Louis P.; Clark Willis W.; and Tiegs, Ernest W. California Test of Personality (Manual). Monterey, California: McGraw-Hill Inc., 1953.

Warner, W. Lloyd. Social Class in America. New York: Harper \& Row, Publishers, 1960.

White, Ellen G. Child Guidance. Nashville: Southern Publishing Association, 1954.

Wylie, R. C. The Self-Concept. Lincoln: University of Nebraska Press, 1961.

\section{$\underline{\text { Periodicals }}$}

Bledsoe, Joseph C. "Self-Concept of Children and Their Intelligence, Achievement, Interest, and Anxiety." Childhood Education, VIIL (March, 1967), 436-438.

Davidson, H. and Jong, G. "Children's Perceptions of the Teacher's Feeling Toward Them Related to Self Perception, School Achievement, and Behavior." Journal of Experimental Education, XXIX (December, 1960), 107-118.

Caplin, Horris D. "Self Concept, Level of Aspiration, and Academic Achievement." The Journal of Negro Education, XXXVII (1968), 435-439.

Day, M. M. "Building Healthy Self-Image." Instructor, LXXIV (January, 1965), 22 . 
Ellsworth, S. G. "Building the Child's Self-Concept." NEA Journa1, LVI (December, 1965), 490-95.

Fink, M. B. "Self Concept as It Relates to Academic Underachievement." California Journal of Educational Research, XIII (1962), 57-62.

Gillham, Isabel. "Self Concept and Reading." The Reading Teacher, XXI (December, 1967), 270-273.

Hawk, Travis L. "Self-Concept of the Socially Disadvantaged." The Elementary School Journal, LXVII (January, 1967), 196-206.

Jacobs, James N. and Felix, Joseph L. "Testing Educational and Psychological Developinent of Preadolescent Children--Ages 6-12." Preview of Educational Research, XXXVIII (December, 1968), 19-28.

Page, E. G. "Teacher Comments and Student Performance: A Seventy-Four Classroom Experiment in School Motivation." Journal of Educational Psychology, XIIX (August, 1958), 173-81.

Paschal, Billy J. "The Role of Self Concept in Achievement." The Journal of Negro Education, XXXVII (1968), 392-396.

Pigge, F. L. "Children and Their Self Concepts." Childhood Education, XLVII (November, 1970), 107-114.

Piers, Ellen V. and Harris D. B. "Age and Other Correlates of the Self Concept in Children." Journal of Educational Psychology, LV (1964), 91-95.

Phillips, Romeo Eldridge. "Student Activities and Self-Concept." The Journal of Negro Education, XXXVIII (1969), 32-37.

Sax, Gilbert. "Concept Formation." Encyclopedia of Education Research. 4 th ed., 1969.

Sears, R. R. "Relation of Early Socialization Experiences to Self Concept and Gender Role in Middle Childhood." Child Development, XLI (January, 1970), 267-289.

Soares, Anthony T. and Soares, Louise M. "Self-Perception of Culturally Disadvantaged Children." American Educational Research Journal, VI (January, 1969), 31-45.

Spaulding, Robert L. "Achievement, Creativity, and Self-Concept Correlates of Teacher-Pupil Transactions in Elementary School Classroom." Cooperative Research Project No. 1352, U.S. Department of Health, Education and Welfare, 1963. 
Sylvester, E. and Kunet, $M$. "Psycho-dynamic Aspects of the Reading Problem." Journal of Orthopsychiatry, XIII (1963), 69-76.

Witty, Paul A. (Ed.) "The Educationally Retarded and Disadvantaged." Sixty-sixth Yearbook, Part I. Chicago, I11.: National Society for the Study of Education, 1967, 384.

Woolner, Roselette B. "Self-Concept--What is it?" Instructor, LXXX (March, 1971), 60.

Zirkel, Perry A. and Moses, E. Gnanaraj. "Self-Concept and Ethnic Group Membership Among Public School Students." American Educational Research Journal, VIII (January, 1971), 253-265.

\section{Unpublished Materials}

Combs, Arthur. "Modern Concepts of Learning." An Address given to the Michigan Department of Education staff, Hay 1966. (mimeographed).

Cummings, Ruby Ne11. Study of the Relationship Between Self Concepts and Reading at Third-Grade Level. Unpublished Ed. Thesis, University of Alabama, August 1970.

Payne, Bill F. and Dunn, Charleta J. Using Group Counseling to Alter Self-Concepts of Culturally Different Elementary Children. Payne: Lamar State College of Technology. Dunn: University of Houston.

Urbish, Millie Mae. Development and Evaluation of Multimedia Instruction on Due Process of Law for Sixth Grade Children. Unpublished Ed. D. Dissertation, University of Maryland, 1970.

Whisenton, Zadie $\mathbb{B}$. The Effects of Planned Experiences With Outstanding Negro Citizens of the Community on the Self-Concept of Negro Children. Unpublished Ph.D. Thesis, University of Alabama, May 1970 . 
